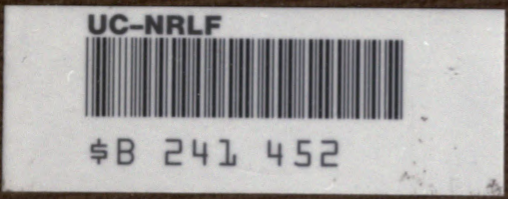



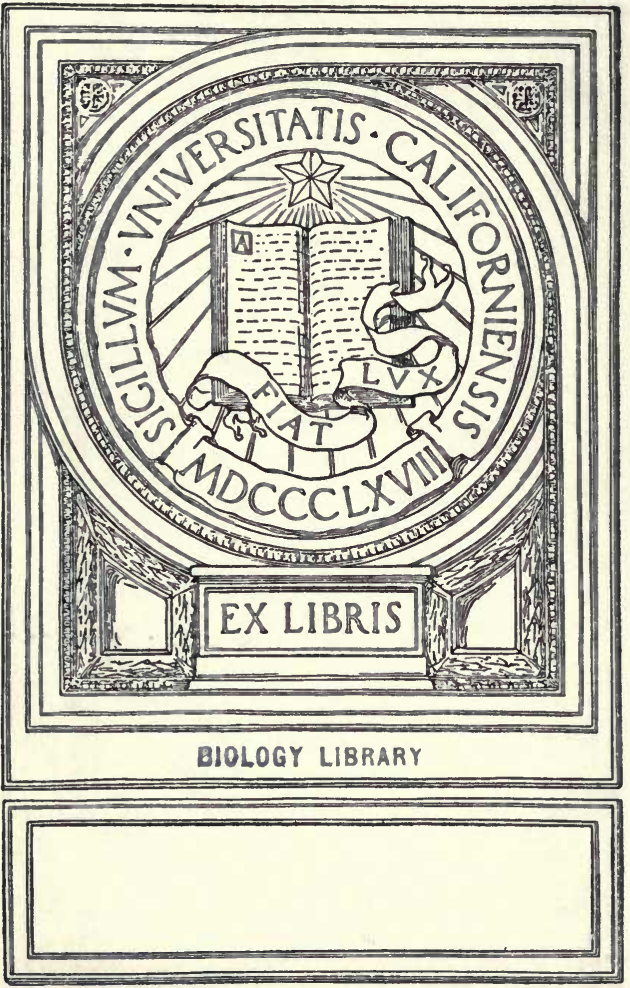



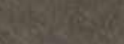

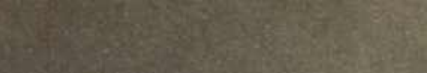

4. (2) 9 sathe $x^{2}$

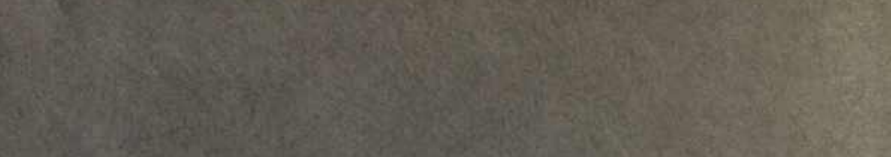
15: 5.4.

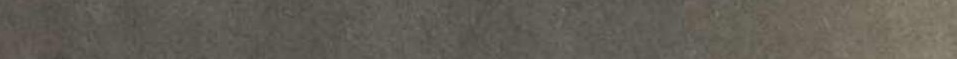

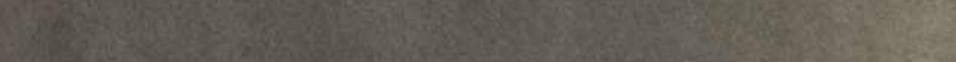

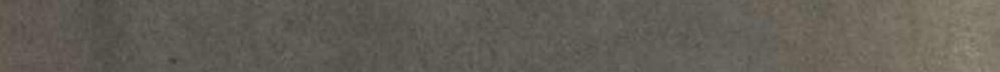

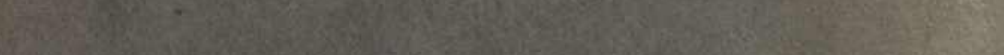

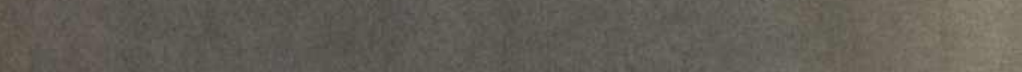

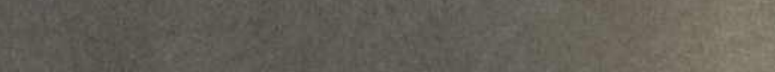
Qu.t.

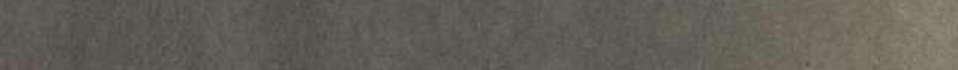
Q8. Whas U. Fon

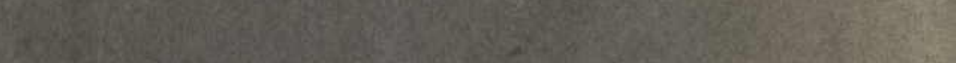

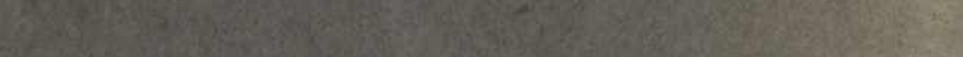
20.5

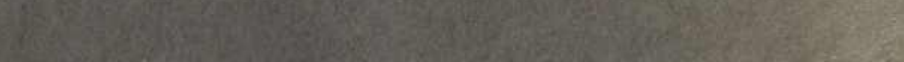

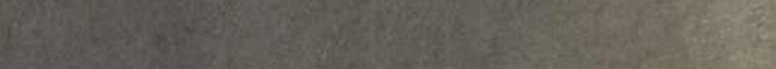

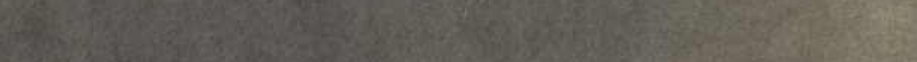
AEx

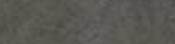

Mes

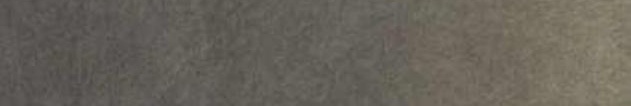
ons

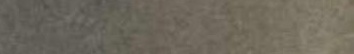
39.

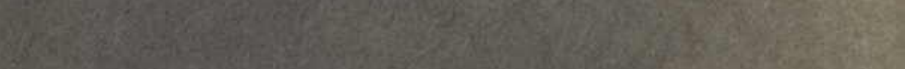

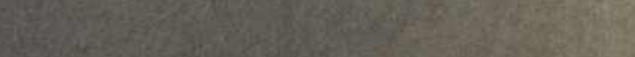
104: for

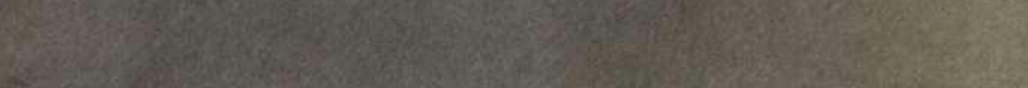
A. 




\section{, EXPERIMENTAL}

\section{DAIRY BACTERIOLOGY •}

BY

H. L. RUSSELL, PH.D.

DEAN OF THE COLLEGE OF AGRICULTURE UNIVERSITY OF WISCONSIN

AND

E. G. HASTINGS, M.S.

ASSISTANT PROFESSOR OF AGRICULTURAL BACTERIOLOGY UNIVERSITY OF WISCONSIN

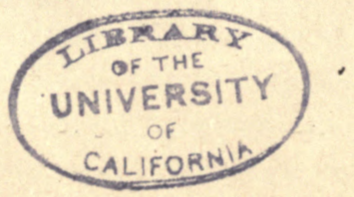

GINN AND COMPANY

BOSTON • NEW YORK - CHICAGO - LONDON 


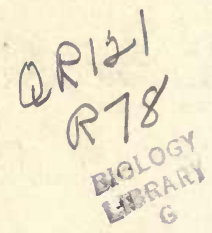

Copyright, 1909, BY

H. L. Russell and E. G. Hastings

ALL RIGHTS RESERVED

99.6

The Atbenaum Press 


\section{INTRODUCTION}

The purpose of the course here outlined is to train the student in those bacteriological processes that are necessary for him to comprehend thoroughly, before he is in a position to appreciate the relation of microörganisms to dairy processes. This work is of fundamental importance to the student who wishes to learn the nature of the biological changes going on in milk and its products, whether he is concerned purely with the practical side of dairying or is interested in the cognate work of dairy chemistry or dairy bacteriology.

The attempt has been made to keep the scope of this work within the realm of dairy bacteriology, and not encroach upon the field of dairy manufactures. For example, in the study of starters it is desirable that the bacteriological student should know how to determine the purity and vigor of a culture, but the practical propagation of the starter should be presented from the creamery point of view. The effect of the ripening of cream on the churning process and the action of acid on the physical condition of cheese are instances of biologic activity that can better be studied in the factory than in the laboratory. In many cases it would be advantageous if the practical and theoretical work could be carried on simultaneously. Thus, in studying a starter bacteriologically it would be very desirable to test in the laboratory by frequent examinations the purity of the starter as it is handled in the dairy from day to day. The possibility of deterioration could then be ascertained from both the practical and scientific points of iii 
view, and each would supplement the other. Such studies, however, are better adapted to the advanced student than to the beginner, and it is felt that it is preferable to present to the elementary student the biologic aspect of the subject more or less completely divorced from the practical factory work. The technique of bacteriological methods should be mastered by each student individually. He should learn how to make his media, and how to care for the same. After this necessary technical foundation has been laid, it is possible to make certain experiments in groups, and thus economize in time and material with no loss in pedagogic value.

The methods presented are believed to be the best in use at the present time. A committee of the American Public Health Association now has under consideration the formulation of standard methods for milk analysis, but these have not as yet been published. The methods of media making are those recommended by the Laboratory Section of the American Public Health Association, and, while more complicated than those usually described in text-books, are surely more desirable in establishing uniform methods.

In bacteriology, as in other biological sciences, unknown factors must be dealt with. Again, numerous conditions that cannot be controlled influence the results. A long series of observations may be certain to point in a definite direction, while one or two observations, such as can be made in a course of study, may give results wholly at variance with what is expected. This fact does not, however, lessen the value of the exercise in training the student in technique and in developing his power of observation. 


\section{CONTENTS}

CHAPTER

Pages

I. Cultural Technique . . . . . . . . . . . . . 1-36

Glassware . . . . . . . . . . . . . . . . . 1

Cleaning glassware . . . . . . . . . . . . . . 2

Sterilization of glassware . . . . . . . . . . . . 3

Preparation of media . . . . . . . . . . . . . 4

Ingredients of media . . . . . . . . . . . . . . 5

Reaction of media . . . . . . . . . . . . . 5

Neutralization of media . . . . . . . . . . . . . 6

Filtering of media . . . . . . . . . . . . . . . . . 7

Plugging tubes . . . . . . . . . . . . . . 9

Sterilization of media . . . . . . . . . . . . . 9

Care of media . . . . . . . . . . . . . . . . . . 11

Preparation of broth . . . . . . . . . . . . . . 12

Preparation of gelatin . . . . . . . . . . . . . 14

Preparation of agar . . . . . . . . . . . . . 15

Preparation of milk . . . . . . . . . . . . . . . . . 16

Preparation of sugar media . . . . . . . . . . . 17

Preparation of litmus solution . . . . . . . . . 18

Preparation of water blanks . . . . . . . . . . . 18

Quantitative analysis of milk . . . . . . . . . 19

Qualitative analysis of milk . . . . . . . . . . . 29

Isolation of pure cultures . . . . . . . . . . . 30

Platinum needles . . . . . . . . . . . . 30

Plate cultures . . . . . . . . . . . . . . . . . 31

Study of plate cultures . . . . . . . . . . . . 32

Test-tube cultures . . . . . . . . . . . . . . 33

Study of test-tube cultures . . . . . . . . . . . 35

II. Microscopical Technique . . . . . . . . . . 37-52

The microscope and its accessories . . . . . . . . . 37

Slides and cover glasses . . . . . . . . . . . . 41

Staining solutions . . . . . . . . . . . . . 42 
Making of stained preparations . . . . . . . . . 44

Gram's stain . . . . . . . . . . . . . 47

Flagella stain . . . . . . . . . . . . . 47

Capsule stain . . . . . . . . . . . . 48

Spore stain . . . . . . . . . . . . . . 48

Examination of living bacteria . . . . . . . . . 49

Hanging block . . . . . . . . . . . . . . 51

Measuring bacteria. . . . . . . . . . . 51

III. Contamination of Milk . . . . . . . . . . 53-63

Contamination from barn air . . . . . . . . . 54

Contamination from coat of animal . . . . . . 56

Contamination from manure . . . . . . . . 57

Contamination from hair . . . . . . . . . . . 58

Contamination from interior of udder . . . . . . 58

Contamination from utensils . . . . . . . . 60

Washing utensils . . . . . . . . . . . . . 63

Plan for the production of sanitary milk . . . . . 63

IV. Milk Fermentations . . . . . . . . . . 64-85

Preliminary cultivation . . . . . . . . . . . 65

Primary tests for identification of species . . . . . . 66

Action on carbohydrates . . . . . . . . . . 67

Action on nitrates . . . . . . . . . . . . . . 70

Formation of indol . . . . . . . . . . . . . 72

Relation to oxygen . . . . . . . . . . . 72

Relation to temperature . . . . . . . . 73

Chromogenesis . . . . . . . . . . . 74

Action on milk . . . . . . . . . . . . . 74

Acid fermentation . . . . . . . . . . . . 76

Digesting and sweet-curdling fermentation . . . . 77

Alcoholic fermentation . . . . . . . . . 78

Ropy fermentation . . . . . . . . . . . . . . 79

Bitter fermentation . . . . . . . . . . . . 80

Cycle of fermentations in milk . . . . . . . . . . 80

Butyric-acid fermentation . . . . . . . . . 81 
V. Preservation of Milk . . . . . . . . . . 86-92

Effect of temperature on bacterial growth . . . . . 88

Pasteurization of milk . . . . . . . . . . . 89

Detection of heated milk. Storch's reaction . . . . . 91

Vi. Relation of Bacteria to Butter . . . . 93-102

Effect of creaming on the distribution of the bacteria in

milk . . . . . . . . . . . . . . . . 93

Examination of cream and separator slime . . . . . 94

Examination of sweet and ripened cream . . . . . . 94

Relation of butter flavor to bacterial development . . . 95

Quantitative analysis of butter . . . . . . . . 95

Relation of age of butter to bacterial content . . . . 96

Starters . . . . . . . . . . . . . 97

Relation of bacteria to keeping quality of butter . . . . 101

Relation of bacteria in wash water to butter . . . . . 101

VII. Relation of Bacteria to Cheese . . . . . 103-109

Ripening of cheese . . . . . . . . . . 103

Rôle of acid-forming bacteria . . . . . . . . 104

Analysis of cheese . . . . . . . . . . . . 105

Qualitative examination of milk for cheese making . . 106

VIII. MILK HYGIENE • • • • • • : • • . • $110-124$

Milk as a distributer of disease . . . . . . . . 110

Microscopical examination for tubercle bacilli . . . . 112

Examination for tubercle bacilli by animal inoculation . 114

Examination for pyogenic organisms . . . . . . . 115

Microscopical examination of milk . . . . . . . . 116

Examination for leucocytes, Doane-Buckley method . . 117

Examination for leucocytes; smeared-sediment method . 120

Examination for fibrin . . . . . . . . . . . 122

Direct enumeration of bacteria in milk . . . . . . . 122

Microscopic examination for streptococci . . . . . 124 
Appendix A. Preparation of Pipettes . . . . . . 125

Appendix B. Descriptive Chart. Society of American BACTERIOLOGISTS . . . . . . . . . . . . 127

Appendix C. Conversion Factors for Thermometer Scales. Conversion Factors. Metric to English. English to Metric . . . . . . . . . . . 138

Appendix D. Apparatus for each Student . . . . 139 Glossary of Terms . . . . . . . . . . . . . . . 140

Text-Books on General and Dairy Bacteriology . . 143 INDEX . . . . . . . . . . . . . . . . . 145 


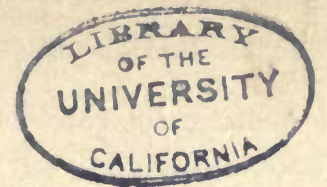 \\ EXPERIMENTAL \\ DAIRY BACTERIOLOGY}

CHAPTER I

\section{CULTURAL TECHNIQUE}

Glassware. In bacteriological work a large amount of glassware must be used. It is desirable to employ the most convenient and economical types of tubes, dishes, etc. The test tubes used for the storage of media for culture work are not the ordinary thin-walled test tubes of the chemical laboratory, but are made of much heavier glass and without the lip. Such tubes of thick glass are not so easily broken in handling and yet will withstand the action of heat. A tube $120 \mathrm{~mm}$. long and $15 \mathrm{~mm}$. internal diameter is a convenient size, and of sufficient capacity for nearly all purposes. If it is desirable to have a tube holding 10 cc., tubes of $18 \mathrm{~mm}$. diameter will be required. The tubes in use in the laboratory should always be of the same length, $120 \mathrm{~mm}$. or $150 \mathrm{~mm}$. The tube described is usually known as the "board of health" pattern.

Petri dishes should have an internal diameter of $90 \mathrm{~mm}$., and should be $15 \mathrm{~mm}$. high; the covers should be $10-12 \mathrm{~mm}$. deep, and should be $2-3 \mathrm{~mm}$. greater in diameter than the bottoms. The bottoms of the Petri dishes should be perfectly flat. The glass from which tubes and dishes are made should 
withstand repeated heating to $170^{\circ} \mathrm{C}$. without flaking or becoming cloudy, and should be well annealed, so as to withstand sudden changes in temperature.

For storage of media, and for making the dilutions necessary in bacteriological work, flasks are used, usually of the Erlenmeyer form, with narrow necks. The flasks may be of Jena glass, but the many forms of " resistance glass" are well

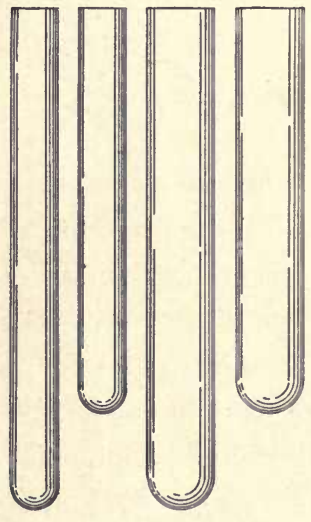

Fig. 1. Test Tubes

Board of health pattern, one-third size adapted to ordinary purposes, since its solubility is slight. For many purposes, as for dilution work, ordinary bottles may be employed; they have the advantage of being cheap and economical of space in sterilizers and elsewhere.

Cleaning glassware. All glassware used in the bacteriological laboratory must be perfectly clean. New glassware must be washed in hot water, rinsed in distilled or tap water, and placed for a short time in 1 per cent hydrochloric acid, to remove any free alkali present on the glass. After rinsing, it is drained and allowed to dry. Petri dishes, tubes, etc., containing discarded cultures, should not be allowed to become dry, but should be washed as soon as possible. The solid media can be readily removed from test tubes by inserting a piece of glass tubing, which is attached to the water tap by a strong piece of rubber tubing. The tube is pushed to the bottom of the test tube and the force of the water ejects the solid matter. Glassware containing whole milk, butter, or any fatty substance should not be washed with the ordinary dishes. If the media has been allowed to become dry in the tubes and plates, they must be 
boiled in water or allowed to stand in a strong solution of washing powder for twenty-four hours. The washing powders which are so largely used in dairy work will be found preferable to soap, rinsing from the glass much easier and producing no deposits of calcium salts with hard water.

Pipettes which have been used for milk should be rinsed at once, placed in a solution of washing powder for several hours, removed, rinsed well, and drained.

The destructive test-tube brush should be replaced by a swab, made by slightly expanding the end of a piece of glass tubing and fastening to this, with an ordinary rubber band, a bit of soft sponge, which may be renewed as often as necessary.

Exercise. Clean all test tubes, Petri dishes, and flasks in your possession.

Sterilization of glassware. Dry heat is not as effective a germ destroyer as moist heat, but for certain purposes it is preferable. It is especially adapted for sterilization of empty. culture dishes, pipettes, and other glassware. Organic matter such as cotton must be carefully watched in sterilizing in dry heat, as it chars when heated above $150^{\circ} \mathrm{C}$. The slight browning of the cotton plugs in test tubes is an indication that the heating has been sufficient to destroy all bacteria present.

All material that can be subjected to dry heat is sterilized by heating to $150^{\circ} \mathrm{C}$. for one and one-half hours in a hot-air sterilizer. The latter consists of a double-walled box of sheet metal so provided with burners as to heat rapidly and uniformly. The glassware must be thoroughly dry before being placed in the sterilizer. In case the Petri dishes are placed in solid piles, it should be remembered that it requires a considerable period of time for the heat to penetrate into the 
inner dishes; hence heating the oven to $175^{\circ} \mathrm{C}$. and at once turning off the heat, as is sometimes recommended, is likely to result in incomplete sterilization. The sterilizer should not be opened until it has cooled to $60^{\circ} \mathrm{C}$. . If cold air strikes the hot glass, many dishes are likely to be broken.

After sterilization Petri dishes should not be opened until used. They should be handled as little as possible, as mold spores are likely to gain entrance; and in case they are to be

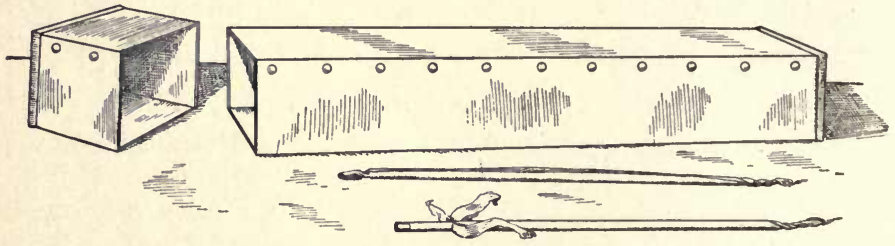

Fig. 2. Pipette Case and Pipettes

Case of sheet copper with slip cover; pipettes wrapped in tissue paper, on one of which the paper is torn to show the cotton plug in the mouth end

carried from the laboratory or are to be kept some time before using, they should be wrapped, before sterilizing, in pieces of newspaper.

The mouth end of the pipettes should be plugged with cotton, and the projecting fibers burned off so as not to interfere in using the pipette. The pipettes should be placed in the metal case, points down, the end of the case plugged with cotton, and the whole sterilized. In place of the metal pipette case the pipettes may be prepared for sterilization by wrapping each in tissue paper, the paper at the bottom being turned in and the mouth end twisted, so that the ends may be distinguished before removing the wrapper.

Exercise. Sterilize Petri dishes and pipettes.

Preparation of media. The media used in the bacteriological laboratory bears the same relation to bacteriology as does 
the standard solution to accurate work in chemistry. It is desirable that the media shall be uniform in composition and physical properties. This is, however, not wholly possible with the present methods. Much greater uniformity than usual might be attained, however, if standard ingredients and uniform methods were insisted on.

Ingredients of media. The following ingredients comprise those used in the preparation of the ordinary media:

Distilled water rather than tap water.

Infusion of meat, - Liebig's meat extract is often used as a substitute on account of its greater convenience and cheapness.

Peptone, - Witte's dry peptone prepared from meat.

Gelatin, - best French (gold label) brand. It is impossible to obtain gelatin that has a constant composition. It should be as free as possible from acids, and a 10 per cent solution, when prepared as directed, should not soften at $25^{\circ} \mathrm{C}$.

Agar, - commercial agar in threads of as high a grade as can be obtained. Agar is prepared from certain seaweeds of Ceylon and Japan, and is to be found on the market in the form of threads, square sticks, and as a powder.

Sugars, - saccharose, lactose, and anhydrous dextrose, used for the modification of standard media, should be as nearly as possible chemically pure. The two latter should be used in a powdered form.

Litmus, - the ordinary litmus cubes contain but a small amount of the pigment desired, azolitmin. It is preferable to purchase a purified form of litmus, which should be free from the reddish colorifics always found in the cubes, and which interfere with the sensitiveness of the indicator.

Reaction of media. The reaction of media is expressed with reference to the neutral point of phenolphthalein, and 
is stated in terms of the quantity of normal acid or alkali required to neutralize the medium. A medium having an acidity which would require the addition of $10 \mathrm{cc}$. of normal alkali to neutralize $1000 \mathrm{cc}$. is regarded as 1 per cent acid, and this condition is expressed as +1 per cent. If alkaline, so that $5 \mathrm{cc}$. of normal acid is required to render $1000 \mathrm{cc}$. neutral to phenolphthalein, the reaction is expressed as -0.5 per cent. In milk and also in the usual culture media the neutral point of phenolphthalein is not identical with that of litmus, due to the different behavior of these indicators with acid salts. The standard reaction of culture media for general use is +1 per cent.

Neutralization of media. The following reagents will be required: twentieth normal sodium hydrate $(\mathrm{N} / 20 \mathrm{NaOH})$, normal sodium hydrate $(\mathrm{N} / 1 \mathrm{NaOH})$, normal hydrochloric acid $(\mathrm{N} / 1 \mathrm{HCl})$, and an alcoholic solution of phenolphthalein ( 5 grams of the compound in 1000 cc. of 50 per cent alcohol) to be used as the indicator. The phenolphthalein should be dissolved in the alcohol and dilute $(\mathrm{N} / 20)$ sodium hydrate solution added until a very faint pink color is noted.

With a pipette place $5 \mathrm{cc}$. of the medium to be tested in a small flask or evaporating dish, add 45 cc. of distilled water, and boil one minute to drive off $\mathrm{CO}_{2}$, which affects the indicator. Add 5 drops of the indicator and titrate while hot with the $\mathrm{N} / 20 \mathrm{NaOH}$. A faint but distinct pink color marks the true end point. Each $0.1 \mathrm{cc}$. of $\mathrm{N} / 20 \mathrm{NaOH}$ used for $5 \mathrm{cc}$. of medium is equal to 0.1 per cent acid; thus a medium having an acidity of +1 per cent should require $1 \mathrm{cc}$. of $\mathrm{N} / 20 \mathrm{NaOH}$ for $5 \mathrm{cc}$. of medium. The final reaction should not differ from that desired by more than 0.2 per cent, i.e. the reaction should be between +0.8 and +1.2 per cent in case +1 per cent is desired. 
Filtering of media. Bouillon may be filtered through paper. The paper made by Schleicher \& Schüll, and known as No. $520 \mathrm{~N}$, is well adapted for the filtering of all kinds of media. The paper can be purchased in the form of folded filters.

Gelatin may be filtered by placing in a large funnel a small mass of copper turnings or clean excelsior ; over these a small piece of absorbent cotton ; then a layer of absorbent cotton large enough to reach to the top of the funnel. The layers of cotton should be thin so as not to retain much of the medium. Over the absorbent cotton place a piece of wet cheese cloth. Pour the medium into the funnel carefully. In case it has been correctly prepared, it will filter rapidly and be perfectly clear.

Agar may be filtered in the same manner as gelatin.

The media should be placed in test tubes, $7-8$ cc. in each tube. Extreme care should be taken to avoid wetting the upper part of the

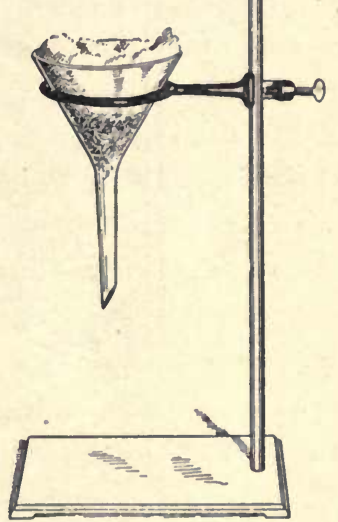

Fig. 3. Aprarates for Filtering Media

The absorbent cotton is supported by excelsior tube with the media, as this causes the cotton to adhere to the glass. A funnel arranged as illustrated in Fig. 4 is convenient; the glass tip should be slightly expanded at the lower end, and long enough so it can be held between the fingers and thus kept out of contact with the wall of the tube. The pinchcock should be closed rapidly so that no dripping will occur, and thus avoid soiling the mouth of the tube. The storage flasks should not be filled more than two thirds full; 


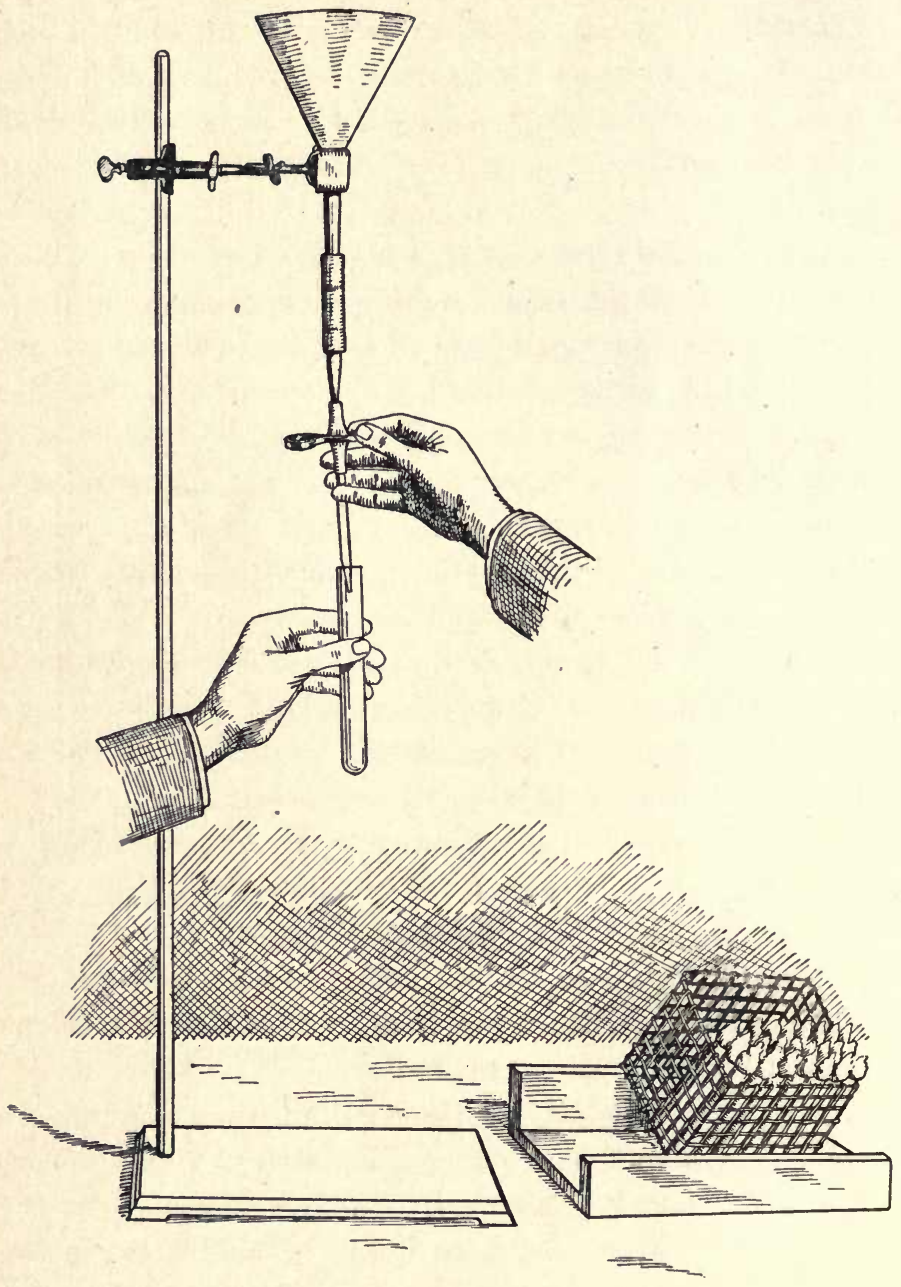

Fig. 4. Apparatus for tubing Menia

By holding the tip as shown, soiling the mouth of the tube with the medium is easily prevented 
if more nearly full, the cotton plug is likely to be wet by the medium during sterilization.

Plugging tubes. The test tubes filled with media are to be plugged with cotton at once. Ordinary roll cotton (not absorbent) is used for this purpose. A piece of cotton should be pulled from the roll, folded upon itself twice, the cotton compressed with the thumb and fingers and gradually forced into the tube. The plug should extend into the tube at least $1.5 \mathrm{~cm}$. and about the same distance above it, in order to protect the lip of the tube from dust and to enable one to remove the plug easily.

The tubes may also be plugged rapidly by pulling off a sufficient amount of cotton with a pair of dissecting forceps and thrusting it into the tube by means of the forceps.

Sterilization of media. Media in tubes or flasks is sterilized by an exposure to streaming steam at a temperature of $100^{\circ} \mathrm{C}$. from fifteen to sixty minutes, depending on the size of the containers, on each of three consecutive days, or by a single exposure to a considerably higher temperature, as in steam under pressure in a closed chamber (autoclave).

Various types of sterilizers are used. The Arnold is most convenient for ordinary laboratory purposes, as it heats rapidly and does not allow large quantities of steam to escape into the room. The media in tubes should be heated for twenty minutes on three consecutive days. With flasks the period of exposure should be extended in proportion to the size of the containers. The time should be computed from the moment when steam is freely generated in the sterilizer. During the interval between heatings, the media should be kept at room temperature, in order to allow the spores present to germinate. This system of sterilization is known as the intermittent or discontinuous method. 
In the autoclave a higher temperature is employed, and all resistant forms are destroyed in one heating. In order to be
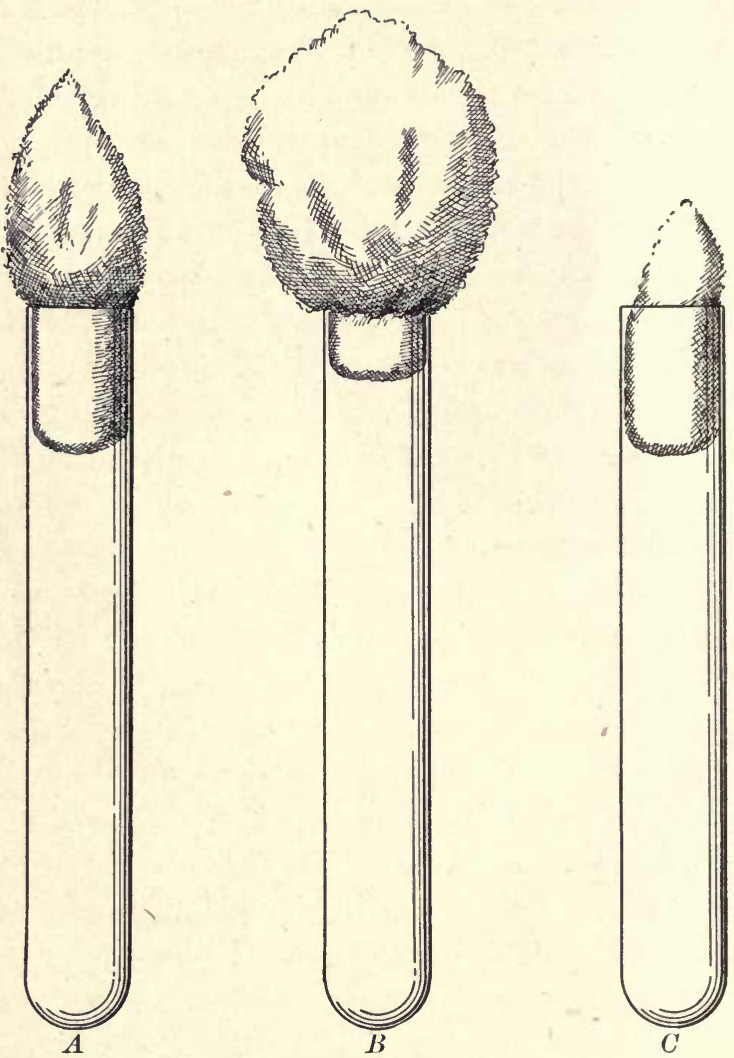

Fig. 5. Cotrox Plugs

$A$, a well-made plug; $B$, a plug that will easily become displaced; $C$, a plug that does not protect the mouth of the tube from dust

successful in the use of the autoclave certain points must be noted. Before using, care should be taken to observe whether there is a sufficient supply of water in the autoclave. After 
closing the apparatus the vent must be left open until the air is completely expelled by the generating steam, which should issue freely. In a mixture of air and steam the temperature does not correspond to the pressure noted. Five pounds of steam pressure produces a temperature of $109^{\circ} \mathrm{C}$. ; 10 pounds, $115^{\circ} \mathrm{C}$; 15 pounds, $121^{\circ} \mathrm{C}$. In all cases the apparatus must be allowed to cool off slowly and the vent opened very slightly; otherwise the pressure will decrease more rapidly than the temperature of the media. This will cause the media to boil so violently that the plugs may be forced from the containers and a portion of the media lost. This is especially true in the case of media in flasks. Media in tubes should be heated to $120^{\circ}$ C. (15 pounds pressure) for five minutes; in flasks the time must be extended.

Care of media. If possible the media should all be placed in tubes at the time it is prepared, and thus avoid the changes due to prolonged and repeated heating in the successive sterilizations necessary when the media is first sterilized in bulk and again when placed in tubes. After sterilization the media should be kept at room temperature for several days and examined from day to day. If any "specks" in 
gelatin or agar are detected, or the least cloudiness in bouillon appears, the medium is not sterile and must be immediately resterilized. As far as possible media that are not to be used at once should be stored in an ice box to prevent evaporation. In case of media that are to be held in storage for a considerable period, or incubated for some time at blood heat, the containers may be sealed by dipping the lower part of the cotton plugs in melted paraffin, or by placing a cap of tin foil over the cotton plug before sterilizing.

In each basket of culture medium should be placed a slip of paper stating the kind of medium, its reaction, the date of manufacture, and manner of sterilization. As far as convenient, culture media should be made in considerable quantities, so as to increase uniformity of conditions in culture work.

Preparation of broth. Nutrient broth is prepared as follows: Infuse 500 grams (18 ounces) of chopped lean beef in 1000 ce. of distilled water at refrigerator temperature for 24 hours. This should be done in a vessel of glass or enameled iron. Strain the infusion through cheese cloth or cotton flannel, pressing out the liquid until the meat appears dry. Place this infusion in a weighed media cooker, and add water to bring the weight of the infusion to 1000 grams. Add 1 per cent peptone (10 grams per liter) and warm gently, not above $50^{\circ} \mathrm{C}$. on the water bath, until the peptone is dissolved. Titrate two portions of $5 \mathrm{cc}$. each, and bring the reaction to +1 per cent by the addition of $\mathrm{N} / 1 \mathrm{NaOH}$. Titrate another portion of 5 cc. It will usually be found that the calculated amount of $\mathrm{N} / 1 \mathrm{NaOH}$ is not sufficient to bring the reaction to the desired point, due to the fact that the amount of $\mathrm{N} / 20 \mathrm{NaOH}$ necessary to neutralize 5 cc. of the medium diluted with $45 \mathrm{cc}$. of water does not exactly correspond with the amount of $\mathrm{N} / 1 \mathrm{NaOH}$ necessary to neutralize the 
undiluted medium. The reason for this is not known. Hence the titration must be made after the addition of the first quantity of $\mathrm{N} / 1 \mathrm{NaOH}$. Usually it will be found that a small quantity of the $\mathrm{N} / 1 \mathrm{NaOH}$ must be added.

After neutralizing, cook thirty minutes over boiling water; then boil for two minutes over the free flame, stirring constantly to prevent burning. Weigh and restore loss by evaporation, allow the medium to stand several minutes in order that the coagulated proteids may settle, filter through paper, titrate, and record final reaction. If the reaction varies more than 0.2 per cent either way from the normally accepted standard ( +1 per cent), it must be corrected.

The finished product should have a pale straw color, be perfectly clear, and on boiling a sample in a test tube should show no further precipitation of proteid matter. Place in flasks and tubes and sterilize in the autoclave.

\section{Summary :}

Infuse meat.

Weigh cooker and record weight.

Bring weight of infusion to 1000 grams.

Add 1 per cent peptone.

Warm to dissolve peptone, not heating above $50^{\circ} \mathrm{C}$.

Titrate and bring reaction to +1 per cent.

Cook thirty minutes over boiling water.

Boil two minutes over free flame, stirring constantly.

Restore weight.

Allow coagulum to settle.

Filter.

Test reaction and record.

Tube and sterilize.

Exercise. Each student will prepare 1 liter of bouillon. 
Preparation of gelatin. Prepare gelatin as follows: Infuse meat, strain, weigh cooker, and bring weight of infusion to 1000 grams, as in the preparation of bouillon. Add 1 per cent of peptone and 10 per cent of gelatin. Warm on water bath until peptone and gelatin are dissolved, not allowing the temperature to rise above $50^{\circ} \mathrm{C}$. Titrate and bring reaction to +1 per cent. Restore weight, taking into account the weight of the gelatin added. Cook thirty minutes over boiling water. Boil two minutes over free flame, stirring constantly.

Allow liquid to stand a few moments to settle. Filter through cotton (see p. 7). Test reaction, adjust if necessary. Record final reaction. Tube and sterilize. The filtering of gelatin is more difficult than in the case of broth. No trouble will be experienced if the filtering is not attempted until the coagulum is firm and solid and the liquid gelatin appears perfectly clear. If the proteids are not firmly coagulated, gelatin is very difficult to filter, as the fine sediment clogs the pores of the filter. If the cooking is continued until the albumen is completely coagulated and the funnel is arranged, as shown in Fig. 3, no trouble should be experienced.

Prolonged heating injures the solidifying properties of gelatin. If the sterilizing is done by the discontinuous method, the gelatin should be placed in cold water after each heating. If the autoclave is employed, the exposure should not exceed five minutes at $120^{\circ} \mathrm{C}$. (15 pounds pressure). After removal from the autoclave the gelatin should be cooled at once by placing in cold water. It is preferable to use but $7-10$ pounds pressure and continue the exposure for ten or twelve minutes.

Exercise. Each student will prepare 1 liter of gelatin. 
Preparation of agar. Infuse 500 grams of meat in $500 \mathrm{cc}$. of distilled water. Strain the infusion. Weigh the vessel, and bring weight of infusion to 500 grams. Add 10 grams of peptone. Warm over a water bath until peptone is dissolved. Heat 500 cc. of water in a weighed vessel until it is boiling rapidly. Add 15 grams of thread agar to the boiling water, continue the heating with constant stirring until the agar is completely dissolved, restore loss, and allow the whole to cool to $55^{\circ} \mathrm{C}$. The agar may also be dissolved by heating in the autoclave for ten or fifteen minutes at $120^{\circ} \mathrm{C}$. To the 500 grams of meat infusion, which should have a temperature of $45-50^{\circ} \mathrm{C}$., add the agar solution, keeping the temperature below $60^{\circ} \mathrm{C}$. Bring weight of mixture to 1000 grams. Titrate and bring reaction to +1 per cent. Heat thirty minutes over boiling water. Boil two minutes over a free flame, stirring constantly, as the agar is very likely to burn. Restore weight. Filter as in the case of gelatin. Test reaction, and adjust if necessary. Record final reaction. Tube and sterilize.

The filtering of agar is likely to be difficult unless the medium has been correctly prepared. The points to be noted are: (1) to obtain a perfect solution of the agar by prolonged boiling, or heating in the autoclave; (2) to produce a firm coagulation of the proteid matter by subsequent heating until the mass of the medium appears clear. The removal of the fine turbidity in agar can only be accomplished through its collection by the coagulating proteid, which is readily removed by filtration. If an attempt is made to filter before the medium is in proper condition, it will result in loss of time and material. If a heavy granular coagulum does not form upon prolonged heating, allow the medium to stand until the next day, break up the mass with a stirring rod, and melt over boiling water. This will usually result in a 
more complete flocculation of the proteid so as to permit ready filtration.

Agar is not injured by prolonged heating, as is gelatin. The finished medium should be clear when liquid, and slightly opaque when solid. It should be of a firm jelly-like consistency, not soft, mushy, or easily broken.

Exercise. Each student will prepare 1 liter of agar.

It frequently happens in the preparation of media that the material becomes seeded with resistant spore-bearing organisms. These do not come from the materials used in the media, but frequently from unclean utensils. All utensils — media cookers, funnels, pipettes, etc. — should therefore be washed with hot water and drained as soon as possible, in order to free them from the adhering media. If allowed to stand, germ growth takes place in this material, and the utensils become so abundantly seeded as to make sterilization of the media prepared in such vessels difficult or impossible.

Meat infusion should be used whenever possible for media, but beef extract can be substituted, 3 grams of Liebig's Beef Extract being used for each liter of medium. Egg albumen must then be used as a clearing agent, to remove the finely divided material. The white of one egg for each liter of medium should be added to the distilled water before adding the other ingredients. The albumen should be well beaten and thoroughly mixed with the water. Then proceed as when meat infusion is used.

Preparation of milk. The milk to be used as a culture medium should be as fresh as possible. It should not have an acidity above 0.18 per cent calculated as lactic acid. The normal acidity of perfectly fresh milk ranges from 0.12 to 
0.18 per cent of lactic acid, equaling +1.3 to +1.6 per cent on the arbitrary scale ordinarily employed for media.

On heating, the acidity is reduced by the precipitation of the calcium salts and the expulsion of the $\mathrm{CO}_{2}$, so that a milk having an acidity of 0.18 per cent calculated as lactic acid will show not more than +1.2 per cent when tested after sterilization by the usual method. It is possible to neutralize milk showing a high degree of acidity, but it is not advisable, since within certain limits the acidity is a measure of purity, and even under the best of conditions milk is very difficult to sterilize. The whole milk may be allowed to cream in a refrigerator by gravity, and the skim milk siphoned off, or, preferably, the milk may be skimmed in a centrifugal machine or cream separator.

Milk is used either as plain milk or in the form of litmus milk prepared by the addition of a litmus solution. The litmus solution should be added to the milk before tubing, and in sufficient amount to give it a decidedly blue color. Milk is preferably sterilized by the discontinuous method. Litmus is easily reduced at $100^{\circ} \mathrm{C}$. by the action of the milk sugar. Thus when litmus milk is removed from the sterilizer, it may appear almost colorless. The color soon reappears on exposure to the air, due to the oxidation of the reduced indicator. The sterility of the milk must be tested by allowing it to stand a week, if possible, before using;

Preparation of sugar media. Gelatin, agar, and bouillon are often amended by the addition of sugars (saccharose, dextrose, and lactose). Sugar media should be sterilized by the discontinuous method, and the reaction should be neutral to phenolphthalein. Saccharose is, however, the only one of the three sugars easily affected, being changed in part (inverted) to dextrose and levulose when heated in acid solutions. Lactose 
is not so easily decomposed, and media containing it may be sterilized in the autoclave even when acid. When it is desirable to test the properties of an organism with reference to its conduct towards certain sugars, care should be taken to have no other sugar present in the medium than the one added, and to have this present in an unchanged form.

Media prepared from meat infusion will contain some muscle sugar (inosite). This may be removed from the infusion by seeding the same with an organism able to ferment this sugar (B. coli communis), incubating from sixteen to twenty hours at $37^{\circ} \mathrm{C}$. and then proceeding as usual in the preparation of the medium.

Preparation of litmus solution. The litmus ordinarily purchased varies so widely in its content of the pigment desired, azolitmin, that it is impossible to give definite directions for its preparation. Ten grams of Kahlbaum's or Schuchardt's litmus powder are extracted with 500 cc. of water by placing over night in a warm place; then filter and tube. Sterilize by the discontinuous method.

Preparation of water blanks. For dilution purposes in quantitative work sterile water must be employed. It is customary to use the so-called physiological salt solution, prepared by the addition of 0.6 per cent of common dairy salt to distilled water, in place of the distilled water which may exert an injurious effect on the bacteria by reason of its osmotic properties.

Various modifications of the above media are used in bacteriological work, as well as many other types of media, such as potato, blood serum, egg, etc. These, however, are not usually employed in ordinary dairy work, and for their preparation and use the student is referred to books on general and medical bacteriology. 
Quantitative analysis of milk. The number of bacteria present in any material is determined by making "plate cultures" from it. A definite quantity is placed in a Petri dish, and a tube of one of the liquefiable solid media is poured into the Petri dish and intimately mixed with the milk. The liquid medium spreads in a thin layer over the bottom of the plate, which is placed on a level surface to cool, thus allowing the medium to solidify. The bacterial cells distributed in the liquid medium are held in place when the mediuın solidifies. The resulting growth thus appears as distinct spots, called "colonies," each of which is the progeny of a single type of organism, although not necessarily of a single cell. The counting of these colonies after a sufficient time has elapsed for their growth will give the approximate number of living bacterial cells present in the original substance at the time the plate cultures were made. This culture method, originally devised by Koch, has been of greatest value in placing bacteriological study on a strictly scientific foundation.

It is impossible by any method yet devised to determine the absolute number of bacteria present in any substance, which, like milk, contains under natural conditions a variety of forms differing in requirements for their growth. The colonies that appear on the plate cultures represent the organisms that, under the given conditions of food, temperature, and air supply, find favorable conditions for development. If another food substance is used, if the reaction of the medium is varied widely, or if the temperature is changed, a different class of bacteria will be found on the plate cultures. Hence the plate method of determining the number of bacteria in milk or any substance is not an absolute one, and one which gives comparable results only under constant conditions. 
Each colony represents a living bacterial cell in the substance plated, only when the cells are uniformly distributed throughout the liquid and no clumps of cells persist. This condition is impossible to attain in viscous liquids and in those in which growth takes place; hence, on culture plates, many colonies represent the progeny of a clump of cells, numbering many score or more, instead of being the result of the growth of a single cell. The results obtained from the examination of any sample will thus vary with the care used in obtaining an even distribution of the bacteria. If a sample of milk is allowed to stand for twenty-four hours and plate cultures prepared before it has been shaken, and another set after the milk has been shaken for several minutes, the results will vary widely, due to the more or less complete rupture of the cell clumps of the bacteria.

If the colonies on the plates are very numerous, they will consist mainly of those kinds that, under a given environment, find most favorable conditions for growth. Those finding less favorable conditions are inhibited. If lactose-agar plates are seeded with a sample of sour milk and kept at $25^{\circ}-30^{\circ} \mathrm{C}$, the ordinary sour milk or lactic-acid bacteria will grow very rapidly, and the putrefactive bacteria will not develop; but if the cultures are made with gelatin containing no milk sugar, the sour milk bacteria will develop very sparsely, or not at all, and only the other typess will be found on the plates.

If the colonies are less numerous, so that each is not within the zone of influence of other developing colonies, this inhibition will not take place. Generally such inhibition or "antagonism" is caused by the effect of the soluble byproducts of one form diffusing throughout the medium.

For ideal results each colony should develop as though it were the only colony on the plate. This condition is of course 
difficult to obtain. The extent of the zone of influence of a colony depends upon the organism and the medium. Inhibition by overcrowding is negligible when the number of colonies ranges from 100 to 200 on a $90-\mathrm{mm}$. plate.

If larger numbers are present, this inhibitory action becomes pronounced and a reduction in total content is to be observed. This error, as well as tedious enumeration of very small colonies, makes it preferable to have properly prepared plate cultures. In plates prepared from pure cultures of bacteria, i.e. organisms all of one kind, this inhibiting tendency is much less important.

Dilution. The number of bacteria present in any given material, as, for instance, milk, is of course unknown. Hence, in preparing plate cultures, in order to have the proper number of colonies it is necessary to vary considerably the quantities of milk used in the plates. The milk should be diluted with sterile water or salt solution (water blanks). The extent to which the dilution must be carried cannot be determined with exactness, but with experience the analyst can judge from the age of the milk, the conditions under which it was produced, the temperature at which it has been kept, and, by determination of the acidity, the dilutions which are most likely to give satisfactory results.

In the case of perfectly fresh milk, plates should be made with $1 / 10,1 / 100$, and $1 / 1000 \mathrm{cc}$; ordinary market milk $1 / 100,1 / 1000,1 / 10,000 \mathrm{cc}$; milk having an acidity over 0.2 per cent, $1 / 1000,1 / 10,000$, and $1 / 100,000$ cc. In the case of sour milk and cream it will be necessary to use still greater dilutions.

The various dilutions are obtained by the use of 9-cc. and 99-cc. water blanks in test tubes and 150-cc. Erlenmeyer flasks. To obtain $0.1 \mathrm{cc}$. of milk, $1 \mathrm{cc}$. is placed in a 9-cc. 
water blank by means of a sterile 1-cc. pipette, the liquid well mixed, and 1 cc. of the mixture taken. To obtain $1 / 100 \mathrm{cc}$. of milk, $1 \mathrm{cc}$. is placed in $99 \mathrm{cc}$. of water and $1 \mathrm{cc}$. taken; $1 / 1000$ cc. of milk is obtained by carrying $1 / 100$ cc. to a 9 -cc. water blank and using $1 \mathrm{cc}$. of the mixture. Still higher dilutions are obtained in a similar manner. In each plate is placed the same amount of the mixture of milk and water, $-1 \mathrm{cc}$; thus the extent to which the medium is diluted is constant. A clean pipette must be employed for each dilution.

Media. A variety of media are used for the quantitative analysis of milk. Each has certain advantages and disadvantages. Gelatin melts at temperatures above $25^{\circ} \mathrm{C}$., and is liquefied by certain kinds of bacteria. If these forms are numerous, the entire mass of gelatin will be rapidly liquefied and no definite results obtained. Agar melts at $96^{\circ}-$ $99^{\circ} \mathrm{C}$. and does not solidify again until it has cooled to $39^{\circ}-40^{\circ} \mathrm{C}$. It is not liquefied by any of the ordinary bacteria. Gelatin can therefore be used only at room temperature, while agar cultures may be incubated at blood heat. Lactose agar is preferable for quantitative analysis of milk.

Cooling. After the liquid medium has been poured into the plates they must be tilted from side to side in order to distribute the medium evenly over the entire inner floor of the dish. This must be done before the medium begins to solidify, so that the appearance of the plate shall not be marred by a rough surface. This is easily accomplished with gelatin, as it cools slowly. With agar the medium must be allowed to cool to $43^{\circ}-45^{\circ} \mathrm{C}$. before it is inoculated, and since it solidifies at $39^{\circ}-40^{\circ} \mathrm{C}$., it requires more careful manipulation to properly prepare cultures with this medium. More uniform distribution can be had with agar if before adding the medium the plates are slightly warmed by holding them 
in the hand and passing them quickly through the flame of a Bunsen burner. After the culture medium is distributed the plates should be placed on a perfectly level surface to solidify, in order that the culture medium may be uniform in thickness and the colonies evenly distributed.

Incubation. Nearly all forms of bacteria found in milk grow at room temperature, about $20^{\circ} \mathrm{C}$, although higher temperatures hasten the growth of many kinds.

Two temperatures are ordinarily used in bacteriological laboratories, $-20^{\circ} \mathrm{C}$. (room temperature) and $37.5^{\circ} \mathrm{C}$. (blood heat). In order to maintain a constant temperature an apparatus known as a thermostat or incubator is used. A temperature of $37.5^{\circ} \mathrm{C}$. is maintained by a gas flame, the gas supply being regulated by a thermoregulator. A constant temperature of $20^{\circ} \mathrm{C}$. is difficult to maintain, since under summer conditions room temperature often exceeds this limit. If an abundant supply of cold water is available, the thermostat may be kept cool, i.e. at a temperature below $20^{\circ} \mathrm{C}$., and then heated with a small, regulated gas flame.

Gelatin cultures must always be incubated at temperatures below $25^{\circ} \mathrm{C}$. Agar cultures may be kept at any desired temperature up to the maximum for bacterial growth, which for most species does not exceed $40^{\circ}-45^{\circ} \mathrm{C}$.

The proper time of incubation, i.e. the period between the preparation and the counting of the plates, will depend upon the temperature. If $37.5^{\circ} \mathrm{C}$. is used, the number of colonies will not materially increase after forty-eight hours incubation. The individual colonies will, however, increase in size and make the counting easier. This is especially true if the plates are incubated for another forty-eight hours after they are removed from the $37^{\circ} \mathrm{C}$. incubator. Plates kept at $20^{\circ} \mathrm{C}$. should not be counted before three days, and the maximum 
number of colonies will not appear in less than six or eight days. It is, however, often advisable to secure results before the expiration of such a long period of incubation, and for comparative purposes a shorter time than the maximum here given is frequently used as a basis for official counts.

Agar on solidifying expresses a slight amount of water. This often collects on the surface of the medium in a thin film, allowing the formation of a spreading surface growth. Frequently a thin, nearly transparent growth may spread between the under surface of the culture medium and the glass. If freshly prepared plates are placed immediately in a $37^{\circ} \mathrm{C}$. incubator, evaporation from the culture medium occurs, which permits of condensation on the cover of the dish, and the moisture sometimes collects to such an extent that droplets of water fall on to the medium, causing the formation of a spreading growth and seriously interfering with subsequent counting. To obviate this, the plates may be inverted in the $37^{\circ} \mathrm{C}$. incubator. Covers made of porous earthenware are also useful in preventing this trouble.

Counting. To enumerate the developing colonies, the cover is removed from the culture dish and the plate placed on a black surface, which is divided into appropriate divisions, in order to facilitate counting (Frost's modification of the Jeffer's plate counter is most satisfactory). The counting should be done by the aid of a hand lens, magnifying $5-8$ diameters. When possible, the entire plate should be counted. Where the colonies are too numerous, an estimate may be made by counting a representative portion of the plate, and from the data obtained calculating the total number of colonies on the plate. This process is, however, often unsatisfactory, especially if the colonies are not uniformly distributed. If the plate was not allowed to solidify in a level position, 
the colonies will be abundant on one side and sparse or absent on the other, making it difficult to secure a correct approximation. In case the count to be made is not the final one, or if the culture is to be used for other purposes, the cover of the dish must not be removed during the counting process.

A number of plate cultures must be made from each sample of milk. Not all of these are to be counted, but only those having the most desirable number of colonies. If plates have been made with $1 / 100,1 / 1000$, and $1 / 10,000$ cc. of milk, the colonies on the first plate of the series may be so numerous as to render counting difficult or impossible. The next plate, containing only $1 / 10$ as much, may have several hundred, while the final one of the series, made with $1 / 10,000$ cc., should have only a few score of colonies. To give reliable and consistent results plate cultures should show between forty and two hundred colonies. When the number of colonies is much in excess of two hundred, inhibition of one form by another takes place, and increases with an increasing number of colonies. The maximum number of colonies that will develop on a plate ranges from ten to fifteen thousand. If the material used in preparing the plate culture contained a greater number of organisms, the results will not be accurate. If the colonies are few in number on the plate, it is difficult to obtain concordant results. For these reasons plates which do not approximate the number of colonies considered as desirable should be discarded. The average of all plates counted should be taken and the results expressed as the number of bacteria per cubic centimeter in the milk examined.

Expression of results. Since it is impossible to determine with absolute accuracy the number of bacteria present in any 
substance, fictitious accuracy should be avoided in the expression of the results. In reporting the results of the examination of ordinary market milks, the Committee on Standard Methods of Milk Analysis of the American Public Health Association has made the following recommendation:

From 50,000 to 500,000 bacteria per cubic centimeter report results to nearest 50,000 .

From 500,000 to $1,000,000$, to nearest 100,000 .

From $1,000,000$ to $2,000,000$, to nearest 200,000 .

From 2,000,000 to 5,000,000, to nearest 500,000.

Above 5,000,000, to nearest 1,000,000.

The error with present methods, even with very careful work; amounts to $10-15$ per cent, so it is evident that the above scheme is sufficiently accurate.

In reporting the results of very clean milk (certified and sanitary milk) the exact figures should be given, neglecting the first significant figure, as 510 bacteria per cubic centimeter instead of 514 .

Recapitulation of preparation of plate cultures. The procedure for the examination of a sample of milk from which plates are to be made containing $1 / 100,1 / 1000$, and $1 / 10,000$ cc. of milk is as follows. Shake sample twenty-five times.

Mark two 99-cc. water blanks 1 and 3 respectively, and mark one 9-cc. water blank 2.

With a sterile pipette transfer to blank No. 1,1 cc. of milk, and return pipette to milk. Replace the cotton plug in the water flask and shake well. No harm will be done if the cotton becomes wet.

With another sterile pipette, transfer 1 cc. from flask No. 1 to No. 2, and also 1 cc. from No. 1 to No. 3. Return pipette to No. 1. The cotton plug need not be replaced. Mix Nos. 2 and 3 , and in each place a sterile pipette. 
Label six sterile culture dishes with the laboratory number of the sample of milk, date, and quantity of milk to be placed in each. Transfer to each of two Petri dishes $1 \mathrm{cc}$. of the first dilution ( $1 / 100 \mathrm{cc}$. of the original milk), to two more dishes $1 \mathrm{cc}$. of the second dilution ( $1 / 1000 \mathrm{cc}$.), and to the two remaining dishes $1 \mathrm{cc}$. of the third dilution $(1 / 10,000 \mathrm{cc}$.).

In placing the diluted milk in the dish lift the cover at one side, place the tip of the pipette on the bottom of the dish and allow the desired quantity to run out.

Do not remove the cover completely.

Do not allow the pipette to touch the desk or any other unsterile object.

In a metal cup partly filled with water place seven tubes of lactose agar. Heat over a Bunsen burner until the agar is completely melted.

Place a thermometer in the cup, cool to $45^{\circ} \mathrm{C}$. by adding cold water, and allow the tubes to stand five minutes at this temperature.

Remove a tube, wipe off the water, remove the cotton plug. Flame the open end of the tube in a Bunsen flame.

Lift one side of the cover of the culture dish, pour in the culture medium, replace the cover, mix the medium with the diluted milk by tipping the Petri dish from side to side, and place the dish on a level surface.

Incubate at $37^{\circ} \mathrm{C}$. for forty-eight hours. ,

Count cultures and record results.

Results obtained from duplicate plates should check within 15 per cent.

Pour the seventh tube of melted agar into an empty, sterile culture dish as a check. Incubate. If due care has been exercised and medium and glassware are sterile, no colonies should develop in this culture. 
Exercise. Each student will make a quantitative examination of three samples of milk: (1) fresh milk; (2) ordinary market milk; (3) milk having a high acidity (0.3-0.4 per cent).

The dilutions to be used in the examination of the different milks should be indicated by the instructor.

Arrangement of data. The complete data should be arranged in tabular form as far as possible, so as to facilitate the examination of the results. The following may serve as an example of arrangement:

\begin{tabular}{|c|c|c|c|c|c|}
\hline $\begin{array}{l}\text { LABORATORY } \\
\text { NUMBER OF } \\
\text { SAMPLE }\end{array}$ & $\begin{array}{l}\text { AMOUNT OF } \\
\text { MILK }\end{array}$ & $\begin{array}{c}\text { COLONiES } \\
\text { AT } 48 \\
\text { Hours }\end{array}$ & $\begin{array}{c}\text { Colonies } \\
\text { AT } 96 \\
\text { Hours }\end{array}$ & $\begin{array}{c}\text { Number } \\
\text { Per CUBic } \\
\text { CENTIMETER }\end{array}$ & $\begin{array}{l}\text { NuM- } \\
\text { BER OF } \\
\text { KINDS }\end{array}$ \\
\hline I & $\begin{array}{l}1 / 100 \text { cс. } \\
1 / 1000 \text { cc. } \\
1 / 10,000 \text { сc. } \\
1 / 100 \text { cc. } \\
1 / 1000 \text { cc. } \\
1 / 10,000 \text { cc. }\end{array}$ & $\begin{array}{l}\text { Not counted } \\
\text { Not counted } \\
200 \\
\text { Not counted } \\
110 \\
14\end{array}$ & $\begin{array}{c}208 \\
121 \\
15\end{array}$ & $\begin{array}{c}2,000,000 \\
110,000 \\
140,000\end{array}$ & 4 \\
\hline
\end{tabular}

Method for comparative results. When it is desirable to determine merely the relative number of bacteria in a number of samples, as, for example, in sweet and ripened cream, a different method may be used, which is sufficiently accurate for the purpose.

A standard-sized loop is made of No. 27 platinum wire by making the loop around an eight-penny common nail $\left(10 \frac{1}{4}\right.$ wire gauge). The loop should be made as illustrated (Fig. 7). If it is always used in the same way, i.e. immersed to the same depth in the liquid, removed quickly, and the lumen of the loop kept parallel to the surface of the liquid, a double convex drop of liquid will be removed, which will contain an approximately uniform quantity of milk ( 0.008 to 0.012 grams). High dilutions can thus be obtained by the use of small water blanks ( 5 cc.), and the use of many pipettes avoided. 
Qualitative analysis of milk. It is frequently desirable to determine the number of different kinds' of bacteria in a sample of milk, as, for instance, acid-producing organisms, organisms liquefying or not liquefying the gelatin. For these purposes media must be used which will permit of the development of the desired species.

In order to determine the number of acid-producing and liquefying organisms, litmus lactose gelatin may be used, proceeding exactly as in the case of the quantitative examination of milk, except that to each tube of lactose gelatin, after melting, is added with a sterile pipette sufficient sterile litmus solution to give the medium a decidedly blue color. The acidproducing bacteria are easily differentiated on this medium by their red color unless the plates are so thickly seeded with acid colonies that the entire mass of the medium is changed. For qualitative analysis thickly seeded plates are of little value.

This medium also divides the bacteria which develop into two classes, namely: (1) liquefiers, - those which develop ferments capable of permanently rendering the gelatin medium liquid; (2) those unable to produce this change. The liquefiers are further differentiated into those which perform this change very rapidly and those which produce a restricted and often deepened pit of liquefied material closely surrounding the colony.

The differentiation of the bacteria can be carried further by a study of individual colonies. These vary in size and appearance with different organisms, and the differences are more or less constant. Especial use is made of these colony characteristics in the detailed study and identification of the various kinds of organisms.

Exercise. Prepare litmus-lactose-gelatin plates from the samples of milk furnished. Incubate at $20^{\circ} \mathrm{C}$. Count the number of acid 
colonies, the number of liquefying and nonliquefying colonies on the gelatin plates; and express the results as the number of each contained in a cubic centimeter of the milk.

Isolation of pure cultures. A pure culture is one which contains but a single kind of organism. Under natural conditions the bacterial flora of any substance is made up of a number of kinds. If it is desired to study the different kinds in detail, a separation must be made and each obtained in "pure culture." Each colony on the culture plate represents the progeny of a single cell or of a number of adherent cells of one kind; hence the cultures obtained from a single colony are termed "pure cultures." If from the isolated colony further cultures are made in proper media, the morphological and physiological study of the organism can be carried out.

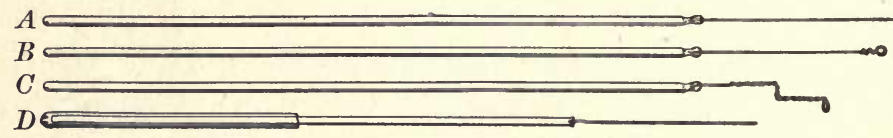

Fig. 7. Platinum Needles

$A$, a straight needle; $B$, an ordinary loop; $C$, a standard loop (the bend indicates the depth to which the loop is dipped in the liquid each time so as to remove equal amounts); $D$, Ravenel's needle

Platinum needles. The bacteria are transferred from one culture vessel to another, or are handled by means of platinum needles. These are made of No. 25 or 27 gauge platinum wire for ordinary work. For special purposes lighter or heavier wire is used. A piece $50 \mathrm{~mm}$. long is fused into a glass rod or tube about $180 \mathrm{~mm}$. long and $2.0-2.5 \mathrm{~mm}$. diameter. The danger of the glass cracking at the point of insertion of the wire when the needle is heated can be lessened by fusing on the end of the ordinary glass rod a bead of readily fusible glass, into which the wire is inserted. Jena glass, No. 397, III, is excellent for this purpose. 
All trouble can be avoided by the use of the needle handle devised by Raverfel. An aluminum rod of $2.5-3 \mathrm{~mm}$. diameter is procured and cut into pieces $150-200 \mathrm{~mm}$. long. In the end of each rod a hole is drilled with a small drill. The platinum wire is inserted into the hole and the end of the rod hammered lightly so as to close the hole about the wire. The aluminum rod may be inserted in a piece of glass tubing, so that it can be more easily handled.

Each student should have two inoculating needles, (1) one in which the wire is straight and is used for puncture inoculations, (2) and another in which a loop is formed at the free end, which is used in making transfers from liquid cultures. The loop should be made around an eight-penny common nail which has a gauge of $10 \frac{1}{4}$. The needle must always be heated to red heat for its entire length, and the lower part of the glass rod be passed through the flame several times in order to sterilize the needle before use. The wire should be allowed to cool for a few seconds before it is used. After using, it must be again heated to red heat to sterilize it before being laid down. The habit of heating immediately before and after using must be acquired, so as to become practically an involuntary act. If this habit is acquired, much subsequent trouble will be avoided. The lower part of the glass should not be soiled with media. A "loopful" means all the fluid the loop can hold. This should be a biconvex mass, not merely a film covering the lumen of the loop.

Exercise. Each student will prepare a needle and a standard loop.

Plate cultures. In testing the purity of a culture or in the. isolation of the forms present in a mixture of bacteria plate cultures must be prepared. This is done, in case a culture is to be tested for its purity, by melting three agar or gelatin 
tubes, cooling to $42^{\circ}-45^{\circ} \mathrm{C}$, and marking them 1,2 , and 3 . With the sterile needle transfer a small amount of the growth to be tested to the first tube of melted medium, replace the plug, sterilize the needle, and mix the material by rotating the tube between the hands. From this tube transfer three loopfuls to the second tube, mix, and again carry three loopfuls to the third tube. Pour each tube into a sterile Petri dish, spread, and allow the medium to solidify. Incubate. On one of the three dishes will usually be found such a number of colonies that they will be able to attain the maximum size and development. When preparing plates the probable number of living organisms present must be considered and the amount of original material taken, and the number of loopfuls transferred from one tube to another should be taken accordingly. Three plates should always be made. An effort to economize in material by making one or two plates is very apt to end in failure. Sometimes one tube of medium may be saved by using a water blank for the first transfer, as this culture is generally so abundantly seeded that it is worthless for study.

Study of plate cultures. A macroscopical and microscopical examination of the colonies appearing on the plates should be made, as the general appearance is more or less constant and characteristic for each kind of bacteria. The microscopical examination should be made by inverting the Petri dish on the stage of the microscope and studying the colonies with a low power ( $16 \mathrm{~mm}$. objective). The following points should be noted in the examination: surface colonies, i.e. colonies which are wholly or partially on the surface of the medium: form of colony; size of colony; surface elevation; topography of surface; microscopic internal structure of colony ; microscopic structure of edge of colony; color determined both by 
transmitted and reflected light. Specific or technical terms are used to describe these characters. These the student should learn at an early date, so as to acquire habits of precision in the matter of species description. In the examination of deep colonies, i.e. imbedded in the medium, note the form, size, microscopic structure, consistency, color, change in surrounding medium. Deep colonies as a rule are far less characteristic than the superficial ones.

Test-tube cultures. The pure cultures are maintained and are studied in "tube cultures." The transfers are made by means of the platinum needles from the colony on the plate culture to tubes containing various kinds of media. A large part of the detailed study of an organism consists in noting the character of the growth and the changes produced in various media in the absence of all other forms, i.e. in pure culture, by the organism in question.

Fishing. In the preparation of test-tube cultures from the plate cultures care should be taken in the selection of the colony from which the tube culture is to be inoculated. The plate should be examined macroscopically and a ring drawn with a colored wax pencil about each colony to be used. The colony and its immediate neighborhood should then be examined under the low power of the microscope, in order to determine whether there are any near-by microscopic colonies. Mark the colonies which show a clear zone of several millimeters in width about them. Sterilize the platinum needle, remove the cover of the Petri dish, and touch the colony with the end of the needle. With the infected needle inoculate the test-tube culture; mere contact of the inoculating needle with the surface of the colony is amply sufficient to obtain cells enough to seed the new culture. It is not necessary to transfer a visible amount of 
growth. From the tube culture after the incubation inoculations can be made into various media.

Liquid cultures. These are made in nutrient broth, milk, etc.

Stab cultures are made in solid gelatin and agar by infecting the end of the needle with a bit of the growth from the colony or previous culture, and thrusting the needle for

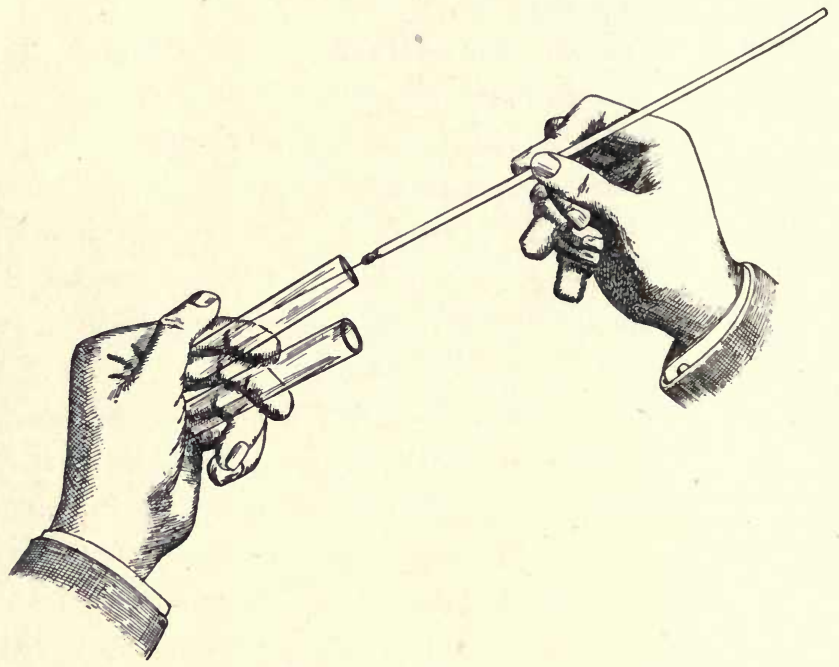

Fig. 8. Inoculating Tube Culteres

The cotton plugs should be held in such a manner that the lower part will not come in contact with the hand, and so they can be easily replaced

nearly its entire length into the medium and withdrawing. The gelatin should not crack on insertion of the needle; if it does so, a fresh tube must be melted in warm water and resolidified in cold water. The cracking is due to the drying out of the upper layers of the gelatin in the tube.

The culture of the organism must be kept pure. During the various manipulations the cultures are constantly exposed 
to contamination from the air and from the surrounding objects. All operations must be carried out in such a way as to permit no contamination to take place. In inoculating a culture tube the cotton plug must be removed and held in such a way that the portion which is inserted into the tube shall not come in contact with any object. The tube or tubes should be held as illustrated in Fig. 8. If any cotton adheres to the mouth of the tube, it should be removed by passing the tube through the flame of a Bunsen burner. The tubes should be kept open no longer than necessary, and the needle sterilized just before using.

Streak cultures. Agar may be allowed to solidify with the tubes in an inclined position, thus giving a large sloping surface that is inoculated by drawing over it in a single stroke the infected needle or loop. Care should be used not to cut or break the surface of the medium. Potato, blood serum, and egg are used in the same manner. Gelatin can be used in this way for organisms that are unable to liquefy it.

Study of test-tube cultures. The following points should be noted in the tube cultures:

Nutrient broth: conditions of fluid, - whether clear or turbid; character of turbidity; amount of sediment; surface pellicle; odor.

Gelatin stab cultures : character of growth along line of inoculation; surface growth; extent of liquefaction; shape of liquefied area.

Agar stab cultures : character of growth along line of inoculation; surface growth.

Streak cultures: form; size; surface elevation; topography of surface; color ; consistency; odor; luster; change in medium.

For list and definition of the terms employed in the description of the cultural characters of an organism, see Frost's 
Laboratory Bacteriology, and Chester's Determinative Bacteriology, and also Appendix B.

Exercise. Each student will prepare from the mixed culture furnished him a set of plates, using agar as the culture medium, and will isolate the different organisms in pure culture and make a detailed cultural study of the same. From the different types of colonies on the agar plates inoculate agar slopes. Incubate these from twentyfour to forty-eight hours, select two of each type for further study, making agar-stab, gelatin-stab, and nutrient-broth cultures from each; also prepare gelatin plates. 


\section{CHAPTER II}

\section{MICROSCOPICAL TECHNIQUE}

The student of bacteriology should be familiar with the use of the compound microscope. If experience with this instrument has been gained in other fields of biology, the peculiar methods of bacteriology are easily acquired. If, however, the student has had no previous training, he should familiarize himself with the construction and purposes of the different parts of the microscope by a perusal of the booklets issued by various optical companies on the use and care of the microscope, and by a study of the first three chapters in Winslow's Elements of Applied Microscopy.

The microscope and its accessories. The microscope for bacteriological purposes should be provided with both a coarse and a fine adjustment, with three objectives, $-16 \mathrm{~mm}$. $\left(\frac{2}{3}\right.$ inch $)$, $4 \mathrm{~mm}$. ( $\frac{1}{6} \mathrm{inch}$ ), and $2 \mathrm{~mm}$. ( $\left.\frac{1}{12} \mathrm{inch}\right)$, - equivalent focus, two oculars, $-25 \mathrm{~mm}$. (1 inch) and $50 \mathrm{~mm}$. (2 inch), - a triple nose piece, so that the objectives can be easily changed, and an Abbe substage condenser, with iris diaphragms both above and below the lens of the condenser. The Abbe condenser and the $\frac{1}{12}$-inch oil-immersion lens are indispensable to the ordinary equipment of a compound microscope for bacteriological work.

In the examination of bacteria high powers of magnification must be used (500-800 diameters). In order to secure these magnifications very small lenses must be employed, thus restricting the amount of light passing through them. The modifications of the ordinary compound microscope, to 


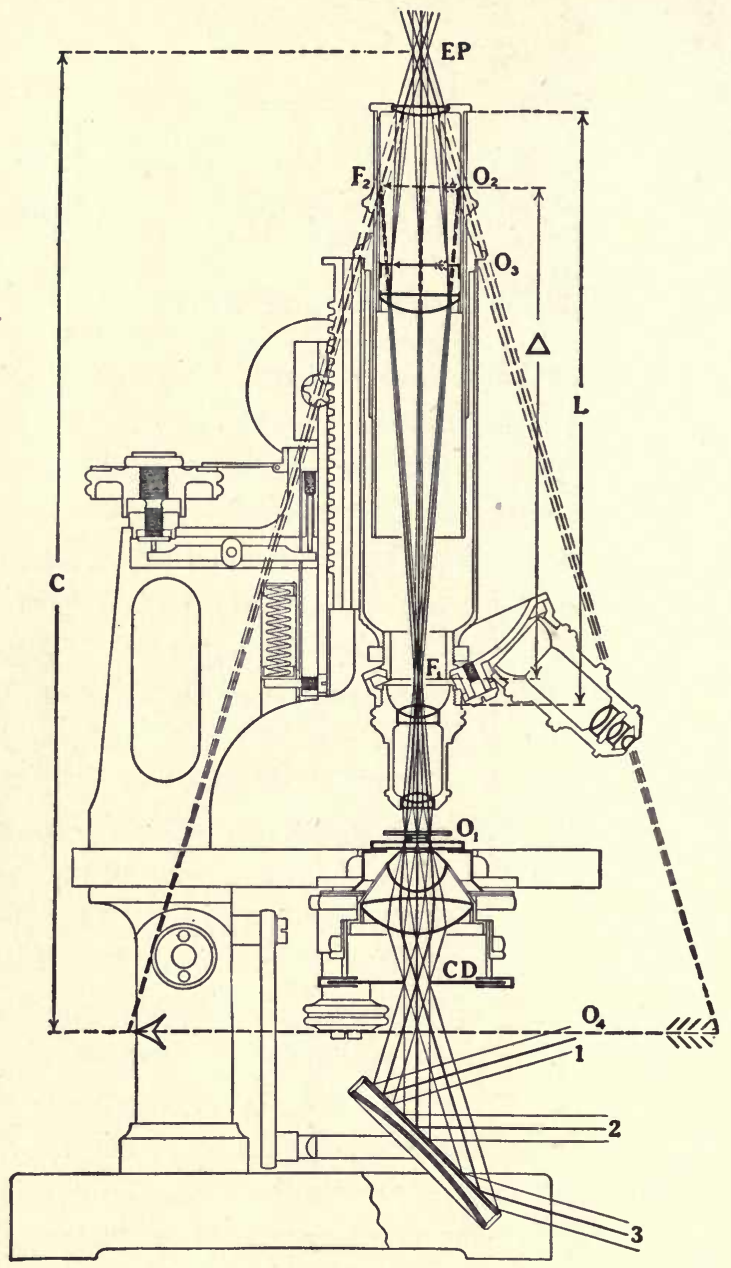

Fig. 9. Section of a Microscope

$\mathrm{O}_{1}$, object; $\mathrm{O}_{2}$, real image in $\mathrm{F}_{2}$, transposed by the collective lens, to $\mathrm{O}_{3}$, real image in eyepiece diaphragm; $O_{4}$, virtual image formed at the projection distance $C, 250 \mathrm{~mm}$. from $E P$, eye point; $C D$, condenser diaphragm; $L$, mechanical tube length $(160 \mathrm{~mm}.) ; 1,2,3$, three pencils of parallel light coming from different points of a distant illuminant, for instance, a white cloud, which illuminate three different points of the object. (After Bausch \& Lomb Optical Co.) 
adapt it to bacteriological work, are for the purpose of concentrating the light, and thus enable higher power objectives to be used.

Abbe condenser. The purpose of the condenser is to collect a large number of rays of light and bring them to a focus in the plane of the object to be examined. The plane mirror should always be used with natural light; the concave mirror with artificial light. The condenser may be focused by means of the screw beneath the stage of the microscope.

Diaphragm. The purpose of the diaphragm is to regulate the amount of light reflected by the mirror through the condenser. For stained preparations of bacteria the iris diaphragm must be open. In the examination of unstained and living forms the light should be diminished by partial closure of the diaphragm, so as to emphasize the slight variations in density of the protoplasm.

light. The mirror should be so adjusted as to illuminate the field eveuly when viewed through the tube of the microscope with the ocular removed. North light is to be preferred, and direct sunlight should never be used. The light coming from a white cloud or the clear sky is best.

Focusing. It should be a rule to work with as low a power as possible, so as to cover as large a field as is permissible. Always begin the examination of preparations of bacteria with a 4-mm. objective and a 2-inch ocular, using the 2-mm. oil-immersion objective for a more detailed examination.

The proper objective should be turned into place, and with the eye held at the side of the stage, the tube should be lowered carefully by the use of the coarse adjustment until the lens is nearly in contact with the cover glass of the preparation. With the eye at the ocular, then focus slowly upward with the fine adjustment, meanwhile moving the preparation 
slightly with the fingers. With high-power lenses never focus down with the eye at the ocular.

Oil-immersion objective. The rays of light passing obliquely through glass into air are refracted away from the perpendicular; or, in other words, the rays of light entering the lens of the microscope are less in number than if there were no difference between the indices of refraction of glass and

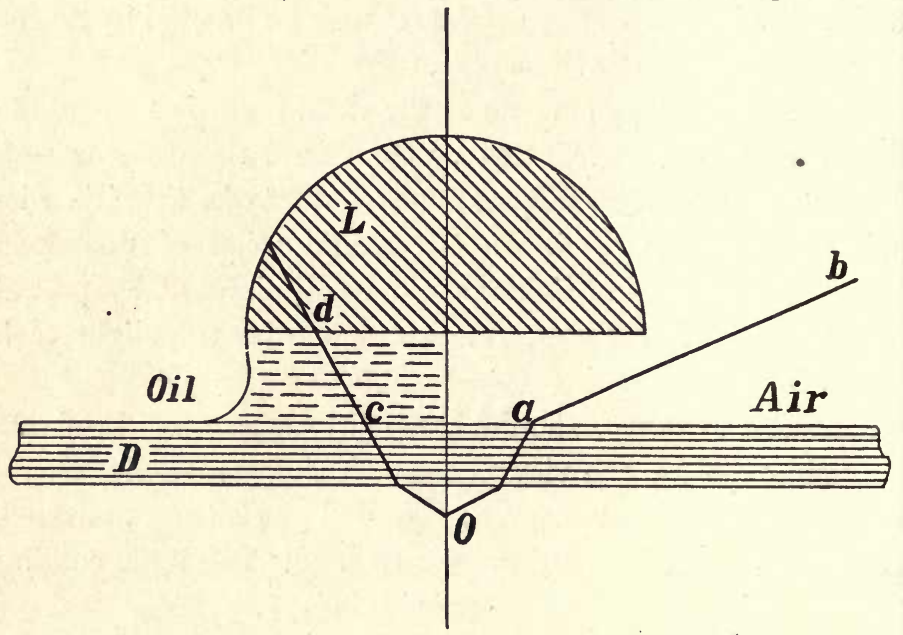

Fig. 10. Principle of the Oil-Immersion Lens

$L$, lens; $D$, object slide; $O d$ and $O b$, rays of light. (After Hager-Mez)

air. By placing a liquid having the same index of refraction as glass between the lens and the object, the amount of light entering the lens is increased, and as far as the amount of light is concerned, the object, the slide, the lens, and the intervening liquid act as a single piece of glass. The immersion liquid used is cedar oil, of such a degree of concentration as to have the same index of refraction as glass. If the oil becomes thickened by evaporation of the solvent, the bottle should be cleaned and a fresh supply obtained. 
In using the immersion lens a drop of cedar oil is placed on the cover glass, care being taken to avoid air bubbles. The tube of the microscope is lowered until the lens touches the drop and is nearly in contact with the cover glass. This must be done with care, for if the tube is lowered by the coarse adjustment until the objective touches the cover glass, a broken preparation or a damaged lens is likely to result. In some of the modern types of microscopes the fine adjustment is so arranged that its action is stopped when the objective touches the preparation.

At the end of the day's work the oil must be removed from the lens by wiping with Japanese lens paper, which should be kept in an envelope or glass jar to protect it from dust. If the lens becomes sticky by the drying of the oil, the latter must be removed by saturating a piece of lens paper with xylene and wiping the lens carefully. An excess of xylene must be avoided, as it is a solvent of the lens mounting. After the treatment the lens should be dried with clean paper.

Slides and cover glasses. The American slide is $25 \times$ $75 \mathrm{~mm}$. of clear white glass. The cover glasses used for bacteriological work must be thin on account of the short working distance of the higher-power objectives. No. 2 cover glasses are usually employed, having a thickness of $0.17-$ $0.25 \mathrm{~mm}$. The slides may be cleaned by washing in water or alcohol and drying with a towel. The cover glasses must be wholly free from fat, in order that an even distribution of the liquid to be examined may be obtained. New cover glasses are cleaned by washing in water, and then immersing in alcohol to which 3 per cent of hydrochloric acid has been added. The cover glasses are dried by rubbing between driers made of wooden blocks $200 \times 100 \times 25 \mathrm{~mm}$., covered with several 
layers of cotton cloth. The glass covers are then placed on a flat piece of Russia iron and heated over a Bunsen flame for one hour. The iron should not become red-hot. The prolonged heating destroys all traces of fat. The clean covers should be handled with forceps, for if touched with the fingers a film of grease is left. If a drop of water is placed on a perfectly clean cover glass, it will spread in a film over the entire surface. Store the cover glasses in a clean Petri dish.

Slides and covers that have been used may be cleaned by placing them in turpentine to remove the oil, then in a cleaning fluid composed of 20 grams of potassium bichromate, $100 \mathrm{cc}$. of water, $100 \mathrm{cc}$. of sulphuric acid. They should be digested in this mixture until the organic matter is destroyed. Rinse until free from the cleaning mixture, and then treat as new. The slides and covers may also be cleaned by placing in alcohol and adding 2-3 volumes of concentrated nitric acid, allowing them to stand in this for some time, rinsing, rubbing to remove any organic matter not destroyed, and wiping from alcohol. The addition of the acid should be made out of doors or in a hood, on account of the fumes given off.

Exercise. Clean $\frac{1}{2}$ ounce of cover glasses and 50 slides.

Staining solutions. The size and transparency of the bacteria make their differential study difficult in unstained preparations. In order that their morphology may be more readily determined, the use of stains or dyes is resorted to. The stains usually employed are the basic aniline dyes. For ordinary purposes methylene blue, fuchsin, gentian violet, and bismarck brown are sufficient.

The stock solutions kept in the laboratory are made by preparing a saturated solution of the various dyes in 95 per 
cent alcohol. These stock solutions keep well, but cannot be used for staining purposes. From them the solution used is prepared by dilution with distilled water, and filtering the mixture.

1. Aqueous solution of fuchsin is made by adding to $95 \mathrm{cc}$. of distilled water $5 \mathrm{cc}$. of the saturated alcoholic solution.

2. Aqueous solution of methylene blue is similarly prepared.

3. Aqueous solution of gentian violet is similarly prepared.

4. Lœffler's, or alkaline methylene blue, is made by mixing 30 cc. of saturated alcoholic solution, 1 cc. of a 1 per cent potassium hydrate solution, and $100 \mathrm{cc}$. of distilled water.

5. Carbol-fuchsin is made by adding to a 5 per cent solution of carbolic acid a saturated alcoholic solution of fuchsin until a metallic luster is noted on the surface of the liquid.

6. Aniline-water gentian violet is prepared as follows: To $98 \mathrm{cc}$. of distilled water are added $2 \mathrm{cc}$. of aniline oil. Shake vigorously. Filter, and to $75 \mathrm{cc}$. add $25 \mathrm{cc}$. of saturated alccholic solution of gentian violet. The solution should be refiltered after standing twenty-four hours, to remove drops of oil that may have settled out.

7. Iodine solution (Lugol's or Gram's solution) is prepared as follows: One gram of iodine is dissolved in a solution made of 2 grams of potassium iodide in $300 \mathrm{cc}$. of distilled water.

These staining solutions keep well with the exception of aniline-water gentian violet, which must be renewed at frequent intervals.

Exercise. From the saturated alcoholic solutions furnished, prepare $50 \mathrm{cc}$. of an aqueous solution of methylene blue, gentian violet, aniline-water gentian violet, carbol-fuchsin, and iodine solution. Place them in dropping bottles, or in bottles provided with corks through which a piece of small glass tubing is passed, by which small quautities of the solution can be removed without spilling. 
Making of stained preparations. Most forms of bacteria found in milk and its products can be stained by the use of the simple aqueous stains. The staining is carried out as follows: A clean cover glass is removed from the dish in which they are stored, and placed in a pair of cover-glass forceps. On the cover is placed a very small drop (size of a pin head) of water by means of the platinum loop, or from the glass tip of the flushing arrangement. With a sterile needle a minute portion of the growth on a solid medium is removed, mixed with the drop of water, and spread over as large a portion of the cover glass as possible. When liquid cultures are to be examined, a loopful should be removed and spread over the cover glass. The preparation should be allowed to air dry. If subjected to the action of heat, the individual organisms are heaped up in concentric masses, instead of being uniformly distributed. The preparation may be made on the slide itself, thus eliminating the use of the cover glass.

In order to cause the bacteria to adhere to the glass and not be washed off in subsequent operations, the preparation must be fixed by passing it rapidly through the upper part of a Bunsen flame three times (film side up). With nearly all forms this is sufficient to cause the coagulation of the proteids of the cell and thus permit of adhesion to the glass. When difficulty is experienced in fixing the preparations in this way, a couple of drops of 95 per cent alcohol may be allowed to evaporate from the preparation during the ordinary fixing process.

The forceps with the cover glass or the slide are placed on the staining dish and the preparation flooded with the stain, which is allowed to act from one to five minutes, after which it is washed off with a stream of distilled water. The wet cover glass is taken in a pair of fine-pointed forceps and 
placed film side down on a clean slide, avoiding air bubbles by allowing first one side of the cover glass to come in contact with the slide, and then gradually lowering the cover glass into place. The excess of water is removed and the upper surface of the cover glass dried by blotting with filter paper. The preparation is now ready to be examined directly, or a permanent preparation may be made by allowing the

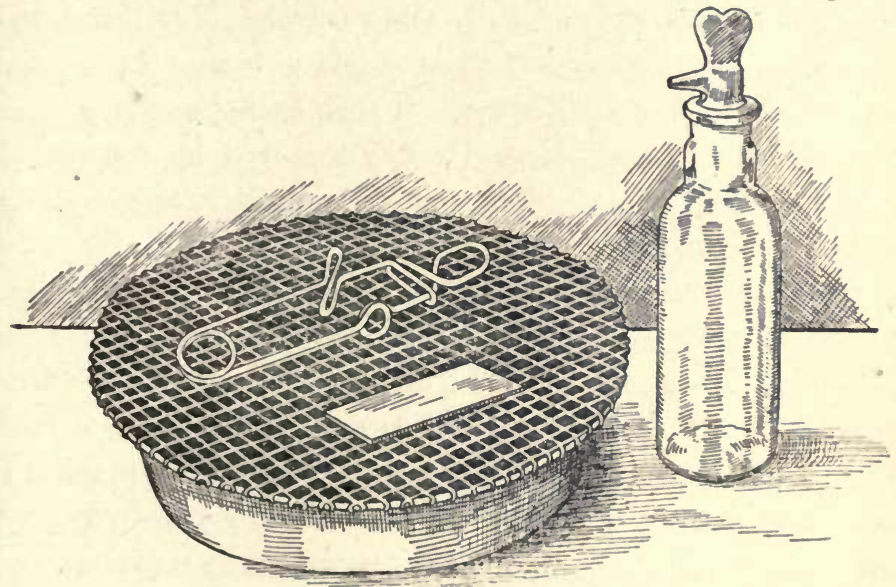

Fig. 11. Stainivg Dish axd Dropping Bottle for Stains

An enameled-ware basin covered with 1-inch-mesh galvanized wire cloth

cover glass to air dry after staining and washing. On a slide place a small drop of Canada balsam dissolved in xylene, and then lower the cover-glass preparation, film side down, on to the drop. The balsam should be of such consistency that it will spread under the weight of the cover glass, and should be present in such quantities that it will fill the space between the cover glass and slide to the edge of the cover, but not run beyond. The preferable method is to make a preliminary examination of the preparation in the water 
mount. If it is then desired to make a permanent mount, allow the preparation to dry, when the cover will fall from the slide when the latter is gently tapped. The cover can then be mounted in balsam. Allow the balsam to become dry before examining again (which process takes several days), as otherwise the cover glass is likely to be displaced. Preparations made on the slide may be drained, carefully blotted with filter paper, and allowed to become perfectly dry before examining, as the immersion oil is placed directly on the preparation. If it is desired to make a permanent mount of such a preparation, the immersion oil is removed by the use of xylene, a drop of balsam placed on the slide, and a cover glass placed in position.

When a preparation is properly made from a surface growth on solid media, the bacteria should appear as though suispended in air, i.e. the field of the microscope should be perfectly clear and the bacteria evenly distributed in the field. The common error is the use of too much growth, resulting in a thick film in which the bacteria are crowded together and piled up in such a manner as to make a satisfactory study impossible.

Exercise. Make cover-glass preparations from the cultures furnished you, using methylene blue or alkaline methylene blue for preparations from milk, broth, and gelatin cultures, gentian violet for preparations from agar.

Examination of stained preparations. In the examination of cover-glass preparations the following points should be noted: (1) the form of the bacteria, whether spherical, elongated, or spiral; (2) the grouping of the cells, whether isolated, in long or short filaments, spherical cells in plane surfaces, in irregular masses, or in packets; (3) the internal structure of the cell, in both faintly and deeply stained 
preparations, the presence of vacuoles, capsules, spores, the ease with which the bacteria take the simple aqueous stains.

Gram's stain. Some stains are of value in that they not only bring out the morphology of the organism, but also have a diagnostic value in differentiating one species from another. The one most frequently used for this purpose is the Gram stain. The staining of the preparation is as follows: Prepare and fix the preparation as previously described, and then stain for one minute with aniline-water gentian violet. Wash the preparation and then flood with iodine solution, leaving it in contact with the preparation for two minutes. Flood with 95 per cent alcohol, repeating this operation until no more color is given off. Wash, mount in water, and examine. Organisms which retain the stain are said to be Gram positive, all others Gram negative.

Exercise. Stain preparations from the cultures furnished by Gram's method.

Flagella stain. The staining of the delicate flagella is a difficult process. No one method is always successful. The number and arrangement of the flagella are of diagnostic value. The following method (Bunge's) is one of the best in use: A mordant, which so alters the protoplasm of the cell as to make it more readily stained, is prepared by mixing 25 cc. of a 5 per cent aqueous solution of ferric chloride with 75 cc. of a saturated aqueous solution of tannic acid.

From an eighteen-hour to a twenty-four-hour culture of the organism on agar remove a small amount of the growth and place in a large drop of tap water. Do not mix, but allow the organisms to diffuse themselves, as mixing with the needle breaks off many of the flagella. After standing a few moments remove a loopful to a perfectly clean cover glass and allow the drop to spread of itself. Dry, and fix 
with extreme care, to avoid overheating, by holding the cover glass in the fingers and passing through the flane. Flood the cover glass with the mordant and heat over the steam from a water bath for two minutes. Wash off the mordant and stain for two minutes with carbol-fuchsin, placing the cover over the steam. Wash, dry, and mount in balsam. The number and location of the flagella should be noted.

Exercise. From the cultures furnished make stained preparations. Determine the presence or absence of flagella.

Capsule stain. In certain culture fluids containing albumen or sugar many organisms produce a more or less thickened cell wall. The inner side of the cell wall appears as a sharp line, while the outer is less sharp and at times is not to be distinguished. The cell wall absorbs a large amount of water and forms a jellylike substance. In the stained preparation the cell contents appear deeply stained, the wall and swollen outer layer very faintly stained, or unstained. The organisms appear as though surrounded by a clear zone,- the capsule. Such organisms are of importance in dairy bacteriology, since they are frequently one of the causes of an abnormal fermentation of milk and cream, the so-called "slimy" or "ropy" fermentation.

A special stain may be used to determine the presence of the capsule. Prepare the film without the use of water, air dry, fix, apply glacial acetic acid, and drain off at once; without washing, apply carbol-fuchsin, renewing several times to remove the acid; wash in a 1-2 per cent salt solution; examine in salt solution. The glacial acetic acid fixes the mucilaginous capsule so that it does not dissolve in water.

Exercise. Examine by the above method the cultures furnished you.

Spore stain. The presence or absence of spores cannot be determined by staining in all cases. Vacuoles, fat drops, 
crystals, may be mistaken for spores in preparations stained with simple aqueous dyes. A special stain may be employed as follows: Place the dried and fixed films in chloroform for two minutes, and then in a 5 per cent solution of chromic acid for two minutes; wash in water, cover with carbol-fuchsin, and heat in the steam of a water bath for five minutes. Wash off the stain; decolorize carefully in 1 per cent sulphuric acid; wash in water; counterstain with methylene blue at room temperature for ten seconds; wash and examine.

The principle of the process rests on the peculiar property of the spores towards stains. They stain with difficulty, but, when once stained, retain the color. Thus the first stain can be removed from the cell with a weak acid, and the cell then counterstained with a differential dye. The position of the spores in the cells should be noted, whether medium or terminal ; their shape, whether oval or round ; their size in relation to the mother cell, whether greater in diameter than the mother cell, or of less diameter; the presence of free spores, i.e. outside of the mother cell.

The only method by which the spores may be identified as such in case of doubt is by observing their germination. This also is often of diagnostic value, and can be observed in the hanging block suggested by Hill.

Exercise. Make spore stains from cultures furnished you.

Examination of living bacteria. The examination of the bacteria in a living condition will give much information of morphological value. The method of procedure is as follows: Place a drop of liquid medium - broth - on a cover glass or slide, and inoculate it with a particle of growth from a solid culture, mix carefully, and transfer a very small drop to a cover glass; do not spread. Invert over the drop a hollow ground slide around the cavity of which has been placed a 
ring of vaseline; press down on the cover. The vaseline should form a perfect seal, thus preventing evaporation and enabling a small drop to be kept under observation for hours. On account of the transparency of the bacteria focusing is often difficult. The drop should be placed in the optical axis of the microscope, which is easily done by lowering the tube with the 4-mm. objective in place within one eighth of an inch of the cover glass, and then bringing the drop under the lens. With the eye at the side, lower the tube until the objective is nearly in contact with the cover. Close the diaphragm until the field is light gray in color, and focus very slowly upward. After finding the organisms the slide should be moved until

Fig. 12. A Hanging-Drop Preparation

the edge of the drop is found, as here the organisms can be observed to better advantage than in the deeper portion of the drop. The oil-immersion lens may then be used if desirable. The use of artificial light renders the organisms more easily visible.

The form, size, and grouping of the cells should be noted, the power of independent motion, the nature of the motion, - direct, sinuous, rotating, etc. The molecular or Brownian motion (pedesis) must not be confounded with vital motion. This molecular motion is a property common to all finely divided substances suspended in liquids, and consists of a vibratory or trembling rather than a progressive motion. In order that an organism be classed as motile, the relative positions of the cell with reference to other cells must change.

Exercise. Examine in hanging drop the cultures of yeast and of bacteria furnished you, and also a preparation made by suspending a little India ink in water. 
Hanging block. It is frequently desirable to determine the mode of germination of bacterial spores, and the mode and rate of fission of the vegetating cell. This is most conveniently done by the use of Hill's hanging block. Pour melted nutrient agar into a Petri dish to the depth of one eighth of an inch. Cool the agar. Cut from it with a sterile knife a block one quarter to one third of an inch square, and of the thickness of the agar in the dish. Place the block, under surface down, on a glass slide, and protect from dust. Prepare in sterile water a suspension of the organism to be examined, if it has been grown on a solid medium, or, if a broth culture is used, spread the suspension on the upper surface of the block as though making a cover-glass smear. Place the slide with the block in the $37^{\circ} \mathrm{C}$. incubator for five or ten minutes to dry. Then lay a clean sterile cover glass on the inoculated surface of the block in close contact with it, avoiding air bubbles as far as possible. Remove the block from the slide and invert the same. With a platinum loop run a few drops of melted agar around the edge of the block, to fill the angles between the sides of the block and the glass. This seal prevents slipping of the block. The block should again be placed in the incubator for five to ten minutes to dry. Invert this preparation over a moist chamber made by cementing a ring of glass to an ordinary slide. The hollow ground slide cannot be used on account of the depth of the block. The cover should be sealed in place with white wax or paraffin. Vaseline softens at $37^{\circ} \mathrm{C}$. and allows the cover glass to shift. The preparation may now be observed with the $\frac{1}{12}$-inch immersion objective.

Measuring bacteria. Measurements under the compound microscope are made by means of an ocular micrometer, - a disk of glass which is placed on the diaphragm within the 
ocular. The glass bears a scale made up of lines at an equal but not necessarily known distance apart. The scale of the micrometer should lie in the plane of the real image formed by the objective. The image of the object thus lies on the scale, and may be measured by it if the value of each division of the scale be determined in actual units of measurement. For this purpose a stage micrometer is necessary, - a slide bearing a cover glass on which is ruled a scale in 0.1 and $0.01 \mathrm{~mm}$. The ratio of ocular divisions to the image of the stage divisions is determined by focusing with the ocular micrometer in place on the scale of the stage micrometer. The lines of the two micrometers are made parallel by rotating the ocular. Make the image of any two lines on the stage micrometer coincide with two lines on the ocular micrometer, by drawing out the tube of the microscope if necessary. From the data thus obtained it is easy to calculate the value of each space on the ocular micrometer in 0.01 of a millimeter. The ocular used, the objective, the number of the ocular micrometer, and the tube length of the microscope must be recorded and also the value of a single space on the ocular micrometer for the given system of lenses in microns (0.001 mm.) expressed by the Greek letter $\mu$.

Exercise. Determine the value of the ocular micrometer and fill out the blanks in the following table:

No. of microscope

Make

\begin{tabular}{c|c|c}
\hline ObJEctive & $\begin{array}{c}\text { VAlue of A Division } \\
\text { OF EYepiece Microniter }\end{array}$ \\
\hline $16 \mathrm{~mm}$. & & \\
\hline $4 \mathrm{~mm}$. & \\
\hline $2 \mathrm{~mm}$. & \\
\hline
\end{tabular}




\section{CHAPTER III}

\section{CONTAMINATION OF MILK}

Milk, meats, eggs, fruits, etc., are classed as perishable food products, for unless extraordinary means are taken to preserve them, the period during which they can be used as food is limited. Of these milk is the most perishable. The period during which it can be kept is measured by hours rather than by days. The spoiling of these products is due to the growth of bacteria and other organisms in and on the surface. The more perfectly the product is adapted as food for organisms, and the greater the number which gain entrance to it, the shorter will be its period of usefulness. No other food product is to be compared with milk as a culture medium for bacteria, and, curiously enough, no other food product is so exposed to contamination during the process of preparation.

No subject in dairy bacteriology is more fundamentally important than that relating to milk contamination, both in the factory and on the farm, and most of the problems relating to the proper handling of milk come back to this question.

The contamination of milk may be considered from three standpoints, - hygienic, economic, and what may be called the standpoint of general cleanliness. From the hygienic standpoint are to be considered the contamination with pathogenic bacteria and the production of poisonous and injurious substances in the milk by the growth of saprophytic bacteria. The economic side of the question has to do with the diminished keeping quality of milk and of other dairy products, 
and is especially important to the milk producer and handler, and also to the consumer. If in any practical way the contamination may be lessened and the keeping quality improved, the value of the product will be enhanced. Entirely apart from the economic and hygienic relations of the contamination of milk may be considered its relation to general cleanliness. Wholesome food prepared in a cleanly manner is desirable, not because unclean food may cause sickness or may not keep so long, but simply for the sake of cleanliness alone.

When it is considered that milk is largely produced for human food, either in the form of milk, or as butter or cheese, it must be admitted that the circumstances surrounding its production are, even under the best of stable conditions, far from what would be looked upon as desirable in the preparation of any other food product. Especially is this true when it is kept in mind that meat, fruits, vegetables, etc., $\rightarrow$ food products likely to be soiled with dirt, - can be washed and rendered clean and wholesome. The mud, manure, and dust which fall into milk are partially dissolved and cannot be removed. Straining removes the grosser pollution, such as straw and hair, but does not remove the bacteria which are attached to these objects. Milk is a food product that, once rendered dirty, cannot be made clean. Many of the sources of contamination are wholly preventable, some partially, and still others to a very slight extent. The student should become thoroughly familiar with all the sources of contamination and with the different practical means for overcoming or removing the same.

Contamination from barn air. Dust particles floating in the air invariably carry a larger or smaller number of bacteria, depending upon the nature of the surface from which 
the dust came. In the cow stable many operations are carried on that tend to raise a large amount of dust, which in many cases comes from materials that are very rich in bacterial life. The dried manure which is scattered over the floor, and in time pulverized, is an important source. The dust raised whenever cattle are brushed is largely made up of manure particles, epidermal scales, and scurf from the skin. The operation of feeding dry feed (hay, corn, fodder, etc.) is a source of dust, for the way in which these materials are harvested tends to contaminate them with soil from the fields, and the surface layers of the soil are especially rich in bacterial life. The dust raised during the various barn operations is comparatively heavy, and for the most part soon settles, carrying its load of germ life.

The relative extent and importance of this source of contamination under varying conditions may be determined as follows: Tubes of lactose gelatin are melted, poured into sterile Petri dishes, and allowed to become completely solidified. A number of these plates are exposed in the stable when dust is present in minimum quantities, i.e. at a time when no operation that produces dust has been carried on for two or three hours. The remaining plates should be exposed shortly after some dust-producing operation has been completed. This exposure should be made by placing the plates on a level surface, removing the cover, which is placed right side up by the side of the plate, so as not to add a further contamination when it is replaced. The exposures should be made in different parts of the stable and the plates so located as to be fully exposed to the falling dust, and yet be protected from dirt being thrown on to them. A definite period of exposure should be made, although this time may vary within reasonable limits (thirty to one hundred and twenty seconds). 
The plates should be examined daily and the final count made after from five to six days' incubation. Often the presence of a large number of molds and liquefying bacteria necessitates a much shorter period of incubation. The results may be recorded in terms of the number of colonies per plate, but for more graphic presentation should be converted into the number of organisms that would fall into a 12 -inch milk pail during an average milking period (five minutes).

Exercise. Each student will prepare ten plates as described : (1) expose four of them in the stable at a time when dust is present in minimum quantities; (2) four when different barn operations producing dust are in progress ; (3) the remaining plates are to be exposed in the immediate neighborhood of an animal that is being brushed.

Contamination from coat of animal. The udder and flanks of animals, even under the best of stable conditions, are likely to become soiled with manure, and unless elaborate precautions are taken to remove the dirt, it is certain to be an important source of bacteria in milk, as during the milking process the movements of the animal and milker will dislodge dust and accompanying bacteria not only from the udder proper but from the flanks and sides of the cow. More or less of this dirt and dust finds its way into the open milk pail.

The bacterial content of fresh manure is exceedingly high; hence very small quantities may increase the germ content of the milk to a marked degree.

The importance of these sources of contamination under various conditions may be determined as follows: Plates are prepared as in the previous exercise, and exposed under the udder of a cow while this organ is being manipulated as during the milking process. The culture plate should be exposed by placing it in a milk pail which is held in the same 
position as during milking. Wetting the inner surface of the pail prevents contamination from it. The exposure should not be continued for more than thirty seconds. The results should be finally expressed in numbers of bacteria that would fall into a 12 -inch pail under the conditions obtaining in the trial during the average time of milking (five minutes).

Animals in varying degrees of cleanliness should be selected. Exposures should also be made in a similar manner under the same animals immediately after their flanks and udder have been thoroughly moistened with a clean damp cloth.

Exercise. Each student will prepare four plates of lactose gelatin and allow them to solidify : (1) expose two plates for thirty seconds, as described, under animals which have not been previously treated; (2) expose the remaining two under animals whose udders and flanks have been thoroughly moistened with a clean damp cloth.

Contamination from manure. The amount of contamination which the milk receives from animals whose udders and flanks are soiled with manure or mud is not determined with any degree of accuracy by the exposure of plates in the manner described, since each particle of dirt may cause the development of a colony, although it may have carried hundreds of bacteria which would, in large part, be scattered through the milk, on account of the solubility of the manure. To gain a more correct idea of the extent of contamination that may come from this source, a quantitative determination of the bacteria in manure should be made by collecting some, if possible, from the flanks of animals. One gram should be weighed out on a piece of sterile filter paper. Place this in a definite amount of sterile water and shake until the manure is disintegrated and dissolved as completely as possible. Prepare plates with varying dilutions, as in the quantitative analysis of milk. 
Exercise. Each student will make a quantitative examination of fresh manure and also of dried manure removed from the flanks of cows.

A piece of sterile filter paper 4 inches square is placed on the scale pan, and on this a smaller piece. One gram of manure is weighed out on the sterile filter paper; the upper paper and manure are transferred to a flask of sterile water (500 cc.). Make as even a suspension as possible. The dilutions should be very large, especially in case of the fresh manure, $-1 / 10,000,1 / 100,000$, and $1 / 1,000,000$ grams, for example.

Plain agar medium should be used and the plates incubated for forty-eight hours at $37^{\circ} \mathrm{C}$, , or at $20^{\circ} \mathrm{C}$. for four to five days.

Express the results as the number per gram of sample examined.

Contamination from hair. Hairs removed from the flanks and udders of even the cleanest animal bear more or less dust and bacteria. The extent of this contamination can be measured by removing, with a pair of scissors, some of the long hairs from the udders and flanks and placing them in sterile Petri dishes for transportation to the laboratory. Lactosegelatin plates are prepared as previously. When the plates are well solidified three or four hairs are placed on the surface of the medium by means of a pair of forceps or the platinum loop. The hair should be pressed down so as to be thoroughly in contact with the medium. A separate culture dish should be used for the hairs from different animals. The results should be expressed as the average number of bacteria per linear inch on the hairs removed from the various animals.

Exercise. Each student will collect several individual hairs from four animals showing varying degrees of cleanliness, and determine the number of organisms on the same, noting the relative number of molds, liquefying and nonliquefying bacteria.

Contamination from interior of udder. Bacteria may gain entrance to the interior of the udder and establish themselves 
in the secreting portion of the gland and in the milk ducts and cistern. The portals of entrance are two. When pathogenic organisms are present in the circulating blood, they may pass into the udder and possibly into the milk ducts (foot-and-mouth disease). The more frequent manner is for the organisms to lodge in the tissues of the udder, where they grow, and from which they may be discharged into the milk ducts by the breaking of the abscess (inflammation of the udder, or tuberculosis).

The second portal of entrance is through the exterior openings of the teats. For a short time after milking the end of the teat is moist, and in the case of animals that leak the milk, constantly so. Bacteria collect at this point and gain their way into the teat, milk cistern, and milk ducts. Most of the forms that enter in this way do not find favorable conditions for growth, and soon disappear. Others are able to grow to some extent, and may penetrate to the secreting tissues of the gland. As a rule, no pathological disturbance follows the entrance of the bacteria, as most are saprophytic forms. At times cases of udder inflammation are produced by organisms entering through the teat. Contagious garget is spread by the milker in this way.

In order to determine the importance of this source of bacteria, samples of milk should be collected in such a manner as to exclude other sources of cortamination. The samples should be collected at different times during the process of milking. The results obtained will give an idea of the portion of the gland in which the largest number of bacteria are found. If the first few streams drawn from a quarter contain many more bacteria than the milk collected at the end of the milking process, then it is evident that the bacteria were largely in the teat and milk cistern. 
A sample of the foremilk, the first 10-15 cc. of a single quarter, should be drawn directly into a sterile, wide-mouthed bottle or flask, as well as a sample of the strippings from the same quarter. A sample should also be taken from the pail after the milking of the individual animal is completed. Quantitative analyses are to be made in the usual way. The various samples should be kept at room temperature and frequent observations made, in order to determine the keeping quality and the nature and flavor of the curdled milk.

Exercise. Each student will collect samples of foremilk and strippings from one quarter of each of two cows, and also from the pail at the end of the milking of each animal, and examine the samples quantitatively.

Contamination from utensils. The amount of contamination which the milk receives from the various utensils used in the handling depends entirely upon the cleanliness of the same. The degree of cleanliness of these utensils turns not only on the manner in which they have been washed, but upon the construction and condition of the utensil. A milk pail pressed from a single sheet of metal, and well tinned, is easily cleaned, for it has no crevices and joints into which the milk can penetrate, and from which it is impossible to remove it. A pail constructed in the ordinary way, with folded seams, is less easy to keep in a condition of bacteriological cleanliness. With the increased complexity of construction it becomes more and more difficult to maintain any utensil in a sanitary condition.

On the farm most of the utensils are of such a nature as to be cleaned easily, but in the creamery, cheese factory, etc., where cream separators, pasteurizers, and numerous pipes are made use of in handling the milk, the difficulty is increased. On the farm the most difficult utensils to keep clean are the 
farm separator and the milking machine, where this is in use. On many farms the separators are not taken apart for washing after each period of use, but the apparatus is rinsed out with water, either hot or cold, and left in an assembled condition. Sometimes the machine is taken apart and thoroughly cleaned only at considerable intervals, such as once a week, reliance for cleanliness being placed on the use of a solution of washing powder and subsequent rinsing with water.

The long rubber tubes which are necessary with the milking machine cannot be cleaned perfectly, and must be kept in an antiseptic liquid in order to prevent bacterial growth. For this purpose limewater or a weak solution of formalin is preferably used, although brine solutions have sometimes been recommended.

The number of bacteria adhering to any utensil, will depend upon the condition of the surface. If it is smooth, as in a new, well-tinned pail, the number will be much less than if the surface is roughened by rust. The difficulty in rinsing the milk from even a perfectly smooth surface can be illustrated by filling a pipette with whole milk and then trying to remove all visible traces of the milk by passing a stream of water through it.

If the milk is allowed to dry on a utensil, it is impossible to remove it by ordinary means, since the casein when dried forms a glue-like substance, which soften's in water very slowly and can be removed only by scouring.

Cloth strainers are likely to add more bacteria to the milk than they remove, unless they are so treated as to prevent all growth in the cloth. A strainer well rinsed in cold water, then in boiling water, wrung dry, and hung in the sun, or where it will dry rapidly, will contain small numbers of bacteria. A strainer simply rinsed in cold or lukewarm water 
and hung in a damp milk room or creamery will soon be a source of contamination.

The number of bacteria to be found in any utensil is dependent not only on the manner of washing, but on whether or not it has been thoroughly dried. If small amounts of water are left in cans, pails, bottles, etc., a profuse growth of bacteria will take place, for the water will contain sufficient organic matter for food, no matter how carefully the utensil was cleaned.

The bacteriological condition of any utensil can be determined by rinsing it with sterile water and preparing quantitative plates from the water. The walls and bottoms of pails, cans, etc., should be scrubbed with a sterile brush, especial attention being given to the seams. Cloth strainers may be rinsed in sterile water and this examined. Milking machines should have a considerable volume of water passed through the teat cups and rubber tubes into the can. Separators are examined in a similar way.

Exercise. Each student will test the bacteriological condition of pails, strainers, and farm separators, which have been treated in the various ways generally employed in farm and creamery practice.

PaIls and Cans. Sterilize two flasks of water containing $100 \mathrm{cc}$. each. Place two test-tube brushes in large test tubes, stopper with cotton, and sterilize with the flask of water. Pour a flask of the sterile water into the utensil to be examined, scrub the walls and bottom, and especially the seams with the test-tube brush. Return the water to the flask and from it prepare quantitative lactose-agar plates. From the data obtained, calculate the number of bacteria that would have been added to the milk in case the vessel used had been filled with inilk.

Cloth strainers. Sterilize a liter of water in a small pail or can. Rinse a strainer in this water as well as possible, wringing out the cloth. Examine the water quantitatively.

Farm SeParators. A quantity of milk should be passed through the separator and the milk removed by flushing the machine with a couple of gallons of cold water. 
From twelve to twenty-four hours later pass through the machine a definite quantity of water which has been boiled for a few moments, in order to sterilize it, and then cooled. Catch samples of the water in sterile test tubes and examine quantitatively.

Repeat the trial by passing milk through a separator for four days, cleaning the separator after each day's use by flushing with cold water, then with a hot solution of washing powder, then with hot water, to remove the washing powder. At the end of the period pass sterile water through and examine as before.

Washing utensils. The student should study the influence which the manner of washing utensils has on their germ content.

Few farms are provided with facilities for production of steam, so it is much more difficult to clean utensils properly on the farm than in the factory.

Cans or pails should be washed in the same manner as on the farm, and the results obtained compared with those obtained from the examination of a pail or can washed in a similar way and then steamed.

Exercise. Each student will wash the cans furnished him in the following manner: Rinse with cold water, wash in a warm solution of washing powder, and rinse with scalding water. One of the cans should be examined as described in the previous exercise. The other should be steamed for thirty seconds over a steam jet and then examined.

Plan for the production of sanitary milk. Each student will prepare and submit for examination a plan for the establishment of a plant for the production of milk of high grade, and for the management of the plant. This plan should include everything which may have a direct or indirect relation to the milk as human food. The construction of the stable has an indirect relation to the contamination of the milk, the health of the animal to the hygienic requirements, etc. 


\section{CHAPTER IV}

\section{MILK FERMENTATIONS}

In the study of medical bacteriology the specifie causal organism of any disease is the important thing; hence great emphasis is laid on the study of the organism in question, its isolation and its identification. In dairy bacteriology the various types of fermentations are not produced by a single species, but rather by a group of organisms capable of causing, in a general way, similar chemical changes. The organisms connected with these changes may differ much among themselves. The ordinary souring of milk may be produced by a host of bacteria having not only dissimilar morphological characteristics but cultural ones as well; some of them are micrococci, others small bacilli, and yet others large rodshaped forms. In dairy bacteriology the character of the fermentation and the nature of by-products formed in milk are the important things. Hence the more detailed study of specific organisms is of somewhat less importance than in medical bacteriology.

It is, however, very desirable for the student to study representative organisms of each group, even though the specific forms are not identified, for work of this character is needed to teach the beginner the necessity of noting easily overlooked cultural differences that would not be observed except by close comparative study. In the detailed study of any organism certain points in regard to the morphology, cultural characteristics, and biochemical reactions must be 
determined. The important questions are those that admit of a positive or negative answer. Those which involve detailed descriptions are of less importance to the beginner; hence, with a few exceptions, not so much emphasis is laid on the cultural characteristics as on the morphology and the biochemistry of the organism.

Preliminary cultivation. Whenever an organism is to be studied and an attempt made to determine to what particular group it belongs, it is important that it be in a state of high vitality, as otherwise uniform results cannot be obtained. In nearly all cases the optimum conditions for the growth of an organism are not known. It has been determined that by a preliminary cultivation the constancy of the results obtained may be materially increased. The standard procedure of preliminary cultivation is as follows: From an agar culture of the organism a broth culture is made, and incubated for twenty-four hours at $20^{\circ} \mathrm{C}$. Transfer from this broth culture to a second tube of broth, and incubate for twenty-four hours at $20^{\circ} \mathrm{C}$; repeat a third time. In order to be certain that the final trials are made with a pure culture, from the third broth tube gelatin plates are prepared. From one of the colonies on the gelatin plates inoculate a tube of sloped agar and incubate for forty-eight hours at $20^{\circ} \mathrm{C}$. From this the subsequent inoculations are to be made into various media.

This method of restoring the vitality of the organism presupposes that it will find favorable conditions for growth on the ordinary nutrient media and at a temperature of $20^{\circ} \mathrm{C}$. With some groups of bacteria, important in the dairy, this method of preliminary cultivation should be modified. The great group of lactic-acid bacteria grow very poorly, often not at all, in the ordinary nutrient broth. If, however, this 
be qualified by the addition of a fermentable sugar, the organisms find much more favorable conditions for growth.

Primary tests for identification of species. The primary tests for the identification of bacterial species are those that can be answered positively or negatively. In the study of an organism the following scheme should be employed: The positive results should be expressed by a cross $(+)$, a double cross (\#) indicating an emphatically positive result; zero (0) indicates a negative result; a question mark (?), a doubtful result; and a blank space indicates that no determination was made.

\section{Primary Tests for Identification of}

1. Source Bacterial Species

2. Number or name

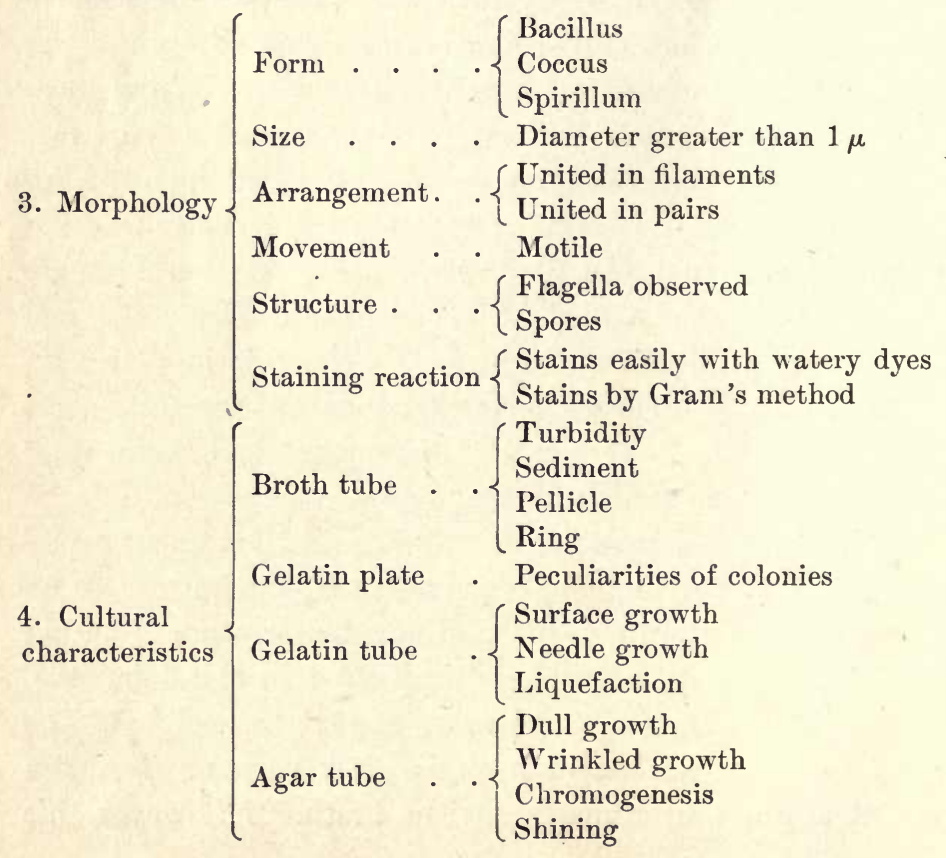




\begin{tabular}{|c|c|c|}
\hline \multirow{8}{*}{$\begin{array}{l}\text { 5. Biochemical } \\
\text { reactions }\end{array}$} & Milk . . . . & $\begin{array}{l}\text { Coagulation } \\
\text { Degree of reaction } \\
\text { Casein liquefaction }\end{array}$ \\
\hline & Dextrose broth & $\left\{\begin{array}{l}\text { Gas } \\
\text { Acid }\end{array}\right.$ \\
\hline & Lactose broth & $\left\{\begin{array}{l}\text { Gas } \\
\text { Acid }\end{array}\right.$ \\
\hline & Saccharose broth & $\begin{array}{l}\text { Gas } \\
\text { Acid }\end{array}$ \\
\hline & Nitrate broth & Nitrite formation \\
\hline & Peptone broth . & Indol production \\
\hline & $\begin{array}{c}\text { Relation to } \\
\text { oxygen }\end{array}$ & $\begin{array}{l}\text { Aërobic } \\
\text { Facultative anaërobic } \\
\text { Anaërobic }\end{array}$ \\
\hline & $\begin{array}{l}\text { Relation to tem- } \\
\text { perature }\end{array}$ & $\begin{array}{l}\text { Growth at } 20^{\circ} \mathrm{C} \text {. } \\
\text { Growth at } 37^{\circ} \mathrm{C} \text {. } \\
\text { nt below } 80^{\circ} \mathrm{C} .\end{array}$ \\
\hline
\end{tabular}

The morphological study should be made on fully developed cultures. Cultures which are growing rapidly may present a large number of immature forms. Old cultures may contain degenerated and altered forms (involution types). No definite statement can be made in regard to the age of a culture, but, as a rule, those grown at $37^{\circ} \mathrm{C}$. should not be over twenty-four to forty-eight hours old, and those grown at $20^{\circ} \mathrm{C}$. from forty-eight to seventy-two hours old. Fxaminations of material should be made from both solid and liquid media in both stained and unstained preparations.

In Appendix B is given the scheme for the detailed study of an organism, recommended by the Society of American Bacteriologists, together with a glossary of the terms employed in describing the cultural characteristics of an organism.

Action on carbohydrates. The ability of the bacteria to ferment sugar with the production of gas and acid is best determined by the use of the fermentation tube, since this 
procedure allows not only the qualitative determination to be made, but also permits of a rough quantitative analysis and the determination of the relative amounts of the component gases formed.

In case the fermentation tubes filled with sugar broth have not been recently sterilized, heat in the sterilizer in order to

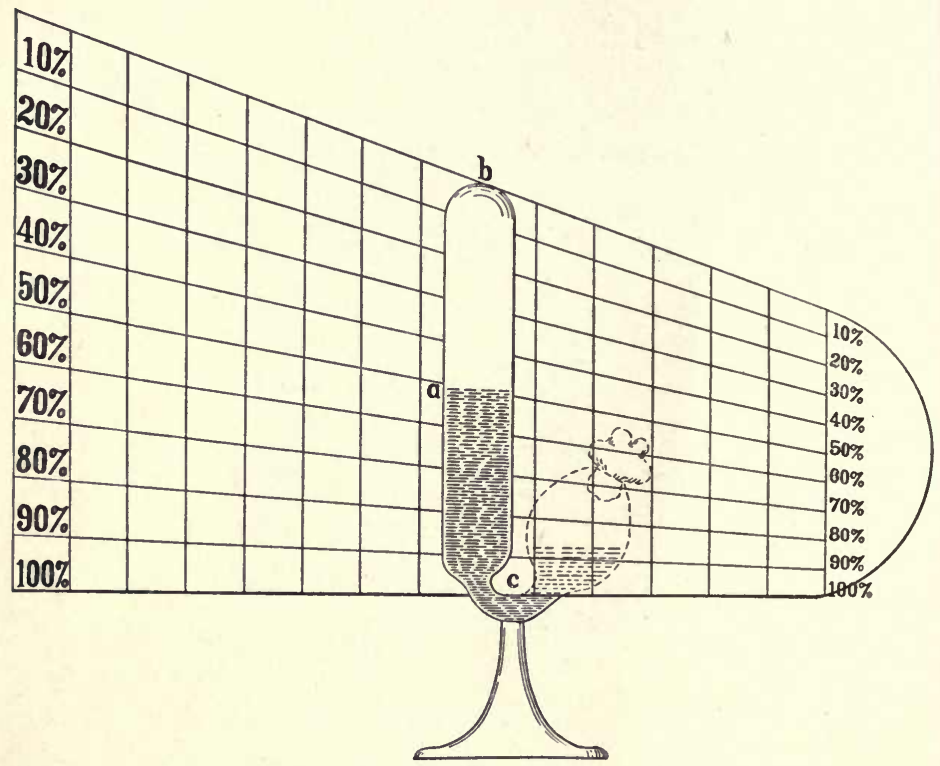

Fig. 13. Frost's Gasoneter

The amount of gas is expressed in per cent of the length of the closed arm of the fermentation tube. (After Heinemann)

drive off dissolved oxygen. If they contain bubbles of gas on being taken from the sterilizer, remove them by decantation. When ready to use, the liquid should fill the closed arm and stand at a low level in the open bulb. When cool, inoculate. At frequent intervals during the incubation, which, as a rule, should be made at $37^{\circ} \mathrm{C}$., the presence or absence of 
gas in the closed arm should be noted, and the amount of gas determined by measuring the same with Frost's gasometer.

When the culture ceases to form gas, remove from the incubator, cool to room temperature, and measure. Test the reaction of the liquid in the open arm by the use of litmus paper, or titrate a definite amount as in the case of milk. The culture should not be allowed to stand too long after gas evolution ceases, as the carbon dioxide formed is rapidly absorbed by the liquid.

To determine the relative proportion of the component gases formed, fill the bulb of the fermentation tube with a 2 per cent solution of sodium hydroxide, close the opening of the tube with the thumb, tip the tube so as to allow the gas to fill the bulb and come in contact with the alkaline solution; return the gas to the closed arm, and repeat the process several times, keeping the open arm tightly closed. With the gas in the closed arm, remove the thumb. The liquid will rise in the closed arm on account of the absorption of the $\mathrm{CO}_{2}$ by the alkali. Measure the residue, which is usually considered as hydrogen, close the tube again with the thumb, and bring the gas into the bulb; remove the thumb and introduce a lighted match. The air mixed with the hydrogen forms an explosive mixture. The composition of the gas is expressed by the ratio of hydrogen to carbon dioxide $\left(\mathrm{H} / \mathrm{CO}_{2}\right)$.

With bacteria that do not grow at $37^{\circ} \mathrm{C}$. the test should be made at $20^{\circ} \mathrm{C}$, the time of incubation being extended at least four days.

A qualitative test for gas production from the various sugars can be made by the use of "shake.cultures." A tube of melted gelatin or agar containing 2 per cent of the sugar to be tested is inoculated with the culture, mixed well, and solidified by placing in ice water. If the sugar is fermented 
by the organism with the production of gas, the medium will be rent by the gas. Tubes of broth can also be used for the qualitative determination of gas by placing in the tube before sterilization a small piece of glass tubing sealed at the upper end. In the sterilization the air is completely driven out and the small tube filled with the broth. If gas is formed in the developing culture, bubbles will collect in the small tube.

When it is desired to determine whether or not an organism is able to ferment a certain sugar, it is essential that the medium to which the sugar is added be free from sugar. In media prepared from meat or beef extract, a small amount of muscle sugar (inosite) is present. This can be removed by inoculating the meat infusion, after straining, with a culture of an organism able to ferment inosite (B. coli communis), and incubating for twelve to sixteen hours, or until all the sugar is decomposed. The medium is then finished in the usual manner.

In the testing of sugars easily affected by heat, it is essential that the sterilization be as short as possible, otherwise the sugar may be decomposed into its constituent sugars and these be then fermented. Saccharose in slightly acid solutions is easily inverted, lactose far less easily.

Exercise. Inoculate fermentation tubes filled with lactose broth from the cultures furnished. Determine the amount and composition of the gas produced (see p. 69). Also inoculate tubes of melted lactose agar from the various cultures.

Action on nitrates. Many organisms have the power of reducing nitrates with the formation of nitrites, and sometimes ammonia and free nitrogen. Nitrate broth is prepared by dissolving 1 gram of peptone in 1 liter of tap water and adding 0.2 grams of nitrite-free potassium nitrate. Tube and sterilize in the usual way. Inoculate and incubate for 
four days at $37^{\circ} \mathrm{C}$., together with an uninoculated tube to serve as a control.

Test for nitrites. Dissolve 8 grams of the purest sulphanilic acid in $1000 \mathrm{cc}$. of 30 per cent acetic acid (sp. gr. 1.041). Dissolve 5 grams of $a$-amidonaphthylamine in 1000 cc. of 30 per cent acetic acid; filter through a plug of washed absorbent cotton. At the end of the period of incubation in the nitrate broth remove $3 \mathrm{cc}$. of the culture to a clean test tube and add 2 drops each of the naphthylamine and sulphanilic acid solutions. The development of a red color indicates the presence of nitrites, the intensity of the shade indicating in a general way the amount of nitrites present. The control tube should be treated in the same manner as the culture.

Test for ammonia. Nessler solution. Dissolve 50 grams of potassium iodide in a minimum quantity of cold water. Add a saturated solution of mercuric chloride until a slight but permanent precipitate persists. Add 400 cc. of a 50 per cent solution of potassium hydrate made by dissolving the potassium hydrate and allowing it to clarify before using. Dilute to 1 liter and allow the mixture to settle. One half of the remaining portion of the culture used for the nitrite test is removed to a test tube and a couple of drops of the Nessler solution added. The presence of ammonia is indicated by a yellow color, or by a yellow precipitate when the ammonia is present in considerable quantities.

The presence of ammonia in broth culture may be detected by moistening a strip of filter paper with the Nessler solution and placing the same in the neck of the tube, a yellow to reddish-brown color indicating the presence of ammonia.

Exercise. From the cultures furnished, inoculate tubes of nitrate solution. Determine, as described, the ability of the organisms to produce nitrites and ammonia from the nitrate present. 
Formation of indol. Many bacteria produce a number of aromatic compounds, the most important of which is indol, one of the series associated with nitrogenous decomposition, as in fecal matter. Indol is produced only in the absence of sugar. Sugar-free broth must thus be used. The incubation of the meat infusion must be sufficiently prolonged to remove the sugar, but not so extended as to permit of indol formation. The use of peptone solution is preferable for this purpose ; to make this solution, 10 grams of peptone are dissolved in $1000 \mathrm{cc}$. of water, tubed, and sterilized.

The broth is inoculated and incubated for four days at $37^{\circ} \mathrm{C}$. To test for indol add $1 \mathrm{cc}$. of 0.01 per cent solution of sodium nitrite, mix, add a few drops of concentrated sulphuric acid, allowing it to flow down the side of the tube, forming a layer at the bottom. If indol is present, a pink or red ring forms at the junction of the two liquids, due to the formation of nitroso-indol. The tubes should be allowed to stand for half an hour before final judgment is recorded.

Exercise. Inoculate tubes of sugar-free broth from the cultures furnished. Test for indol as described.

Relation to oxygen. The relation of the bacteria to oxygen may be determined by noting the character of the growth in the fermentation tube. If the tubes are heated in the sterilizer shortly before inoculation, the oxygen dissolved in the medium will be driven off, and in the closed arm anaërobic conditions will prevail. If the developing growth is limited to the open arm of the tube, the organism is an obligate aërobe; if confined to the closed arm, it is an obligate anaërobe; if, however, the growth is present in both open and closed portions of the tube, it possesses the faculty of growing under either condicion, and is called a facultative organism. 
More exact results can be obtained by the use of special anaërobic culture methods (see p. 82).

Exercise. Note the presence or absence of growth in the closed and open arms of the fermentation tubes inoculated with various cultures (p. 70).
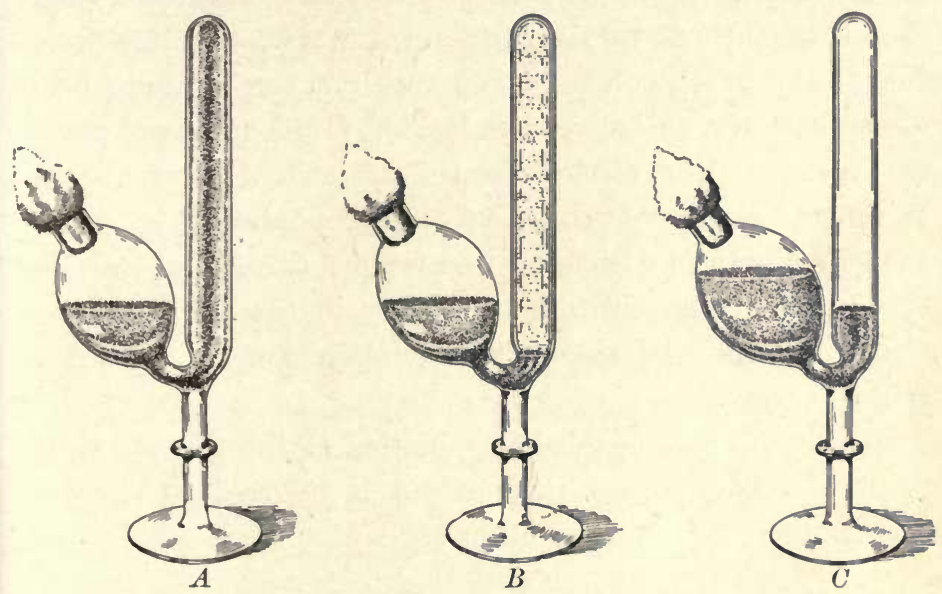

Fig. 14. Types of Growti in Fermentation Tubes $A$, facultative anaërobic organism; $R$, aërobic organism; $C$, gas formation by facultative anaërobic organism. (After Smith)

Relation to temperature. The relation of the developing organism to temperature is determined by noting the comparative rapidity of growth at $20^{\circ} \mathrm{C}$. and at $37^{\circ} \mathrm{C}$. Every organism has a more or less definite temperature range from the minimum to the optimum, and finally to the maximum, temperature at which growth will go on. Generally most bacteria prefer a relatively high temperature, such as blood heat, but some of the fermentative types find ordinary air temperatures almost as suitable as the higher degree of heat.

The ability of the organism to resist the temperature of $80^{\circ} \mathrm{C}$. for fifteen minutes should be determined, since it gives 
indications as to whether the organism produces spores or not. A broth culture forty-eight hours old, grown at $37^{\circ} \mathrm{C}$, is used. In a water bath heated to $80^{\circ} \mathrm{C}$. place four tubes of nutrient broth in immediate contact with the thermometer. After fifteen minutes' exposure to this temperature inoculate each tube, without removing from the water, with three loopfuls of the broth culture, using care not to contaminate the glass above the surface of the liquid. Continue the exposure for fifteen minutes, remove the tubes, and cool at once. Incubate for at least seven days. Organisms producing true endospores are able to withstand this temperature. These are the types that are so difficult to destroy in the sterilization of milk. Organisms resisting this temperature are classed as spore forming.

For the more detailed determination of the thermal death point of the organism the student is referred to standard text-books on general technique.

Exercise. Determine the optimum temperature for growth of the organisms furnished, and also whether or not they produce spores.

Chromogenesis. The formation of pigment is usually most apparent on agar cultures. Cultures should be incubated at both $20^{\circ}$ and $37^{\circ} \mathrm{C}$. Some organisms produce pigment only under special conditions, as $B$. mesentericus ruber when growing on potato. Free oxygen is an essential with practically all types. Therefore the cultures must be under obligate aërobic conditions.

Action on milk. The action of the various organisms on milk is of the very greatest importance to the dairy bacteriologist, and especial attention should be paid to the determination of the points which are enumerated on the following page. 
1. Presence of curd.

2. Time required to curdle.

3. Character of curd, - acid or rennet, homogeneous or showing gas holes, hard or soft.

4. Whey, - amount, clear or turbid, reaction.

5. Digestion of curd, - partial, complete.

6. Digestion without previous curdling.

7. Odor.

8. Gas.

9. Change produced by boiling a few seconds.

10. Amount of acid produced.

Plain milk may be used or that modified with litmus. The reaction of milk or whey may be tested by removing a loopful and placing on delicate litmus paper. Care must be exercised sometimes in discriminating between a rennet and an acid curd. Reliance cannot be placed on the chemical reaction, as a milk curdled with a rennet-like enzyme is generally acid to some extent. The digestion of casein cannot always be determined with certainty in tube cultures. The curd may sometimes shrink in volume and cause extrusion of the whey without undergoing digestive changes. In doubtful cases digestion can be definitely determined by the use of milk agar, which is prepared as follows: A tube of nutrient agar is melted and allowed to cool to $50^{\circ}-55^{\circ} \mathrm{C}$. With a sterile pipette $10-15$ per cent of sterile skim milk is added to it, the mixture being poured into a Petri dish and allowed to solidify. The plate is inoculated with the culture to be tested by making a streak culture with the loop. The action of the organism on the casein is thus localized, and can be noted even though it is very slight. The diffused proteid (casein) is peptonized or rendered soluble by the action of digestive ferments. The action of acid-producing organisms 
when the media contains salt $(\mathrm{NaCl})$ simulates closely that of true digestion, but can be differentiated from it by flooding the plates with dilute $\mathrm{HCl}$ ( 1 per cent), when, in case the change has been due to true digestion, the clear zone about the culture will remain. If the clearing was caused by the solvent effect of the acid, the addition of a larger quantity of acid will cause a reprecipitation.

The presence of gas is easily noted in the case of organisms producing a curd. In other types where no curd is formed, as, for example, in the case of lactose-fermenting yeasts, the presence of gas may be indicated by gently shaking the tube and noting the evolution of bubbles.

Organisms of the same species vary greatly in the amount of acid produced. The amount of acid can be determined in tube cultures by adding to a tube of milk 5 cc. of distilled water. Mix well, turn into a graduated cylinder, note the volume, transfer the mixture to an evaporating dish or an Erlenmeyer flask, add a few drops of the phenolphthalein indicator, and titrate with $\mathrm{N} / 20 \mathrm{NaOH}$. Express the results as per cent of lactic acid.

$$
\text { Per cent of acid }=\frac{\text { cc. of } \mathrm{N} / 20 \times 0.45}{\text { cc. of milk }} .
$$

In studying the various organisms illustrating the different kinds of fermentations four tubes of milk should always be inoculated. Two of these should be incubated at $20^{\circ} \mathrm{C}$. and the remaining two at $37^{\circ} \mathrm{C}$.

Acid fermentation. The normal souring or acid fermentation in milk is usually caused by a number of different kinds of bacteria growing together. In one sample of milk one type may predominate; in a second sample a different type may be most active; in a third still another form may be present in greatest numbers. 
Nearly all of the acid-forming bacteria which are of practical importance may be placed in two great groups: (1) the group including the bacteria which are desirable, especially from the standpoint of the butter and cheese maker, B. lactis acidi Leichmann (Streptococcus lacticus Kruse) being the most important representative of this group; (2) the group including the bacteria which are undesirable in milk, $B$. coli communis and the related form B. lactis aerogenes, both appearing in a number of varieties, being the important representatives. A detailed study should be made of pure cultures of these organisms. In this study attention should be directed to the changes produced in milk, especially to the nature of the eurd formed, the odor and taste of the milk, and the rate and quantity of acid produced at $20^{\circ} \mathrm{C}$. and at $37^{\circ} \mathrm{C}$.

The student should also collect samples of milk, allow them to sour, and judge by the nature of the curd produced the type of organisms concerned in the souring. From the samples of milk showing different types of eurd, plates should be prepared with lactose agar in order to isolate the organisms. Representatives of the various groups should be studied in detail.

Determination of the group to which the organism belongs. The grouping may best be made by the inoculation of litmus-milk tubes from the individual colonies. The results thus obtained should show whether the organism is to be included in either of the groups menitioned above, or belongs to some other.

Exercise. Each student will make a detailed study of the cultures furnished in accordance with the outline given on p. 66. Collect samples of milk, and from them isolate representatives of the two groups of lactic bacteria and study in detail.

Digesting and sweet-curdling fermentation. These changes are produced by various kinds of bacteria. Many of the coccus 
forms present in fresh milk liquefy casein. Spore-bearing bacilli are frequently the cause of these fermentations. The most important representatives of the group are the spore-producing organisms persisting in milk after heating. Various kinds, as shown by their action in litmus milk, should be isolated and studied.

Exercise. Each student will procure samples of milk and heat them to $100^{\circ} \mathrm{C}$. for twenty or thirty minutes. Incubate for six to eight days at $20^{\circ} \mathrm{C}$. From the samples showing curdling and a neutral or alkaline reaction, isolate the causal organisms by means of gelatin plates. Study in detail.

Alcoholic fermentation. The production of alcohol is almost exclusively limited to the action of yeasts. Some bacteria produce alcohol, but in such small quantities that it can be detected only by delicate chemical tests. The ordinary yeasts - beer, wine, and bread yeasts - cannot ferment milk sugar, and hence are of little importance in the dairy. Yeasts that are able to ferment milk sugar are important in the production of fermented drinks from milk, kefir, koumiss, leben, matzoon, etc. Lactose-fermenting yeasts are widely distributed in milk, butter, and cheese, and especially in whey from vats in which a part of the whey is allowed to remain from day to day.

In order to isolate the yeasts from a sample of milk, etc., an acid medium is used. The acidity of the medium may be increased to such a point as to exclude all bacterial growth and still allow yeasts to grow. This is accomplished by adding to the melted lactose agar, just before plating, about 1 per cent of tartaric acid, in the form of a 30 per cent solution. The acid cannot be added to the agar before sterilization as the solidifying properties of the agar would be destroyed. 
In order to determine whether the various types of colonies appearing on the plates are able to ferment lactose, tubes of sterile milk or whey are inoculated, incubated at $20^{\circ} \mathrm{C}$. and at $37^{\circ} \mathrm{C}$. The production of gas $\left(\mathrm{CO}_{2}\right)$, which becomes evident on shaking the tube slightly, is evidence of the fermentation of the lactose. Few of the yeasts produce any curdling of the milk. The presence of alcohol is usually made evident by the odor. The iodoform test for alcohol should be made by distilling over a small quantity from a mass culture in milk or whey, made alkaline with milk of lime. Add to $5 \mathrm{cc}$. of the distillate three or four drops of a 10 per cent solution of iodine in potassium iodide, until a permanent reddish-brown color is obtained. Decolorize by adding 10 per cent $\mathrm{KOH}$ drop by drop. Allow the tube to stand in warm water until a sediment collects. Examine with the $\frac{1}{6}$-inch lens for the characteristic crystals of iodoform. The method should first be tried with distilled water to which a few drops of alcohol have been added.

Ropy fermentation. The peculiar change in milk, known as ropy, slimy, or stringy milk, may be produced by a large number of forms of bacteria. With many it is apparently a normal property, with others it is an evidence of degeneration changes. Some types of lactic-acid bacteria, on cultivation, cease to curdle the milk in a normal manner, but produce in it varying amounts of a slimy material."

The upper layers of cream and milk in bottles may become sliny, due to the growth of aërobic organisms (B. mesentericus vulgatus). The slime-producing organisms proper may or may not produce acid. The organisms studied by Ward, Harrison, etc., belong to the latter group (B. lactis viscosus). The bacteria present in the slimy whey (lange wei) of Holland (Streptococcus Hollandicus), in the taettemoelk of 
Norway (B. lactis longi Troili-Petersson), belong to the acid-producing group.

In the study of this type of fermentation especial attention should be devoted to the cause of the ropy condition. It may be due to the presence of a greatly thickened cell wall (capsule), which may be detected by appropriate staining (p. 48), or to the presence of a mucus-like substance in the milk (galactan).

Exercise. Each student will make a detailed study of the organisms furnished, B. lactis longi.

Bitter fermentation. A bitter taste in milk may be produced by a large variety of bacteria. Many of the digesting bacteria produce a bitter taste in milk, due to the formation of peptones from the albumen and casein. Nonliquefying acid-forming bacteria may produce a specific bitter principle in milk, and the same may be formed by certain types of liquefying acid-producing bacteria.

Exercise. The various milk cultures studied in the digesting and sweet-curdling fermentations should be tested for bitterness.

Cycle of fermentations in milk. Under natural conditions the decomposition of organic matter is accomplished not by a single form of life, but by a greater or less variety of organisms growing together (symbiosis); or one form prepares the way for another, through the establishment of a favorable environment, as in the preparation of a suitable food or a favorable reaction in the medium (metabiosis). Milk is no exception to the rule. During the act of withdrawal from the animal it becomes contaminated with certain classes of bacteria and molds, which make themselves manifest in a constant and regular sequence in ordinary milks. On account of their small numbers it may be impossible to find in 
the fresh milk the various types of bacteria and molds concerned in the changes which the milk will undergo. For instance, it is frequently impossible to demonstrate by plate cultures the presence of acid-forming bacteria or of aciddestroying molds in fresh milk. If, however, the milk is allowed to remain for some days at ordinary temperatures, these forms nearly always appear.

Exercise. In each of two liter flasks should be placed raw skim milk to the depth of 1 inch, the flasks being closed with cotton plugs. One of the flasks should then be heated to $75^{\circ} \mathrm{C}$. for a few moments. Incubate both flasks at $20^{\circ} \mathrm{C}$. for at least a month, noting at frequent intervals the changes occurring.

From the white velvet-like growth appearing on the raw milk (Oidium lactis) microscopic preparations, stained and unstained, should be prepared.

Explain the various changes noted in the two flasks.

Butyric-acid fermentation. In the soil, in manure, and in barn dust are constantly found bacteria that are able to ferment various carbohydrates, such as dextrose and lactose, with the production of butyric acid and other by-products, such as lactic and acetic acids, carbon dioxide, and hydrogen.

Milk usually contains representatives of this group of bacteria. Butter and cheese also contain them, and are sometimes injured by them.

As a group the butyric-acid bacteria are characterized by their relation to oxygen. They are anaërobic, and grow only in the absence of free oxygen when in pure culture. In milk, butter, and cheese the oxygen is consumed by the aërobic forms present; hence the conditions, as far as oxygen is concerned, are favorable for the butyric-acid bacteria. Other conditions are less favorable. The large amount of acid formed in milk and cheese by the lactic-acid bacteria inhibits the butyric-acid bacteria. 
In butter almost no sugar is present. Without it the growth of the butyric-acid bacteria cannot occur.

The butyric-acid bacteria form very resistant spores. Milk that has been heated will frequently undergo an acid fermentation, especially when incubated at $37^{\circ} \mathrm{C}$. Gas is usually abundant. This acid fermentation of heated milk is due to the spores of the butyric-acid bacteria that have not been killed by the heating. Such milk usually has a very offensive odor.

In order to isolate the organisms in pure culture some one of the many ways suggested for the removal of the free oxygen from the culture vessel must be employed. The oxygen can be absorbed by an alkaline solution of pyrogallic acid, or the air may be replaced by an inert gas such as hydrogen. A combination of the two methods is preferable. By exhausting the air from the vessel by means of a water pump, and then filling the vessel with hydrogen and again exhausting, the time required to remove the oxygen may be shortened.

The apparatus illustrated in Fig. 15 will be found very convenient. The Kipp hydrogen generator and two wash bottles, one containing a 10 per cent solution of lead nitrate, the other a solution of silver nitrate, are fastened to a base which is placed on a shelf near a sink. Some form of a Bunsen pump is placed on the wall above the sink: Brass tubing connects the water pump with a mercury manometer and with the gas generator by means of a Y-tube. The third limb of the $Y$-tube is connected to the vessel in which the cultures are to be placed.

In the bottom of a wide-mouthed bottle is placed a layer of sand. A solution of pyrogallic acid is poured upon the sand. The bottle is closed by a rubber stopper provided with one 


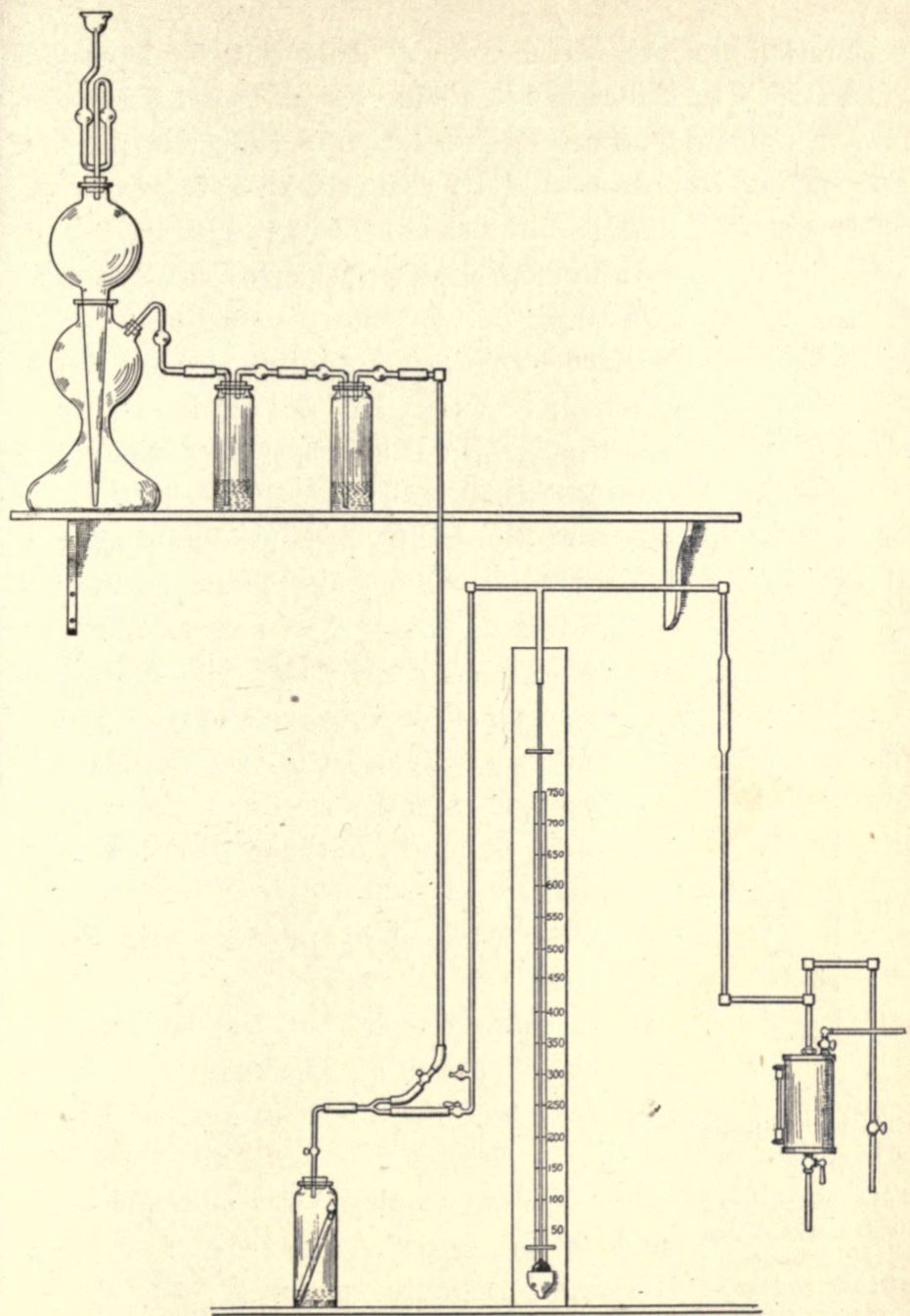

Fig. 15. Apparatus for obtaining Anä̈robic Conditions (After Wesbrook) 
perforation in which is placed a glass tube carrying a groundglass stopcock. The stopcock should be lubricated with vaseline or, preferably, a saturated solution of rubber in paraffin. The rubber stopper should be coated with soft paraffin so

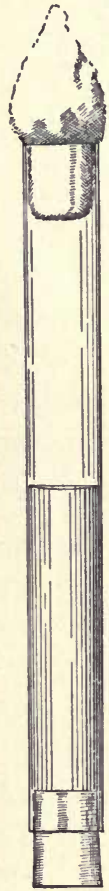

Fig. 16. Burri's

Culture Tube

This tube allows the isolation of anaërobic bacteria without the use of plate cultures that it will not adhere to the glass.

Just before closing the bottle a small amount of a 10 per cent solution of potassium hydrate is added. The cock in the tube leading to the generator is closed, and the air is exhausted from the vessel. The cock between the pump and the $Y$-tube is then closed, while the one admitting the hydrogen is gradually opened. When the jar is filled with hydrogen the exhaustion is repeated. The process must be repeated five or six times. Any slight amount of oxygen remaining will be absorbed by the pyrogallic acid. In order to avoid the forcing of the stopper from the jar, due to the expansion of the hydrogen in the incubator, a negative pressure of $100-150 \mathrm{~mm}$. of mercury should be obtained by partially exhausting the vessel.

Other more simple but less satisfactory methods of obtaining anaërobic conditions will be found described in text-books on general bacteriology.

The medium employed for anaërobic cultures must furnish a source of oxygen. Dextrose is usually employed.

In order to avoid the trouble connected with the use of Petri dishes in anaërobic work, the method devised by Burri is recommended for the isolation of pure 
cultures. Instead of the ordinary culture tube, tubes open at both ends are made from glass tubing. These are stoppered at the lower end with rubber stoppers, but otherwise they are treated as usual. The inoculation of the medium is as usual. Care should be taken to heat the medium for some time in boiling water to expel the dissolved oxygen, to mix very thoroughly with the melted medium the substance to be examined, and to solidify the inoculated medium quickly.

After incubation, those tubes which do not show too numerous colonies, and from which it is desired to make subcultures, have the agar removed by withdrawing the rubber stopper and pushing the agar core into a sterile Petri dish by means of a sterile glass rod. The agar can then be divided with a sterile knife, so as to render any particular colony accessible.

Exercise. Each student will heat to $80^{\circ} \mathrm{C}$. for fifteen minutes a sample of milk inoculated with a small amount of soil. Incubate at $37^{\circ} \mathrm{C}$. If the milk undergoes an acid fermentation, isolate the causal organism by the method described. Make a detailed cultural, morphological, and biochemical study of the organism. 


\section{CHAPTER V}

\section{PRESERVATION OF MILK}

It is impossible to produce milk that does not contain a greater or less number of bacteria, since some of the sources of contamination are of such a nature that they cannot be wholly avoided. A large part of the bacteria that gain entrance to the milk find it a favorable nutrient medium, and products are produced in it as a result of their growth, so that it is rendered more or less unpalatable, or even dangerous as human food.

The period during which milk is fit for use is usually to be measured by hours rather than by days. When the milk is consumed at or near the place of production, the question of preservation is of no great economic importance. With the growth of the modern city, however, the zone from which the milk must be drawn has widened until, in many cases, it is transported for hundreds of miles and does not reach the consumer for twenty-four to forty-eight hours after withdrawal from the animal. It is thus exceedingly desirable that, so far as possible, bacterial life be excluded from milk by the exercise of the most rigid but practicable methods while in the hands of the producer. It is also quite as necessary that the milk while in transit be kept under such conditions as will check bacterial growth.

While the partial exclusion of bacteria will enhance the keeping quality of the product, milk preservation is more particularly limited to those methods by which the organisms 
are more or less completely inhibited or destroyed after they have gained access to the milk.

Various chemical and physical agents are made use of to destroy the bacteria in milk or to prevent their growth. The chemicals used are those known as antiseptic substances, formalin, borax, boracic acid, or proprietary compounds which contain one of these antiseptic substances. Other substances, such as sodium carbonate, by neutralizing the acid produced, lengthen the time before the milk acquires an acid taste. Others, such as hydrogen peroxide, act even more powerfully, destroying the bacteria without altering the taste or appearance of the milk, if they are not used in too large amounts.

The use of practically all chemicals for the preservation of milk is classed as an adulteration and is forbidden by law. The same objection cannot be urged against the use of physical agents, such as heat and cold, employed separately or in combination. The attempt has been made to freeze the milk wholly or in part (Casse system), but this process has not met with much success. Refrigeration or icing, so as to lower the temperature to $4.5^{\circ} \mathrm{C}$. or below, is most successful. While such temperatures prevent in considerable measure the growth of the lactic organisms, there are some species of bacteria capable of multiplying at even very low temperatures $\left(0-1.5^{\circ} \mathrm{C}\right.$.). In refrigerated but unfrozen milk these may develop abundantly, although usually they do not alter the normal characteristics of the milk (taste, smell, etc.) to any great extent.

By the use of high temperatures it is possible to destroy effectively the bacteria present. The temperature at which any form is destroyed is known as its thermal death point. This temperature varies greatly with the organism, depending upon its ability to produce endospores. The exact point is also correlated with the length of time for which the 
exposure is made. Where the vegetative, growing organism is subjected to heat, it is killed at approximately a scalding temperature, varying from $60^{\circ}$ to $80^{\circ} \mathrm{C}$., depending on the period of exposure. Endospores cannot be destroyed unless the temperature is raised to the boiling point or above, and the exposure continued for a considerable time. No process under commercial conditions completely destroys all traces of germ life, so that subsequent chilling of the heated milk is necessary in order to prevent rapid growth of the remaining cells.

In condensed milk the main factor accounting for its keeping qualities is the concentrated nature of the liquid. By the application of heat in a partial vacuum a portion of the water is evaporated. The concentration of the milk is further increased by the addition of cane sugar. The keeping quality is thus dependent upon the same condition that is found in the case of sirups, which "work," i.e. ferment, unless sufficiently concentrated. By heating during the evaporation many of the bacteria are killed. Those remaining are unable to multiply until the milk is diluted with water.

Exercise. EfFect of concentration on bacterial growth. Place about 2 cc. of sweetened condensed milk in each of two sterile test tubes. To one add $10 \mathrm{cc}$. of sterile water. Inoculate both tubes with the same amount of a culture of a digesting organism, and note changes that occur.

Effect of temperature on bacterial growth. The three temperatures which are usually employed for storage of milk under practical conditions are $10^{\circ} \mathrm{C}$. (the temperature of an ordinary ice box), $20^{\circ} \mathrm{C}$. (room temperature), and $30^{\circ}-$ $37^{\circ} \mathrm{C}$. (temperatures approximating blood heat, as when a large can of milk fresh from the cow is allowed to stand without cooling). 
A sample of perfectly fresh milk should be poured into three sterile flasks and incubated at $10^{\circ} \mathrm{C}, 20^{\circ} \mathrm{C}$., and $37^{\circ} \mathrm{C}$. At frequent intervals comparative plate cultures should be made from the samples by the loop method, in order to determine the relative rapidity of growth of bacteria at the various temperatures. The plates should be made at least every twenty-four hours.

Besides this approximate quantitative examination a determination of the rate of acid development should be made by removing 5 cc. of each sample each time the plates are made, and determining the amount of acid therein. The maximum amount of acid formed at the various temperatures should be determined, and the nature and flavor of the curdled milk also noted.

Exercise. Each student will procure a sample of fresh milk (100 cc.), divide the same between three flasks, incubate them at the temperatures indicated, and at intervals of twenty-four hours prepare lactose-agar plates, using the loop method of dilution. Continue until no further increase in acidity is noted.

Pasteurization of milk. A large proportion of the bacteria found in milk are vegetating cells, such forms making up over 99 per cent of the bacterial content of normal milk. The vegetating cells of nearly all forms of bacteria are easily killed at temperatures above $60^{\circ} \mathrm{C}$. In milk thoroughly pasteurized only the spores of spore-forming bacteria are left. To attain this condition it is necessary to heat the milk at such temperatures and for such periods as to alter its chemical and physical nature in some degree. Some of the changes are as follows: The fat globules in normal milk are, to a considerable extent, in clusters or masses, which, on account of having a greater volume for a given surface, are able to overcome the resistance offered by the milk serum, and rise 
rapidly to the surface. If the clusters are broken up and each globule is separated from every other, the creaming is much less rapid. Heating under constant agitation breaks up the aggregates of fat globules and injures the creaming power of the milk. If the individual fat globules are broken into minute fat droplets, as in the case of the so-called "homogeneous milk," the fat cannot be removed, even by passing the milk through a cream separator. The viscosity of cream is diminished when heated on account of the breaking up of the aggregations of fat globules.

Milk contains a substance whose nature is unknown, which has the power of decomposing hydrogen peroxide. If the milk is heated to temperatures above $80^{\circ} \mathrm{C}$., it loses this power. This property is made use of in the Storch test for heated milk.

In commercial practice two methods are used for the pasteurization of milk. They do not differ in principle, but simply in the time and temperature at which the milk is heated. In the intermittent or discontinuous method the milk is heated at temperatures from $60^{\circ}$ to $70^{\circ} \mathrm{C}$. for twenty or thirty minutes, either in bottles or in a container, so arranged that the milk can be heated rapidly and stirred during the process. In the continuous method the milk is heated almost instantaneously to $70^{\circ}-90^{\circ} \mathrm{C}$., maintained there a few seconds, and rapidly cooled. Many types of apparatus have been devised for this purpose, none of which, however, are wholly satisfactory.

Exercise. Examination of milk pasteurized in a discontinUous MACHINE. Each student will procure in a sterile container a sample of raw milk and also a sample of the same milk after it has been pasteurized by the intermittent method at $65^{\circ} \mathrm{C}$., and subsequently cooled. Quantitative lactose-agar plates should be made 
either by the exact dilution method or by the loop method. Determine the percentage of bacteria destroyed by the pasteurizing process.

An examination should also be made, if possible, of milk before and after pasteurization in a continuous machine, and the efficiency of the two methods compared.

Exercise. Effect of heat on the creaming of milk. Heat some whole milk over a water bath to $80^{\circ} \mathrm{C}$. for fifteen minutes, stirring constantly during the heating. Cool at once to at least $20^{\circ} \mathrm{C}$. by placing in cold water; compare the creaming power of the heated sample with that of raw milk by placing a certain amount of each in large test tubes or graduated cylinders and placing in the ice box for twenty-four hours.

In Denimark and in several of the states of the United States it is required by law that all creamery skim milk shall be heated to $80^{\circ} \mathrm{C}$. before it can be returned to the farms. This regulation is for the purpose of preventing the spread of contagious diseases such as tuberculosis and foot and mouth disease, the causal organisms of which are often found in the mixed creamery skim milk. In order to determine whether the creameries are obeying the law, some means of control must be employed, and the Storch test is used for determining whether or not the milk has been heated to the required temperature.

Detection of heated milk. Storch's reaction. The test is made by adding to $5 \mathrm{cc}$. of milk in a test tube one drop of a 0.3 per cent solution of hydrogen peroxide (the commercial product contains 3 per cent of the peroxide), and at once adding 2 drops of a 2 per cent solution of paraphenylendiamin. Mix the reagents thoroughly with the milk. If the milk becomes an intense blue at once, either it has not been heated at all or if heat was applied it was not higher than $78^{\circ} \mathrm{C}$. If the color appears more slowly, after the lapse of half a minute, and becomes a clear grayish blue, the milk has been heated to $79^{\circ}-80^{\circ} \mathrm{C}$. If the milk retains its white 
color unchanged, it has been heated above $80^{\circ} \mathrm{C}$. By the use of this test 10 per cent of milk heated to $78^{\circ} \mathrm{C}$. can be detected when mixed with milk heated above $80^{\circ} \mathrm{C}$.

The test cannot be made with milk in which the acidity has increased to any extent. In the testing of sour milk or buttermilk the sample to be tested must first be neutralized. The blue color does not develop except in the presence of casein. In unheated whey a brown color develops, while in whey heated above $80^{\circ} \mathrm{C}$. no color is produced on adding the reagents.

Exercise. Heat a sample of milk to $70^{\circ} \mathrm{C}$. and cool at once to $20^{\circ} \mathrm{C}$. The heating should be done in such a manner that the sample can be stirred during the process, so as to insure uniform application of heat to all particles of the milk. Apply Storch's test to a portion of the unheated milk and to the heated milk.

Repeat the experiment at $75^{\circ}$ and $80^{\circ} \mathrm{C}$. Note comparative results. 


\section{CHAPTER VI}

\section{RELATION OF BACTERIA TO BUTTER}

Effect of creaming on the distribution of the bacteria in milk. When cream is separated from milk in a centrifugal separator, by virtue of its lighter specific gravity, it is thrown toward the center of the machine. The skim milk and the heavier insoluble particles which constitute the sediment that collects on the wall of the separator bowl are of course thrown to the outside. This differentiation of the milk elements also exerts a material influence on the distribution of bacterial life in the milk. If milk were wholly liquid, i.e. contained none of its normal constituents in other than a soluble condition, the distribution of the bacteria present in the milk would depend entirely upon their specific gravity in comparison to that of milk; but as there are insoluble substances present which are materially lighter (fat), as well as those that are heavier (casein and dirt), than the milk serum, the effect of these materials modify profoundly the distribution of the bacteria. Mud and silt in a body of water carry down the larger part of the microörganistns that may be suspended in the same. So in a similar way the fat globules and the separator slime will be found to act as differential agents in the partial separation of organisms. The immense number of fat globules passing rapidly toward the center of the separator bowl carry with them mechanically a large part of the bacteria in the milk. In gravity creaming the same phenomenon occurs, but to a much less degree. 


\section{Examination of cream and separator slime. The quanti-} tative and qualitative examination of cream may be carried out in the same way as in the case of milk, unless the cream is very thick, in which case the measurement by means of a pipette is too inaccurate. A definite amount of cream (10 grams) may be added to a definite amount of sterile water in a flask by balancing the flask on the scales and adding the desired amount of cream. The cream should then be thoroughly mixed with the water by shaking.

The tenacity of separator slime is such that it can be handled only as a solid. A definite amount is weighed out by placing on the pan of the scales a piece of sterile filter paper $(9 \mathrm{~cm}$. in diameter), and upon this a smaller piece $(7 \mathrm{~cm}$. in diameter). The smaller paper with the slime is placed, by means of sterile forceps, in a definite amount of sterile water and shaken until the slime is completely disintegrated.

Exercise. Each student will make a quantitative examination of (1) skim milk ; (2) cream; (3) separator slime.

The milk should not have an acidity of more than 0.2 per cent. The cream separated should contain at least 30 and preferably 40 per cent of fat. The plates may be prepared by the exact dilution method or by the loop method (p. 28), which will give comparative results.

Examination of sweet and ripened cream. The bacteria in the cream find favorable conditions for growth in case the cream is kept at ordinary temperatures. The growth is confined largely to acid-producing organisms of one form or another. The amount of acid formed will depend on the richness of the cream. The fat is to be looked upon as an inert body so far as acid production is concerned. In determining the acid, the volume taken consists of fat and the liquid part of the cream. In the latter all acid is developed; hence if the fat makes up 30 per cent of the total volume, 
and the acidity is 0.6 per cent, the acidity of the liquid part of the cream amounts to 0.85 per cent. In a 40 per cent cream it will be found impossible to develop much over 0.7 per cent of acid. Such an increase in acid can only occur when the bacteria have greatly increased in number.

Exercise. Each student will make a quantitative and qualitative examination of sweet cream and ripened cream. The sweet cream should be that obtained from very fresh milk. The ripened cream should have developed the acidity necessary for churning. Prepare from each, by the loop method, lactose-litmus-agar plates and also lactose-agar plates. Note which cream shows the greatest diversity of species.

\section{Relation of butter flavor to bacterial development. Two} types of butter are made, one from sweet cream and the other from cream which has been allowed to develop a greater or less amount of acid, - ripened cream. These types of butter differ primarily in flavor. Small samples of cream may be readily churned in Mason fruit jars. The jars should not be filled more than half full, and may be shaken by hand or in a shaking machine. As nearly as possible the process should be the same as that employed on a large scale, washing, salting, etc.

The relation of flavor to bacterial products can be more specifically shown by dividing a quantity of perfectly sweet cream. One portion is to be churned without further treatment; to the other a large amount of starter should be added (25-30 per cent), and the mixture churned. The resulting butters should be examined as to flavor.

Exercise. The samples of cream examined in the previous exercise should be churned and the flavor of the butters noted.

Quantitative analysis of butter. Butter is much more difficult to sample than milk on account of difficulty in securing a thoroughly even emulsion. Two methods are used. 
1. A definite quantity of the butter may be weighed, as for example, 1 gram. If the butter to be sampled is in the form of a pound print, a piece should be cut from one end with a sterile knife, and the sample taken from various portions of the freshly exposed surface. If the butter is in a tub or jar, the sample should be taken with a sterile trier, the plug thus taken split with a knife, and portions taken from various parts of the plug. The butter can be weighed by placing on the pan of the balance a circular piece $(9 \mathrm{~cm}$.) of sterile filter paper. Filter papers of this kind should be sterilized in a Petri dish and kept in stock. On the larger filter place a $7 \mathrm{~cm}$. filter paper, on which the butter is to be placed. The contamination from the air during the weighing process will be of no appreciable importance. The smaller filter with the butter should be transferred by means of sterile forceps to a definite volume of sterile water, which is then heated from $40^{\circ}$ to $45^{\circ} \mathrm{C}$. in order to melt the butter and to distribute the bacteria uniformly. The sample for further dilution should be removed while the contents of the flask are well emulsified.

2. Instead of weighing the butter it may be melted at $40^{\circ}-45^{\circ}$ C. and 1 cc. removed with a warm pipette and transferred to warm sterile water. The pipette should be freed from the adherent fat by filling it with the dilution water a number of times. Further steps in the preparation of the plate cultures and in their study are carried out as described in the case of the examination of milk.

Relation of age of butter to bacterial content. Butter is made up mainly of butter fat, water saturated with salt, and small quantities of the other ingredients of milk. It contains but little proteid matter, and is therefore not well suited for bacterial growth. The difference between the germ content 
of the fresh butter and the same butter some weeks later should be determined.

Exercise. Each student will examine quantitatively a sample of fresh creamery butter. The portion sampled is to be placed in the refrigerator and reëxamined three or four weeks later.

Starters. With increasing knowledge of bacteria and of the various kinds finding favorable conditions for growth in milk and cream, it became evident that the flavor of the butter depended largely upon the types of bacteria present. In order to emphasize the effect of specific forms on the flavor of the product, it is necessary to insure their predominance in the cream by the addition of pure cultures. The three types most commonly present in cream are: (1) lactic-acid-forming organisms of the desirable type (B. lactis acidi Leichmann); (2) acid-producing organisms of less desirable types (B. coli communis, B. lactis aerogenes); (3) organisms liquefying casein (B. fluorescens liquefaciens, etc.).

Pasteurized or perfectly sweet fresh cream should be inoculated with pure cultures of the above types of bacteria, both alone and in mixtures. The growth should be allowed to continue for some time and the flavor of the ripened cream noted. The cream should be churned and the flavor of the butter determined.

These trials may be carried out under more practical conditions by preparing the various starters in the laboratory, and the remaining portion of the work should then be done under creamery conditions.

Commercial starters. With the determination of the importance of the types of bacteria in influencing the flavor of the butter, it became evident that selected forms should be grown in masses and added to the cream to insure their predominance. The introduction of the "pure culture" into 
butter making was largely due to Storch and to Weigmann. Beginning in 1890, the use of pure cultures has spread rapidly and is now quite universally employed in commercial butter production.

On account of the difficulty and trouble the butter maker encounters in selecting milk for the preparation of homemade starters, and the lack of uniformity of product connected with their use, the majority of butter makers prefer to purchase the starter foundation. A number of firms are engaged in the preparation and sale of pure culture or commercial starters for butter making.

These cultures are sold in liquid and dry forms. The liquid starters usually consist of a milk culture of the organism. The dry starters are prepared by mixing with a liquid culture in an actively growing condition some inert substance, as milk sugar, milk powder, etc., in order to absorb the moisture of the culture and thus maintain the organisms in an active condition. In liquid cultures deterioration is rapid on account of the injurious effect of the by-products of the bacteria themselves. In dry cultures deterioration is due mainly to the influence of desiccation on the contained organisms. The effect of desiccation on most lactic bacteria is much less than that of the by-products (acid); hence the starters in dry form can be kept much longer than in liquid form.

The important points to be noted in the examination of commercial starters are: (1) purity; (2) vitality and fermentative power; (3) preservation of original properties on propagation; (4) flavor-producing properties.

Purity. The purity of a starter may be tested by preparing from the original package lactose-agar and plain agar plate cultures. A series of dilutions should be made in order to 
insure plates with a desirable number of colonies, so that all forms present shall have an opportunity to develop.

From liquid starters plates are prepared as in testing the purity of a culture (p. 31). A portion of the dry starter may be transferred to sterile water by dipping the platinum loop. into the water and then into the powder, in which case the moisture will cause a considerable amount of the powder to adhere. From the water blank which has been well shaken after addition of the powder, plates are made in the usual way.

The plates should be carefully studied, the different types of organisms isolated, and a detailed study made of each.

It should be remembered that a dry commercial starter is not necessarily to be condemned because it is not absolutely pure. When propagated the lactic organism will, if properly chosen, develop so much more rapidly than the contaminating organisms that these will not exert any continued effect in the starter or in the cream.

Vitality and fermentative power. In order to be desirable for starter purposes, an organism must grow rapidly when inoculated into milk, which is then kept at temperatures ranging from $20^{\circ}$ to $30^{\circ} \mathrm{C}$; it must also produce acid rapidly, and in sufficient amounts for the production of the desired degree of flavor, and to insure exhaustive chuming.

Since the organisms in the starter as purchased may be few in number and weakened by desiccation or by the accumulation of acid, these points should not be determined by means of cultures inoculated directly from the original package, but the organisms should be brought to a normal state of vitality by inoculating sterile milk from the original starter and incubating for twenty-four hours. From this prepare a second culture, and from this, after twenty-four hours' 
incubation, may be inoculated cultures in order to determine the ability of the organism to produce acid. Into a series of flasks containing a definite amount of sterile milk are inoculated varying amounts of the fresh twenty-four-hour-old milk culture $(1 / 100 \mathrm{cc}$., $1 / 1000 \mathrm{cc}, 1 / 10,000 \mathrm{cc}$. for $100 \mathrm{cc}$. of milk). The flasks should be kept at $25^{\circ}-30^{\circ} \mathrm{C}$. With a culture of high fermentative power 1 part to 1000 of milk should produce curdling in twenty-four hours at $30^{\circ} \mathrm{C}$.

The amount of acid in the curdled milk should be determined by titration with $\mathrm{N} / 20$ sodium hydroxide.

Preservation of propertics on propagation. Many lacticacid cultures when first isolated from milk show desirable properties for use in a starter, but on continued propagation lose them more or less rapidly. They may cease to produce sufficient acid, or they may form it very slowly. Manifestly, such organisms are not well adapted for starter propagation. Their flavor-producing properties may deteriorate, or they may commence to produce a slimy or ropy fermentation in the milk. The ability of an organism to preserve its original properties can only be determined by continued propagation under practical conditions, that is, in the same manner as would be done in a creamery in the carrying on of mother starters in small quantities. The propagation should be carried out under definite conditions as to temperature of incubation and the degree of acidity developed in the starters.

Flavor-producing properties. The ability of the organism to produce a desirable flavor in the starter and in the butter may be determined by frequent examinations of the starters as propagated. The students should be drilled in the judging of starters.

Exercise. Each student will make a detailed study of a commercial starter, as to its purity, vitality, flavor-producing properties, etc. 
Relation of bacteria to keeping quality of butter. In butter from ripened cream the bacterial content is made up almost entirely of lactic-acid bacteria. During the ripening of the cream the other types of bacteria are overgrown by the lactic-acid type, or are even destroyed by the acid produced. In pasteurized-cream butter the percentage of lactic bacteria reaches its maximum, and the pasteurized product has the best keeping qualities. In butter from sweet cream the bacterial content consists of many forms, the lactic bacteria being few in number, and the keeping quality of the butter is impaired.

Exercise. The samples of butter prepared in the exercise on page 95 should be stored in closed vessels in an ordinary refrigerator and examined at intervals of ten days, in order to determine the rate of deterioration of the sweet-cream butter in comparison with that of butter from the ripened cream.

Relation of bacteria in wash water to butter. During the washing of the butter the buttermilk is largely replaced by water with its own peculiar bacterial flora. The germ content of wash waters, qualitatively and quantitatively, varies greatly, depending upon the source, manner of storage, etc. Water from a deep well protected from all surface drainage, or from a protected spring, contains the minimum of bacteria, and will exert but little influence on the quality of the butter.

Water from streams, shallow wells, or ary source unprotected from surface water contains many bacteria and usually of kinds injurious to the butter. On storage in tanks and reservoirs the bacterial content of deep-well and spring waters rapidly increases. This is especially true when the storage tank is not kept perfectly clean. Water freshly pumped is more desirable for use in the dairy than after storage for any length of time. 
It is probable that water which would be pronounced good as the result of a sanitary analysis would be desirable for use in the dairy. For the methods of making a complete bacteriological analysis of water, the student is referred to books on water analysis.

The quality of the water may be determined roughly by the inoculation of dextrose-fermentation tubes with $0.1,1$, and $10 \mathrm{cc}$. of the water. Gas production is indicative of pollution with organic matter, a condition which should not ob-

- tain in a creamery well. The results obtained with the series of fermentation tubes give some idea of the number of gasproducing bacteria present.

Gelatin plates inoculated with varying quantities of the water $(0.1,0.5$, and $1 \mathrm{cc}$.) should show few liquefying bacteria, especially of the fluorescent type (B. fluorescens liquefaciens).

Flasks of sterile milk (100 cc.) should be inoculated with $10 \mathrm{cc}$. of the water, and the nature of the curd and the flavor noted.

Exercise. Each student will test by means of dextrose-fermentation tubes, gelatin plates, and milk, five samples of water representing supposedly good waters and also those polluted with varying amounts of surface drainage. 


\section{CHAPTER VII}

\section{RELATION OF BACTERIA TO CHEESE}

In the making and curing of cheese of all kinds, microorganisms, and especially the bacteria, function as essential factors. Both cheese made with rennet, as well as that produced from curd precipitated by acid, are markedly affected by the activity of these organisms.

Ripening of cheese. Cheese differs from butter in that it must undergo a profound series of changes, physical and chemical in nature, before it is fit for consumption. These changes, collectively known as the curing or the ripening, are exceedingly complex, and differ in details in various types of cheese, but possess in common certain characteristics, the most important of which are (1) the gradual change of the insoluble paracasein to a series of compounds more or less soluble in water, and hence more readily digestible than the original paracasein; (2) the production of substances having more or less distinctive flavors and odors.

Our knowledge relating to the exact nature of these processes is yet far from complete. Moreover, they are of such a complex nature that they cannot be readily demonstrated by laboratory exercises. Consequently only the general principles here involved can be outlined.

The gradual change of the insoluble paracasein to soluble compounds is a process related to digestion, and undoubtedly produced by proteolytic enzymes from various sources. Rennet is added to milk primarily for the purpose of curdling 
it. This effect is due to the presence in the rennet extract of the enzyme rennin, or caseinase, according to modern terminology. Rennet extract also contains pepsin (acid proteinase), which exerts its peculiar action when acid is present, as is normally the case in cheese, due to the action of the lacticacid bacteria on the milk sugar of the whey. An increase in the amount of rennet used in cheese making has been found to hasten the rate at which the water-soluble products are formed, and hence the rapidity of ripening.

In addition to pepsin there is an enzyme normal to milk, known as galactase (alkali proteinase), which also exerts a proteolytic action on casein or paracasein. What rôle the bacteria play in the gradually increasing solubility of the curd is not definitely determined.

Rôle of acid-forming bacteria. In cheddar cheese the initial stages of ripening are closely related to the development of the lactic-acid group of bacteria, as indicated in the following experiment.

Exercise. Melt two tubes of lactose agar, cool to $50{ }^{\circ} \mathrm{C}$, and add to each tube about 15 per cent of sterile skim milk, which is well mixed with the melted agar. If the milk is added to the hot agar, the casein of the milk will be precipitated in flocks. Pour one tube of the mixed agar and milk into a sterile Petri dish and allow it to solidify. Inoculate the remaining tube heavily from a pure culture of a lactic-acid organism and pour into a Petri dish. Keep both plates for twenty-four hours at temperatures favorable for the growth of the lactic-acid organism used. At the end of the period of incubation place on the surface of each plate a strip of filter paper moistened with rennet extract. Incubate the plates at $37^{\circ} \mathrm{C}$. for from two to four hours. At the expiration of this period the following changes are to be noted: On the uninoculated plate the opacity produced by the milk is increased in the immediate neighborhood of the paper, due to a physical change in the casein. On the inoculated plate the casein is changed to soluble compounds, and the opacity is thus destroyed in the immediate neighborhood of the paper. Between the 
clear zone and the unchanged part of the medium will be noted a zone of increased opacity similar to that produced on the uninoculated plate.

Analysis of cheese. As was pointed out in the discussion on general quantitative methods, it is impossible to determine in any sample the absolute number of bacteria present. In the curd and in the cheese the bacteria are held in place exactly as in a gelatin or agar plate. If growth occurs, colonies will result. Thus the distribution of bacteria in cheese is not uniform, but in masses. In the preparation of plate cultures it is essential that as far as possible the masses of bacteria shall be disintegrated. This is done by grinding the cheese with some sharp crystalline substance, as sand, finely ground glass, or sugar. The mixture of cheese and grinding material is suspended in water and well shaken, and plates made therefrom.

Sampling. If an uneut cheese is to be sampled, a plug must be taken with a cheese trier. The rind over the part to be sampled should be removed with a knife, and a plug taken with a sterile trier and transferred to a large test tube or flask. The trier may be sterilized by heating in the flame of a gas burner or of an alcohol lamp, or by dipping in alcohol and igniting it. If a cut cheese is to be sampled, an interior piece should be removed, under aseptic conditions, with a knife and placed in a sterile container. The plug,or piece of cheese is split with a sterile instrument, and pieces removed at various places. A piece of sterile filter paper is placed on the pan of a balance; on this piece of paper is placed a smaller one, also sterile, on which may be placed the cheese for weighing. Exactly 1 gram is weighed out and transferred to a small porcelain mortar, 10 grams of fine sterile quartz sand are added, and the mixture is ground until the cheese 
is disintegrated as completely as possible, when it is transferred to a flask of water by means of a sterile spatula, or rubber-tipped rod.

The flask is well shaken, to free the sand from cheese as far as possible. In transferring with a pipette a portion of this suspension to other water blanks the sample should be taken immediately after shaking, before the sand has settled. Settling may be prevented by holding the pipette in a horizontal position until ready to deliver the contents. The grinding material should be fine enough to avoid clogging the pipette. All the instruments and material with which the cheese comes in contact should be sterile; the contamination from the air during the grinding is so small as to be negligible.

From the various dilutions lactose-agar plates are prepared. The maximum numbers of bacteria are found in cheese from twenty-four to forty-eight hours after making. The number decreases rapidly for some days, reaching at last a level below which farther decrease is very slow. In cheese twenty-four hours old over a billion bacteria per gram may be found.

Exercise. Each student will examine quantitatively samples of cheese from one to two, from ten to fifteen, and from sixty to one hundred days old.

Qualitative examination of milk for cheese making. In milk iytended for cheese manufacture it is desirable that acidforming organisms of the B. lactis acidi type shall be the predominating organisms. As is known, this organism produces no gas in the fermentation of lactose, the curd produced being perfectly homogeneous and pleasant in flavor and odor. Organisms of the B. coli communis and B. lactis aerogenes types are detrimental in cheese, since they cause the cheese to be filled with a greater or less number of holes produced 
by the gas formed from the lactose. The flavor of the cheese is also injured because of other by-products of the acid fermentation.

Tests for the quality of the milk to be used in the manufacture of cheese have for one of their purposes the detection of the second class of organisms, the gas-forming bacteria. If a sample of the milk is placed in a container and incubated at $37^{\circ} \mathrm{C}$. for a few hours, until curdling has taken place, the quality of the milk will be revealed by the nature of the curd. This forms the so-called Gerber fermentation test, largely used in Germany and Switzerland.

A more delicate method is known as the Wisconsin Curd Test, and is carried out as follows: Pint jars are filled with the milk to be tested. The jars should be sterilized before being filled, and care should be taken not to contaminate one sample from others, as by the use of dippers, etc. The jars of milk are warmed to $37^{\circ} \mathrm{C} ., 0.5 \mathrm{cc}$. of rennet extract is added to each jar, and the whole is well mixed. The curd is cut with a case knife. The jars are kept at $37^{\circ}-40^{\circ} \mathrm{C}$., in order to facilitate the shrinking of the curd, as well as the growth of the gas-generating bacteria. After the curd is firm the whey is poured off. Additional portions of whey will be expressed and should also be removed. The jars are kept at the same temperature for eight to twelve hours, and are then examined for flavor, texture, and presence of gas. The particulate bodies in the milk, fat globules and bacteria, are retained in the curd. As the curd shrinks until it occupies but about one sixth of the volume of the milk, the bacteria are concentrated, and the changes produced by them are more apparent. A few gas holes scattered through the total volume of milk, such as is occupied by the curd when produced by the acid-forming bacteria, might pass unnoticed, but, if present in a small mass of 
curd, would be more evident. The curd mass in the Wisconsin Curd Test can also be removed from the jar, its texture determined, the nature of the surface of the curd noted, and thus the presence of undesirable bacteria detected.

This test for the quality of milk is also used in the examination of market milks. It should be remembered that a milk of very high grade may give poor results when tested in this manner, especially if the results are interpreted from the usual standpoint. A curd of fine texture is only possible when the milk contains a considerable number of lactic organisms, since the acid they produce changes the curd so that the small pieces gradually fuse into one mass and show the flaky texture desired by the cheese maker. With a milk produced under such conditions as to exclude the lactic bacteria (certified and sanitary milk), the matting of curd will be largely prevented and the texture will be faulty. On the other hand, a milk of poor quality from the standpoint of the milk consumer, on account of its high bacterial content and consequently poor keeping qualities, may produce a firm, solid curd, free from gas holes.

Each student should make curd tests from various samples of milk. In case milks illustrating the various types cannot be obtained, they may be prepared by inoculating fresh milk with pure cultures of lactic-acid organisms; gas-forming and liquefying bacterial mixtures may also be used. Gas-forming types are readily secured by adding a small quantity of manure to milk. The milk should be inoculated and allowed to stand over night, in order to give a chance for the inoculated organisms to develop before the curd tests are made. If facilities permit, the instructor can have a small cheese (10 pounds or so) made from milk contaminated with gas-forming organisms, so that the course of changes in the cheese 
itself can be followed. In such cases it is advisable to infect the milk on the farm rather than at the factory. This can readily be done by placing in milk cans a small quantity of a gassy starter, so that the normal souring organisms are overpowered.

Exercise. Each student will make curd tests of the samples of milk furnished him. 


\section{CHAPTER VIII}

\section{MILK HYGIENE}

Milk as a distributer of disease. Milk frequently serves as an agent for the transmission of communicable diseases. Such distribution may be from one animal to another of the same or different species; from animal to man, when the disease is one common to both; from man to man, in case of diseases that do not affect the cow, but whose nature is such that milk may be infected with the causal organisms.

The most important of the diseases common to man and cattle is tuberculosis. The milk may contain the tubercle bacilli before being drawn from the udder, as when the mammary gland is one of the organs affected, and even when no disease can be detected in the udder. The milk may be infected after its withdrawal, through the pollution with dust and manure during the milking process.

In tuberculosis of the lungs the tubercular abscesses not infrequently break and discharge their purulent contents into the air passages. This material is coughed up and swallowed by the animal. During the coughing a small part is ejected from the mouth. In the passage through the alimentary tract the tubercle bacilli are uninjured by the digestive juices, the material of which the sputum is composed is digested, and the tubercle bacilli are freed and mixed intimately with the feces. During the milking process more or less manure gains entrance to the milk, and may infect it with the disease-producing organisms. 
But one other disease common to man and cattle is of marked importance because of its direct transmission by means of milk. Foot and mouth disease, the causal organism of which is unknown, is transmitted by milk. The milk is infected before it is drawn.

A considerable number of epidemics of typhoid fever and diphtheria have been traced to infected milk supplies. The organisms causing these diseases always gain entrance to the milk after its withdrawal from the animal. The farm water may become contaminated with typhoid organisms, and the use of such water for washing and rinsing of dairy utensils may serve to contaminate the milk supply.

Convalescents from an attack of the fever may be sources of contamination, if they are concerned in the handling of the milk, or persons serving in the dual capacity of nurse and milkman may be the means of infection. An attack of typhoid fever may be so slight as to pass unnoticed. Such cases known as "walking typhoid" are especially dangerous, since the individual does not recognize the gravity of the situation and accordingly does not use precautionary measures to prevent infection of the milk supply.

Another factor is the "bacillus carrier," a person who may have had typhoid fever years before, but who still harbors in and distributes from his body myriads of typhoid bacteria. Such persons are especially dangerous, as they themselves do not realize the trouble they may cause. The presence of the organism can only be determined by a thorough examination of the dejecta of the individual. It is known that in one case they persisted in the body for over forty-two years.

Diphtheria bacilli in milk may be traced to convalescing patients, or to persons having to do with diphtheria patients and the milk supply. 
It is useless to examine milk for the presence of the organisms of diphtheria or typhoid fever. They do not find favorable conditions for luxuriant growth in milk, in competition with the ordinary milk forms. Again, by the time the milk supply is suspected of being the agent of transmission, the source of contamination has often disappeared and the milk is no longer dangerous. In order to determine the source of an epidemic, a careful historical survey of all the conditions surrounding the outbreak must be made, rather than to seek the causal organism in the water or milk supply by bacteriological methods.

Tubercle bacilli cannot grow in milk after withdrawal from the animal, but they persist for considerable periods in sour milk, butter, and cheese. Milk may be examined for the presence of tubercle bacilli by microscopical methods and by the inoculation of experimental animals.

Microscopical examination for tubercle bacilli. The organism of tuberculosis cannot be stained by the use of ordinary stains. A solution having an intense staining power must be employed. The cells once stained, retain the color even when treated with dilute mineral acids. Thus a preparation may be stained, the color removed from all the material except the tubercle bacilli, and then the preparation restained with a contrast stain.

The microscopical examination of milk for tubercle bacilli is not satisfactory, since these are likely to be present in such small numbers as to be very difficult or impossible to find.

Procedure. The milk should be heated to $70^{\circ} \mathrm{C}$. for a few moments to break up the clusters of fat globules and prevent, as far as possible, the bacteria being carried with the cream in the subsequent process of centrifugalization. Ten to fifteen 
cubic centimeters of the milk are centrifugalized for twenty minutes at a speed of 2000 revolutions per minute. The supernatant liquid is removed by a fine-pointed glass tube attached to an aspirating pump by means of a rubber tube. The cream adhering to the sides of the tube is removed by the use of absorbent cotton. Smears are prepared from the sediment, dried, fixed, and stained as follows: The preparation is flooded with carbol-fuchsin and heated over a water bath or by passing through the flame of a Bunsen burner until the preparation steams. Allow the hot dye to act three to four minutes, and then wash. Decolorize with a 5 per cent solution of nitric acid in 80 per cent alcohol until the red color is discharged. Wash and stain with aqueous methylene blue for a minute. Tubercle bacilli appear as slender red rods on a blue field.

Experience should be gained in the demonstration of tubercle bacilli by the staining and examination of preparations from sputa. The student should bear in mind that the tubercle organism in sputum is longer and more slender than in milk. In sputum it also has a distinct tendency to form a "beaded" appearance.

In manure, and in dust from hay and fodder, there are frequently found organisms; the so-called "acid-fast" bacteria, which possess the same relation to decolorizing agents as the tubercle bacillus. Many of these are very sitmilar in morphology to the tubercle bacillus, differing, however, in cultural characters and in pathogenicity. Through the contamination of the milk with manure and barn dust this type of bacteria is likely to find its way into the milk, and eare must be exercised in the examination of milk sediments not to mistake such forms for true tubercle bacilli. A strict differentiation in such cases can only be made by'animal inoculation. 
Examination for tubercle bacilli by animal inoculation. The inoculation of animals susceptible to tuberculosis is a much more delicate test for the presence of tubercle bacilli in milk than the microscopical examination of the sediment. Highly virulent milk from a tuberculous animal may be diluted as much as a million times, and the presence of tubercle bacilli in the mixed milk may be readily demonstrated by animal inoculation. The guinea pig is used, being the most susceptible of the experimental animals to artificial inoculation, and very resistant to infection in a natural way.

1. The unheated milk may be centrifugalized as for the microscopical examination. Five cubic centimeters of the mixed cream and sediment are injected into the abdominal cavity. The syringe is filled with the mixture, the skin and abdominal wall of the animal picked up between the thumb and finger, and the needle of the syringe thrust through the skin and wall at right angles to the body. The inoculation should be made toward the side rather than in the median line of the abdomen. Frequently the animals die in a short time of acute peritonitis, caused by other organisms present in the milk or on account of a puncture of the intestinal wall in the inoculation process. The animals which survive should be chloroformed in sixty or seventy-five days, and a careful postmortem examination made. The point of inoculation should be examined, as well as the liver, spleen, kidneys, peritoneurn and membranes of the abdominal cavity, and the lymph glands, especially of the inguinal region. The condition of the lungs should also be noted. If lesions are found, the results must be controlled by a microscopical examination of the tissue in smear preparations, and in suspicious cases by a second inoculation of animals with a suspension of the suspected tissues of the first macerated in a mortar with sterile water. The 
suspension may be allowed to settle or filtered through a plug of glass wool, in order to prevent stopping of the needle of the syringe with bits of tissue.

These precautionary measures must be taken, for the injection of large quantities of butter fat into the abdominal cavity of guinea pigs often produces tubercles that are very similar to those produced by the tubercle organism. Inoculation of a second animal with such tissue is without effect.

2. A more rapid method, and one whose delicacy is as great as the intraperitoneal injection of a mixed cream and sediment, is the injection of $1 \mathrm{cc}$. of the whole milk into the muscles of the thigh of a guinea pig. The enlargement of the lymph glands in the inguinal region is evidence of the presence of tubercle bacilli. The changes often appear as early as the twelfth day after inoculation. For more detailed methods of animal inoculation and post-mortem examination, the student is referred to standard works on medical bacteriology.

Exercise. A demonstration of the inoculation and post-mortem examination of an animal should be made by the instructor.

Examination for pyogenic organisms. The pyogenic organism sought for in milk is Streptococcus pyogenes, since this is the most virulent of all the pus-forming bacteria, and the most frequently found in outbreaks of contagious garget. The examination is of little value, except in the case of perfectly fresh market milk, or in high-grade milk whose bacterial content is largely from the interior of the udker. The pyogenic organisms do not find favorable conditions for growth in market milk, and are soon crowded out by lactic organisms; hence in milk of high germ content their detection is difficult.

The streptococci find most favorable conditions for growth on lactose agar at $37^{\circ} \mathrm{C}$. Suspicious colonies on such plates should be inoculated into lactose broth, and this examined 
after twenty-four hours' incubation for chains of cocci, by preparing stained preparations.

The student should become familiar with the morphological, cultural, and physiological characteristics of Streptococcus pyogenes and Staphlococcus pyogenes aureus, the common yellow pus organism.

Microscopical examination of milk. In the milk of all cows is to be found a greater or less number of cellular elements, such as colostrum cells, epithelial cells, and white blood corpuscles. Whenever there is any inflammation in the udder, whether the cause is the presence of pyogenic bacteria or a physiological disturbance such as the influence of cold, bruising, etc., the number of leucocytes is greatly increased. Considerable significance is attached to the presence of large numbers of these cells. The milk drawn from perfectly healthy animals varies so widely in content of leucocytes that it is difficult to establish a standard and to determine what significance they possess. The milk of normal, healthy cows may contain from a few hundred leucocytes to several million per cubic centimeter. In many cities a limit of 500,000 leucocytes per cubic centimeter has been adopted. Milk containing a greater number than this is looked upon as containing pus. While the milk of individual animals in a perfect state of health may frequently exceed this number, it is certain that the mixed milk from a herd of considerable size (twelve animals or more) will not show a higher number unless one or more animals have udder trouble. Standards of this character should, however, be regarded as suggestive rather than as final. Where negative findings are determined they may be taken as indicating a normal, healthy condition; where results show an excess of leucocytes, it should lead to a careful physical examination of the herd. 
Two methods are used for determining the number of leucocytes in milk.

Examination for leucocytes: Doane-Buckley method. Centrifuge tubes, which are graduated at $0.5 \mathrm{cc}$. and $10 \mathrm{cc}$, to aid in filling and in the subsequent removal of the supernatant liquid, are employed. These are filled with the milk to be examined. They are then heated to $70^{\circ}-75^{\circ} \mathrm{C}$. for five to ten minutes, and well shaken after heating. The fat in milk is largely in aggregates of globules, which seem to frequently inclose leucocytes. If the milk is heated, the aggregates of fat globules are broken up and the leucocytes freed. If the unheated milk is used, a large number of leucocytes are to be found in the cream. Since only the sediment can be examined, the leucocytes must be concentrated in it.

The tubes of warm milk are centrifugalized in any of the ordinary types of laboratory centrifuges, or a Babcock tester may be used. Unless a high-speed centrifuge is available, it is difficult to remove the smaller fat globules. If considerable numbers are left, they seriously interfere. with the counting of the leucocytes. These small fat globules may be removed from the milk by the use of high-speed machines, or by centrifugalizing the milk for eight minutes at 2000 revolutions per minute, removing the upper layers as described below, filling with distilled water, and centrifugalizing again for three or four minutes.

The cream and milk are best removed by the use of a fine-pointed glass tube of $2 \mathrm{~mm}$. internal diameter, attached to an aspirating pump by thick-walled rubber tubing. It is advisable to place between the pump and glass tube a bottle to receive the milk. By touching the point of the tube to the liquid the very upper layers may be removed. The fat that adheres to the wall of the centrifuge tube should be wiped 
off with absorbent cotton. The aspirating is continued until but $\frac{1}{2}$ cc. of milk remains. The sediment is well mixed in the liquid by means of a glass rod.

The time of centrifugalization must be sufficient to cause as complete a separation of the fat as possible, since large numbers of fat globules interfere seriously with the counting,

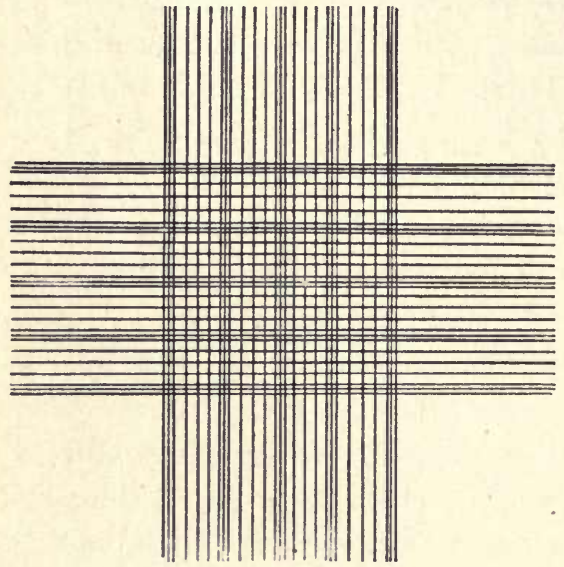

Fig. 17. The Ruled $A$ rea of the ThomaZeiss Bloon Covister

The area of all the squares is $1 \mathrm{sq} . \mathrm{mm}$. The four hundred squares are separated into groups of sixteen by the double lines since they rise to the top of the cell. A speed and time necessary to remove the fat will be sufficient to throw down all leucocytes.

A Thoma-Zeiss blood-corpuscle-counting cell is used. This is formed of a heavy glass slide on which is cemented a square piece of glass having a circular hole in the center. In the center of this hole is cemented on the slide a piece of glass bearing on its upper surface a ruled area made up of 400 squares. The entire ruled area comprises $1 \mathrm{sq}$. mm. The thickness of the glass ring is $0.1 \mathrm{~mm}$. greater than that of the glass bearing the ruled area. Thus, when a perfectly plane piece of glass is used as a cover, the depth of the cell over the ruled area is $0.1 \mathrm{~mm}$; thus the volume of liquid over the rulings is equal to $0.1 \mathrm{cu}$. $\mathrm{mm}$. Each of the 400 squares represents a volume of $1 / 4000 \mathrm{cu} . \mathrm{mm}$. 
With the rod a drop of thoroughly mixed milk and sediment is transferred to the cell and the cover placed in position. The transfer should be made at once after mixing, and the drop should be just large enough to fill the cell completely, or at least three fourths of it, but should not overflow into the moat. The preparation should be allowed to stand a

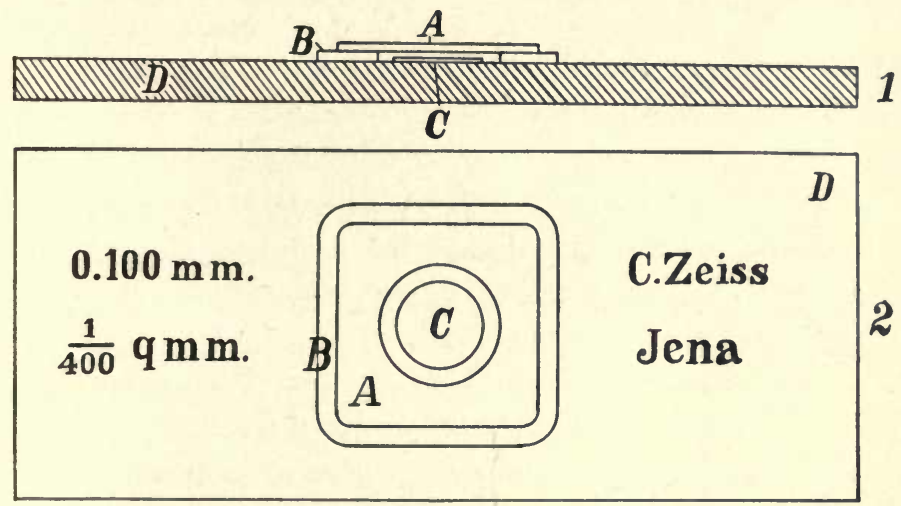

Fig. 18. Thoma-Zeiss Blood-Corpuscle-Counting Cell

$A$, plain glass cover; $B$, glass with circular hole in its center; $C$, glass bearing ruled area. The distance from the upper surface of $C$ to the lower surface of $A$ is $0.1 \mathrm{~mm}$.

moment after filling and covering, to allow the fat globules to rise to the upper portion of the cell and the leucocytes to settle.

The counting is done with a $\frac{1}{6}$-inch objective of long working distance (0.66 numerical aperture), counting the number of leucocytes in the entire ruled area in milks low in content. With increased content a less number of squares may be counted. The small squares will be found divided by double lines into sets of sixteen, in order to facilitate the counting. At least six of these sets should be counted. The average number of leucocytes per small square multiplied by 200,000 
gives the number per cubic centimeter in the original milk. The factor 200,000 is obtained as follows: The leucocytes are concentrated in $1 / 20$ part of the milk. In order to determine the number in each cubic centimeter of the milk, the number found in $1 / 4000 \mathrm{cu}$. $\mathrm{mm}$. of the liquid examined must be multiplied by $\frac{4,000,000}{20}$, or 200,000 .

The student should become familiar with the appearance of white blood corpuscles by the examination of blood. Blood is easily obtained by winding a rubber band about one finger, and when the veins are gorged with blood, pricking oneself at the base of the nail with a glass rod drawn out to a fine point. The puncture should be made by a quick stroke. The drop of blood is mixed with nine volumes of 0.3 per cent glacial acetic acid and examined in the blood counter. The red corpuscles will be scarcely visible in this form of preparation.

Examination for leucocytes; smeared-sediment method. Small glass tubes closed at either end with soft rubber stoppers are used. The tubes should be of $6 \mathrm{~mm}$. internal diameter and of sufficient length to hold $2 \mathrm{cc}$. When filled with milk, which has been heated as in the Doane-Buckley method, they may be centrifugalized in any form of centrifuge. A flat aluminum disk, with upturned edge, may be attached to an ordinary centrifuge. The disk is provided with appropriate clamps for holding the small tubes in position, and with a cover held in place by a screw. With this form of centrifuge head much greater speed can be attained, since the air resistance is much less than with the ordinary tubes. A source of power that would produce $1200-1500$ revolutions per minute with the ordinary head will produce 3000 with the disk. Twenty samples may be centrifugalized at once in an apparatus of this kind. 
After centrifugalization the stopper at the cream end is removed and the layer of cream disturbed with a needle, the milk and cream poured out, and, without placing the tube right side up, the lower stopper with the adhering sediment is removed. The sediment is smeared over a definite area, $4 \mathrm{sq}$. $\mathrm{cm}$. on an ordinary glass slide, by use of the stopper. The smear should be as uniform in distribution as possible. The use of a drop of water will aid in this respect. The smear is allowed to air dry, and is then stained with methylene blue for a few moments. The stain is carefully washed off so as not to loosen the smear, and the slide allowed to dry.

It is examined with an oil-immersion lens, placing the oil directly on the dry smear. The average number of leucocytes per field is determined by counting a number of fields. The number that must be counted depends upon the evenness of distribution of the sediment. At least fifty, should be examined. The diameter of the field of the oil-immersion lens is determined with a stage micrometer. The number of microscopic fields in the total area of the smear is calculated, and from the data thus obtained the average number of leucocytes per cubic centimeter of the milk examined is determined. The student should prepare preparations of blood, staining them with methylene blue, so as to become familiar with the appearance of the white corpuscles when stained. The blood is obtained as previously described. A drop of blood is placed on a clean slide and spread by means of another slide whose corners at one end have been chipped off. This is brought in contact with the drop of blood and drawn along the slide, holding the spreader at an angle of $45^{\circ}$. A thin film of blood is thus deposited along the slide, the broken corners preventing the film extending to the edges of the slide. 
Exercise. Each student will examine samples of milk for leucocytes from healthy animals, and, if possible, from animals having garget. Both methods should be employed on the same sample, if possible. Compare results obtained by the different methods.

Examination for fibrin. In inflammatory troubles of the udder white blood corpuscles are not the only constituent of the blood passing from the blood vessels into the glandular tissue and milk ducts. The blood serum, with its dissolved constituents, serum albumen and fibrinogen, passes out to a greater or less extent. Under appropriate conditions the fibrinogen is changed to the insoluble fibrin, which, when present in any considerable amounts, can be detected by microscopic examination. The fibrin determines the distribution of the leucocytes. When fibrin is present in small quantities or not at all, the leucocytes are evenly distributed. In the presence of fibrin larger or smaller clumps are to be found.

The milk may be centrifugalized, as in the smeared-sediment method for leucocytes. The sediment should be spread on a slide, noting whether any dough-like material is present. The smear is stained as follows, after being allowed to thoroughly air dry : Flood with aniline-water gentian violet (p. 43). Allow stain to remain on the preparation for five minutes, and then wash. Flood with iodine solution (p. 43), wash off after one or two minutes, and add aniline oil from time to time until the color is completely discharged, as shown by the colorless condition of the oil. Wash in water, dry, and examine. The fibrin should appear as threads stained dark purple.

Exercise. The student should familiarize himself with the appearance of fibrin in blood. Examine normal milk for fibrin; also that from an animal having garget.

Direct enumeration of bacteria in milk. In control work, such as must be carried on in the municipal laboratories of 
cities which have established a numerical standard in reference to the bacterial content of milk, it is desirable to have some method by which it can quickly and easily be determined whether or not a sample of milk exceeds the established standard. The analyst does not care to know the exact number of organisms present, but merely whether the milk is good, fair, or poor, with reference to the standard adopted. The smear prepared from the milk for determination of the number of leucocytes present will serve for this purpose. The average number of bacteria per field of the oil-immersion lens is determined by counting those appearing in a number of fields. From the average per field and the areas of field and smear the number of bacteria per cubic centimeter of milk may be calculated. Each diplococcus or diplobacillus, each chain or clump of organisms, should be counted as one, since such aggregations will usually produce but one colony on the plate cultures. The legal standard always refers to the number of organisms as determined by some method of plating.

The value of this method depends much on the experience of the analyst. With considerable experience it is certain that in many cases it is possible to determine whether a given sample falls below or greatly exceeds the standard. Standards of this character, however, should not be interpreted too strictly. They serve as admirable aids in milk control, inasmuch as the inspector is thus able to concentrate his attention on those cases which are désignated as suspicious by the laboratory analysis. Where direct tests show a perfectly normal condition, plating is unnecessary, and only the questionable samples need be further examined. The exact counting is not necessary, a rapid glance over the preparation enabling the experienced analyst to judge of the quality of the milk. The examination also gives the analyst 
some idea as to the dilutions to be used in the preparation of plate cultures, so as to obtain plates with the desired number of colonies.

Microscopic examination for streptococci. As has been previously pointed out, the pyogenic organisms most likely to be the cause of udder troubles are streptococci of the type of Streptococcus pyogenes. In the examination of the smears prepared from the milk sediments, the form of organisms predominating should be noted. The value of the examination depends much upon the age of the milk. It is impossible to distinguish certain types of lactic-acid organisms from pyogenic streptococci, since their morphology is so nearly identical. Thus, in milk, in which the lactic organisms have increased in numbers, such an examination is of doubtful value. In perfectly fresh milk long chains of streptococci are indicative of udder trouble. In milk of animals suffering from garget immense numbers of such organisms may frequently be found in the sediment. 


\section{APPENDIX A}

\section{PIPETTES}

Preparation of pipettes. The quantitative bacteriological analyses of milk and other dairy products involves the use of a large amount of glassware, especially pipettes. One-cc. pipettes, suitable for use in quantitative work, can be easily prepared. Glass tubing of $4 \mathrm{~mm}$. internal diameter is cut into pieces 20 inches long. The piece is heated in the center and drawn out so as to form the tips of the pipettes. The pieces are then cut in two, and both ends slightly fused in the flame to remove the sharp edges. The pipettes are rendered perfectly free from grease and dirt by boiling them in a solution of washing powder, rinsing well with distilled water, and heating in a hot-air sterilizer for an hour at $175^{\circ}-200^{\circ} \mathrm{C}$., or by washing in alcohol and then in ether.

A Schuster dropping bottle is thoroughly cleaned and dried. The top of the bottle should be drawn out so it can be easily introduced within the lumen of the pipette, and the hole in the bottle made very small. In the

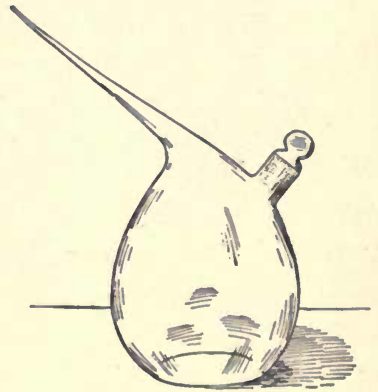

Fig. 19. Schuster Dropping BotTLE

The top of the bottle is drawn so as to form a capillary tube from which the mercury will run slowly bottle are placed 13.6 grams of perfectly clean, dry mercury. The upper part of each pipette is coated with a thin coat of paraffin by warming the glass and rubbing it on a piece of paraffin. The pipette is held between the first two fingers, the tip resting on the ball of the thumb. The mercury is poured into the pipette slowly so as to allow the air to escape. $\Lambda$ very small bubble of air will usually be retained in the tip, but this may be neglected. The upper level of the mercury which indicates a volume of one cc. is marked on the paraffin by a pin scratch. The mercury is returned to the dropping 
bottle by allowing it to run from the top of the pipette, and the operation repeated with each pipette. If the glassware is free from moisture and grease, and the mercury clean, there should be no adherence of the mercury to the glass and no tendency for it to form small globules. A permanent mark is placed on the pipettes by scratching through the paraffin with a fine needle and filling the scratch with hydrofluoric acid or diamond ink and allowing the same to act for five minutes; then wash off, remove paraffin by warming sufficiently to melt it, and wipe with paper. A mark may be made by the use of a sharp file, which method avoids the use of paraffin and hydrofluoric acid. 


\section{APPENDIX B}

\section{DESCRIPTIVE CHART, SOCIETY OF AMERICAN BACTERIOLOGISTS}

Prepared by F. D. Chester, F. P. Gorham, Erwin F. Smith, Committee on Methods of Identification of Bacterial Species

\section{DETAILED FEATURES}

Note. Underscore required terms.

\section{MORPHOLOGY}

1. Vegetative cells, medium used temp. , age days

Form, round, short rods, long rods, short chains, long chains, filaments, commas, short spirals, long spirals, clostridium, cuneate, clavate, curved.

Limits of size

Size of majority.

Ends, rounded, truncate, concave.
Agar hanging
block
Orientation (grouping)
Chains (number of elements)
Short chains, long chains.
Orientation of chains, parallel, irregular.

2. Sporangia, medium used , temp. age.

$$
\text { days }
$$

Form, elliptical, short rods, spindled, clavate, drumsticks.

Limits of size. Size of majority Agar hanging
block

Orientation (grouping) Chains (number of elements) Orientation of chains, parallel, irregular.

Location of endospores, central, polar.

\section{Endospores.}

Form, round, elliptical, elongated.

Limits of size.

Size of majority 
Wall, thick, thin.

Sporangium wall, adherent, not adherent.

Germination, equatorial, oblique, polar, bipolar, by stretching.

4. Flagella No. Attachment, polar, bipolar, peritrichiate.

How stained

5. Capsules, present on

6. Zoöglœa, Pseudozoöglœa.

7. Involution forms, on. in. days at $\circ \mathrm{C}$.

8. Staining reactions.

$1: 10$ watery fuchsin, gentian vio'et, carbol-fuchsin, Loffler's alkaline methylene blue.

Special stains.

Gram. Glycogen

Fat Acid fast

Neisser

\section{CULTURAL FEATURES}

\section{Agar stroke.}

Growth, invisille, scanty, molerate, abundant.

Form of growth, filiform, echinulate, beaded, spreading, plumose, arborescent, rhizoid.

Elevation of growth, flat, effuse, raised, convex.

Luster, glistening, dull, cretaceous.

Topography, smooth, contourer, rugose, verrucose.

Optical characters, opaque, translucent, opalescent, iridescent.

Chromogenesis (5) .

Odor, absent, decided, resenbling

Consistency, slimy, butyrous, viscid, membranous, coriaceous, brittle.

Medium, grayed, browned, reddened, blued, greened.

\section{Potato.}

Growth, scanty, moderate, abundant, transient, persistent.

Form of growth, filiform, echinulate, beaded, sprealing, plumose, arborescent, rhizoid.

Elevation of growth, flat, effuse, raised, convex.

Luster, glistening, dull, cretaceous.

Topography, smooth, contoured, rugose, verrucose.

Chromogenesis (5) Pigment in water, insoluble, soluble; other solvents

Odor, absent, decided, resembling. 
Consistency, slimy, butyrous, viscid, membranous, coriaceous, brittle. Medium, grayed, browned, reddened, blued, greened.

\section{Lœffler's blood serum.}

Stroke, invisible, scanty, moderate, abundant.

Form of growth, filiform, echinulate, beaded, spreading, plumose, arborescent, rhizoid.

Elevation of growth, flat, effuse, raised, convex.

Luster, glistening, dull, cretaceous.

Topography, smooth, contoured, rugose, verrucose.

Chromogenesis (5)

Medium, grayed, browned, redclened, blued, greened.

Liquefaction begins in....................d., complete in

4. Agar stab.

Growth, uniform, best at top; best at bottom; surface growth scanty, abundant; restricted, widespread.

Line of puncture, filiform, beaded, papillate, villous, plumose, arborescent; liquefaction.

5. Gelatin stab.

Growth, uniform, best at top, best at bottom.

Line of puncture, filiform, beaded, papillate, villous, plumose, arborescent.

Liquefaction, crateriform, napiform, infundibuliform, saccate, stratiform; begins in. d., complete in

Medium, fluorescent, browned

\section{Nutrient broth.}

Surface growth, ring, pellicle, flocculent, membranous, none.

Clouding, slight, moderate, strong; transient, persistent; none; fluid turbid.

Odor, absent, decided, resembling

Sediment, compact, flocculent, granular, flaky, viscid on agitation, abundant, scant.

\section{Milk.}

Clearing without coagulation.

Coagulation, prompt, delayed, absent.

Extrusion of whey, begins in

Coagulum, slowly peptonized, rapidly peptonized.

Peptonization begins on......................, complete on

Reaction, $1 \mathrm{~d}$. $2 \mathrm{~d}$. $4 \mathrm{~d}$. $\therefore, 10 \mathrm{~d}$. $20 \mathrm{~d}$.

Consistency, slimy, viseid, unchanged.

Medium, browned, reddened, blued, greened.

Lab ferment, present, absent. 


\section{Litmus milk.}

Acid, alkaline, acid then alkaline, no change.

Prompt reduction, no reduction, partial slow reduction.

9. Gelatin colonies.

Growth, slow, rapid.

Form, punctiform, round, irregular, amoboid, mycelioid, flamentous, rhizoid.

Elevation, flat, effuse, raised, convex, pulvinate, crateriform, (liquefying).

Edge, entire, undulate, lobate, erose, lacerate, fimbriate, filamentous, floccose, curled.

Liquefaction, cup, saucer, spreading.

\section{Agar colonies.}

Growth, slow, rapid (temperature

Form, punctiform, round, irregular, amoboid, mycelioid, filamentous, rhizoid.

Surface, sinooth, rough, concentrically ringed, radiate, striate.

Elevation, flat, effuse, raised, convex, pulvinate, umbonate.

Edge, entire, undulate, lobate, erose, lacerate, fimbriate, floccose, curled.

Internal structure, amorphous, finely granular, coarsely granular, grumose, filamentous, floccose, curled.

\section{Starch jelly.}

Growth, scanty, copious.

Diastasic action, absent, feeble, profound.

Medium stained

12. Silicate jelly (Fermi's solution).

Growth, copious, scanty, absent.

Medium stained

\section{Cohn's solution.}

Growth, copious, scanty, absent.

Medium, fluorescent, nonfluorescent.

\section{Uschinsky's solution.}

Growth, copious, scanty, absent.

Fluid, viscid, not viscid.

\section{Sodium chloride in bouillon.}

Per cent inhibiting growth.

16. Growth in bouillon over chloroform, unrestrained, feelle, absent.

17. Nitrogen.

Obtained from peptone, asparagin, glycocoll, urea, ammonia salts, nitrogen. 
18. Best media for long-continued growth.

\section{Quick tests for differential purposes}

\section{PHYSICAL AND BIOCHEMICAL FEATURES}

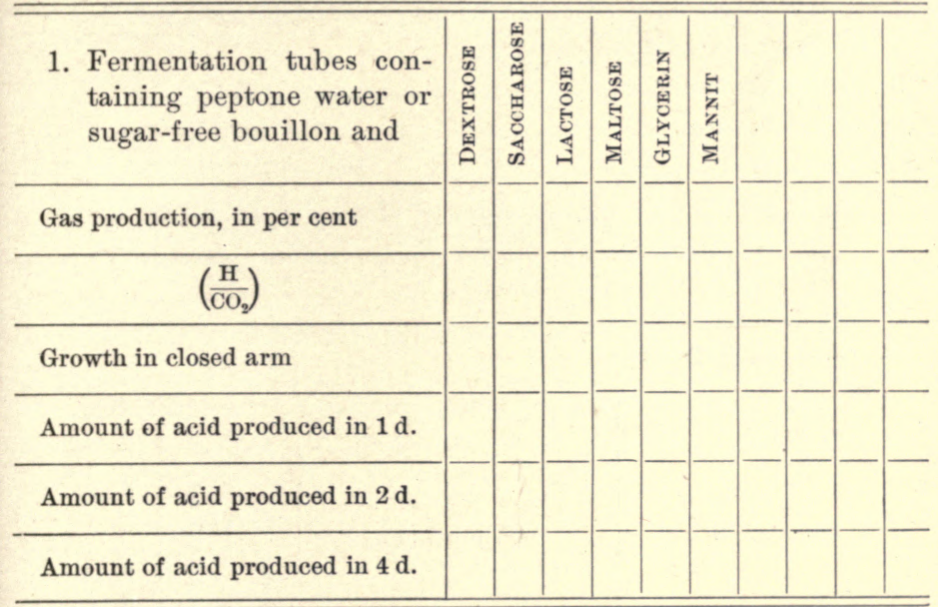

2. Ammonia production, feeble, moderate, strong, absent, masked by acids.

3. Nitrates in nitrate broth, reduced, not reduced.

Presence of nitrites ammonia.

" " nitrates. free nitrogen

4. Indol production, feeble, moderate, strong.

5. Toleration of acids, great, medium, slight.

Acids tested.

6. Toleration of $\mathrm{NaOH}$, great, medium, slight.

7. Optimum reaction for growth in bouillon, stated in terms of Fuller's scale.

8. Vitality on culture media, brief, moderate, long.

9. Temperature relations :

Thermal death point (10 minutes' exposure in nutrient broth when this is adapted to growth of organism).

Optimum temperature for growth.................., or best growth at $15^{\circ} \mathrm{C} ., 20^{\circ} \mathrm{C} ., 25^{\circ} \mathrm{C} ., 30^{\circ} \mathrm{C} ., 37^{\circ} \mathrm{C} ., 40^{\circ} \mathrm{C} ., 50^{\circ} \mathrm{C} ., 60^{\circ} \mathrm{C}$. 
Maximum temperature for growth

Minimum temperature for growth

10. Killed readily by drying : resistant to drying.

11. Per cent killed by freezing (salt and crushed ice or liquid air)

12. Sunlight : exposure on ice in thinly sown agar plates : one half plate covered (time 15 minutes), sensitive, not sensitive. Per cent killed

13. Acids produced

14. Alkalies produced

15. Alcohols

16. Ferments : pepsin, trypsin, diastase, invertase, pectase, cytase, tyrosinase, oxidase, peroxidase, lipase, catalase, glucase, galactase, $l a b$, etc.

17. Crystals formed

18. Effect of germicides.

\begin{tabular}{|c|c|c|c|c|c|}
\hline Substance & Method Used & $\begin{array}{l}\text { 㽞 } \\
\text { 总 } \\
\text { 至 }\end{array}$ & 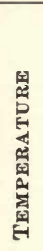 & 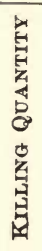 & 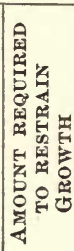 \\
\hline & & & & & \\
\hline & & & & & \\
\hline & & & & & \\
\hline & & - & & & \\
\hline & & & & & \\
\hline & & & & & \\
\hline & & & & & \\
\hline & & & & & \\
\hline & & & & & \\
\hline
\end{tabular}




\section{PATHOGENICITY}

1. Pathogenic to animals.

Insects, crustaceans, fishes, reptiles, birds, mice, rats, guinea pigs, rabbits, dogs, cats, sheep, goats, cattle, horses, monkeys, man.

\section{Pathogenic to plants.}

3. Toxins, soluble, endotoxins.

4. Nontoxin forming.

5. Immunity bactericidal.

6. Immunity nonbactericidal.

7. Loss of virulence on culture media : prompt, gradual, not observed in. months.

\section{BRIEF CHARACTERIZATION}

Mark + or $\mathrm{O}$, and when two terms occur on a line erase the one which does not apply unless both apply

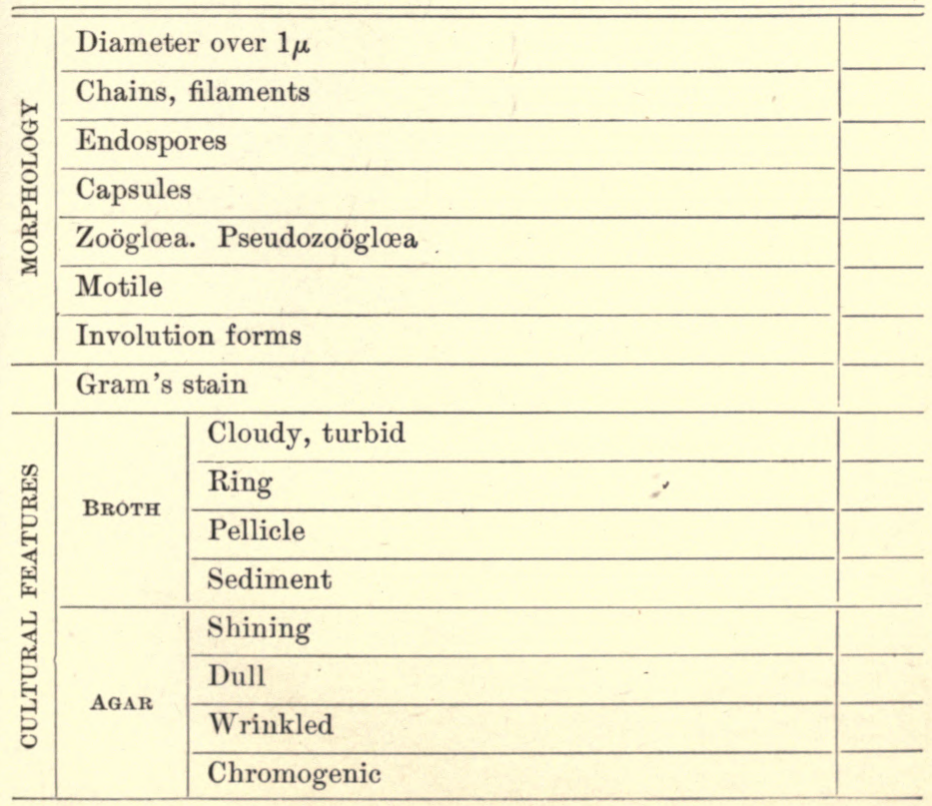




\section{BRIEF CHARACTERIZATION (continued)}

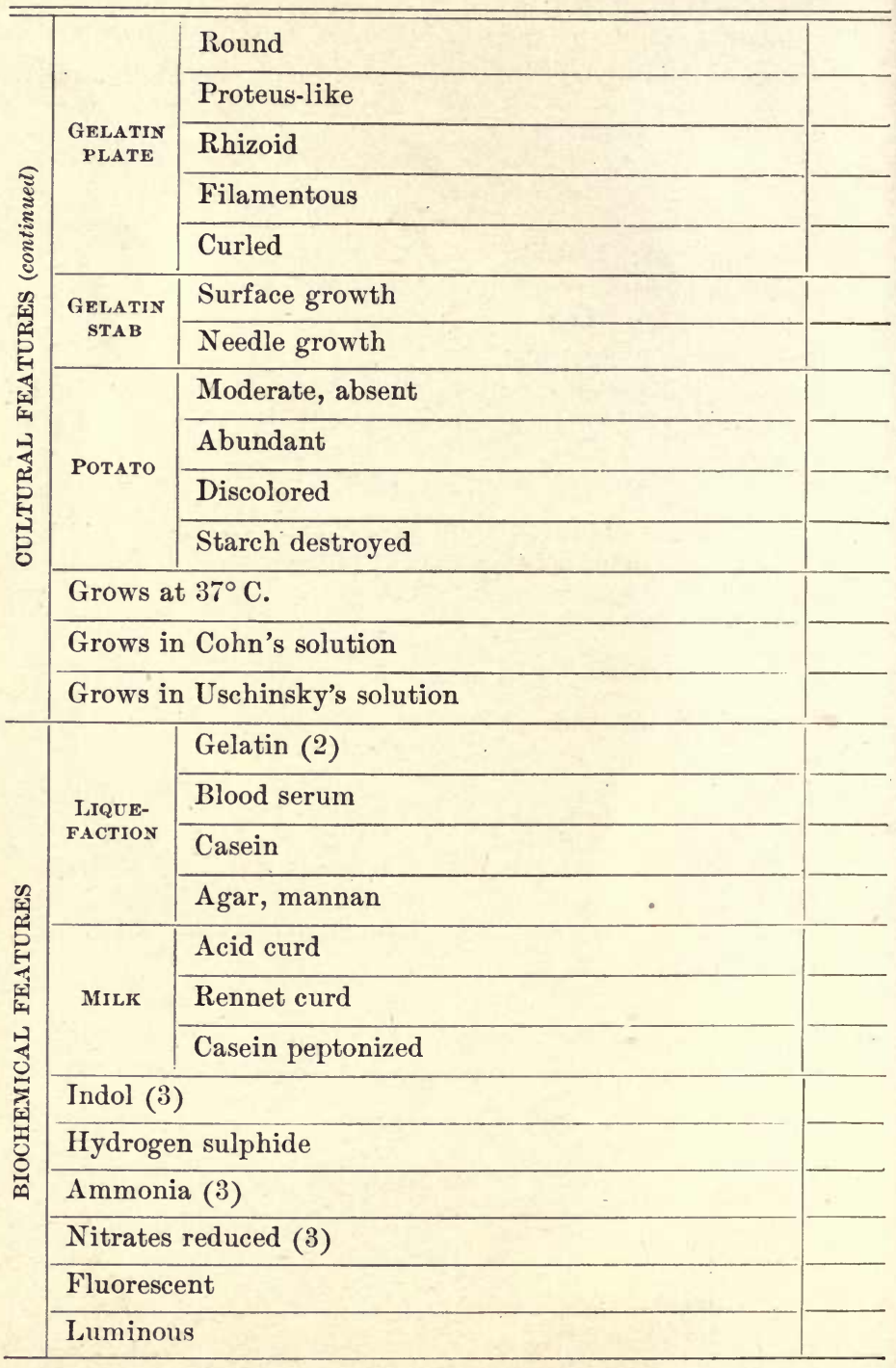




\section{BRIEF CHARACTERIZATION (continued)}

\begin{tabular}{l|l|l}
\hline \hline & Animal pathogen, epizoön & \\
\hline & Plant pathogen, epiphyte & \\
\hline Soil & Milk & \\
\hline \multirow{2}{*}{} & Fresh water & \\
\hline Salt water & Sewage & \\
\hline Iron bacterium & \\
\hline Sulphur bacterium & \\
\hline
\end{tabular}

\section{NOTES}

(1) For decimal system of group numbers see Table I. This will be found useful as a quick method of showing close relationships inside the genus, but is not a sufficient characterization of any organism.

(2) Gelatin stab cultures shall be held for six weeks to determine liquefaction.

(3) Ammonia and indol tests shall be made at the end of the tenth day, nitrite tests at the end of the fifth day.

(4) Generic nomenclature shall begin with the year 1872 (Cohn's first important paper).

Species nomenclature shall begin with the year 1880 (Koch's discovery of the poured-plate method for the separation of organisms).

(5) Chromogenesis shall be recorded in standard color terms. 


\section{TABLE I}

A NUMERICAL SYSTEM OF RECORDING THE SALIENT CHARACTERS OF AN ORGANISM (GROUP NUMBER)

100.

200.

10.

20.

30 .

1.

2.

0.1

0.2

0.3

0.4

.01

.02

.03

.04

.001

.002

.003

.004

.0001

.0002

.0003

.00001

.00002

.00003

.00004

.00005

.00006

.00007

.00008

.00009

.00000

.000001

.000002

.000003
Endospores produced

Endospores not produced

Aërobic (strict)

Facultative anaërobic

Anaërobic (strict)

Gelatin liquefied

Gelatin not liquefied

Acid and gas from dextrose

Acid without gas from dextrose

No acid from dextrose

No growth with dextrose

Acid and gas from lactose

Acid without gas from lactose

No acid from lactose

No growth with lactose

Acid and gas from saccharose

Acid without gas from saccharose

No acid from saccharose

No growth with saccharose

Nitrates reduced with evolution of gas

Nitrates not reduced

Nitrates reduced without gas formation

Fluorescent

'Violet chromogens

Blue chromogens

Green chromogens

Yellow chromogens

Orange chromogens

Red chromogens

Brown chromogens

Pink chromogens

Nonchromogenic

Diastasic action on potato starch (strong)

Diastasic action on potato starch (feeble)

Diastasic action on potato starch (absent) 
.0000001 Acid and gas from glỳcerin

.0000002 Acid without gas from glycerin

.0000003 No acid from glycerin

.0000004 No growth with glycerin

The genus according to the system of Migula is given its proper symbol, which precedes the number thus:

Bacillus coli (Esch.) Mig. Bacillus alcaligenes Petr. Pseudomonas campestris (Pam.) Sm. Bacterium suicida Mig. becomes B. 222.111102

“ B. 212.333102

“ Ps. 211.333151

“ $\quad$ Bact. 222.232?03 


\section{APPENDIX C}

\section{CONVERSION FACTORS FOR THERMOMETER SCALES}

To change Centigrade to Fahrenheit: $($ C. $\times 9 / 5)+32=F$.

To change Fahrenheit to Centigrade: $(\mathrm{F} .-32) \times 5 / 9=\mathrm{C}$.

To change Reaumur to Fahrenheit: $($ R. $\times 9 / 4)+32=\mathrm{F}$.

To change Fahrenheit to Reaumur: $($ F. -32$) \times 4 / 9=\mathbf{R}$.

To change Centigrade to Reaumur: C. $\times 4 / 5=R$.

To change Reaumur to Centigrade: R. $\times 5 / 4=$ C.

\section{CONVERSION FACTORS}

METRIC TO ENGLISH, ENGLISH TO METRIC

Meter $=39.3704$ inches

Millimeter $=0.03937$ inches (0.04 approximately)

Inch $=25.3997 \mathrm{~mm}$. (25.4 approximately)

Liter $=2.11$ pints $(1$ quart approximately)

Cubic centimeter $=16.23$ minims

Fluid ounce $=29.578$ cubic centimeters ( 30 cc. approximately)

Gram $=15.432$ grains

Kilogram $=2.204$ avoirdupois pounds

Ounce avoirdupois $=28.349$ grams

Pound avoirdupois $=453.584$ grams

Ounce troy $=31.103$ grams

Square centimeter $=0.1548$ square inches

Square inch $=6$ square centimeters (approximately)

Cubic centimeter $=0.0609$ cubic inches

Cubic inch $=16$ cubic centimeters (approximately) 


\section{APPENDIX D}

\section{APPARATUS FOR EACH STUDENT}

1 Erlenmeyer flask, 1000 cc.

2 Erlenmeyer flasks, $600 \mathrm{cc}$.

2 Erlenmeyer flasks, $300 \mathrm{cc}$.

10 Erlenmeyer flasks, $150 \mathrm{cc}$.

200 test tubes $(15 \times 120 \mathrm{~mm}$. $)$

30 Petri dishes $(90 \mathrm{~mm}$. diameter)

10 glass tumblers

5 1-cc. pipettes, graduated in tenths of a cc.

5 1-cc. pipettes (volume)

15 -cc. pipette (volume)

1 pipette case

8 stain bottles in tray

3 wire baskets $(4 \times 4 \times 4$ inches $)$

1 Bunsen burner and tubing

1 thermometer $\left(0^{\circ}-100^{\circ} \mathrm{C}\right.$.)

2 glass rods for platinum needles

2 stirring rods

1 test-tube cleaner

1 rice cooker

1 ring stand, 2 rings

1 burette clamp

1 tripod

24 -in. funnels

13 -in. casserole

1 100-cc. graduated cylinder

1 copper cup

$10 \mathrm{~cm}$. rubber tubing with glass tip

1 pincheock

1 piece wire gauze $12 \mathrm{~cm}$. square

1 piece Russia iron $12 \mathrm{~cm}$. square

$\frac{1}{2}$ ounce cover glasses $18 \mathrm{~mm}$. square, $0.17 \mathrm{~mm}$. thick (No. 2)

50 glass slides $25 \times 75 \mathrm{~mm}$.

1 pair cover-glass forceps

1 pair fine-pointed forceps

1 hollow-ground slide

$140 \mathrm{~mm}$. No. 27 platinum wire 


\section{GLOSSARY OF TERMS}

amœboid, assuming various shapes like an amøba.

amorphous, without visible differentiation in structure.

arborescent, a branched, tree-like growth.

beaded, in stab or stroke, disjointed or semiconfluent colonies along the line of inoculation.

brittle, growth dry, friable under the platinum needle.

bullate, growth rising in convex prominences, like a blistered surface.

butyrous, growth of a butter-like consistency.

chains, short chains, composed of 2-8 elements; long chains, composed of more than 8 elements. ciliate, having fine, hair-like extensions, like cilia.

cloudy, said of fluid cultures which do not contain pseudozoöglœeæ.

coagulation, the separation of casein from whey in milk. This may take place quickly or slowly, and as the result either of the formation of an acid or of a lab ferment.

contoured, an irregular, smoothly undulating surface, like that of a relief map. convex, surface the segment of a circle, but flattened.

coprophyl, dung bacteria. coriaceous, growth tough, leathery, not yielding to the platinum needle.

crateriform, round, depressed, due to the liquefaction of the medium.

cretaceous, growth opaque and white, chalky.

curled, composed of parallel chains in wavy strands, as in anthrax colonies.

diastasicaction (sameas diastatic), conversion of starch into watersoluble substances by diastase. echinulate, in agar stroke a growth along line of inoculation, with toothed or pointed margins ; in stab cultures, growth beset with pointed outgrowths.

effuse, growth thin, veily, unusually spreading.

entire, smooth, having a margin destitute of teeth or notches. erose, border irregularly toothed. filamentous, growth composed of long, irregularly placed or interwoven filaments.

filiform, in stroke or stab cultures a uniform growth along line of inoculation. 
fimbriate, border fringed with slender processes, larger than filaments.

floccose, growth composed of short curved chains, variously oriented.

flocculent, said of fluids which contain pseudozoöglœe, i.e. small adherent masses of bacteria of various shapes and floating in the culture fluid. fluorescent, having one color by transmitted light and another by reflected light.

grumose, clotted.

infundibuliform, form of a funnel or inverted cone.

iridescent, like mother-of-pearl. The effect of very thin films. lacerate, having the margin cut into irregular segments as if torn.

lobate, border deeply undulate, producing lobes (see undulate).

maximum temperature, temperature above which growth does not take place.

membranous, growth thin, coherent, like a membrane.

minimum temperature, temperature below which growth does not take place.

mycelioid, colonies having the radiately filamentous appearance of mold colonies.

napiform, liquefaction with the form of a turnip.

nitrogen requirements, the necessary nitrogenous food. This is determined by adding to nitrogen-free media the nitrogen compound to be tested.

opalescent, resembling the color of an opal.

optimum temperature, temperature at which growth is most rapid.

pellicle, bacterial growth either forming a continuous or an interrupted sheet over a fluid. peptonized, said of curds dissolved by trypsin.

persistent, lasting many weeks or months.

plumose, a fleecy or feathery growth.

pseudozoöglœæ, clumps of bacteria, not dissolving readily in water, arising from imperfect separation or more or less fusion of the components, but not having the degree of compactness and gelatinization seen in zoöglœæ.

pulvinate, in the form of a cushion, decidedly convex.

punctiform, very minute colonies, at the limit of natural vision.

raised, growth thick, with abrupt or terraced edges.

rhizoid, growth of an irregular branched' or root-like character, as in B. mycoides.

ring (same as rim), growth at the upper margin of a liquid culture, adhering more or less closely to the glass.

rapid, developing in from twentyfour to forty-eight hours. repand, wrinkled. 
saccate, liquefaction the shape of an elongated sack, tubular, cylindrical.

scum, floating islands of bacteria; an interrupted pellicle or bacterial membrane.

sporangia, cells containing endospores.

spreading, growth extending much beyond the line of inoculation, i.e. several millimeters or more. stratiform, liquefying to the walls of the tube at the top and then proceeding downwards horizontally.

transient, a few days. turbid, cloudy with flocculent particles; cloudy plus flocculence. umbonate, having a button-like, raised center.

undulate, border wavy, with shallow sinuses. vermiform-contoured, growth like a mass of worms, or intestinal coils.

verrucose, growth wart-like with wart-like prominences. villous, growth beset with hairlike extensions.

viscid, growth follows the needle when touched and withdrawn; sediment on shaking rises as a coherent swirl.

zoöglœæ, firm gelatinous masses of bacteria, one of the most typical examples of which is the Streptococcus mesenterioides of sugar vats (Leuconostoc mesenterioides), the bacterial chains being surrounded by an enormously thickened firm covering, inside of which there may be one or many groups of the bacteria. 


\section{TEXT-BOOKS ON GENERAL AND DAIRY BACTERIOLOGY}

Chester, A Manual of Determinative Bacteriology. The Macmillan Company, New York, 1901.

Coxs, Bacteria in Milk. Orange Judd Co., New York, 1907.

Fiscrer, Structure and Functions of Bacteria. Clarendon Press, New York, 1900.

Frost, Laboratory Bacteriology. The Macmillan Company, New York, 1903.

Jexsen, Essentials of Milk Hygiene. Translation by Pearson. J. B. Lippincott Company, Philadelphia, 1907.

LAFAr, Handbuch der Technischen Mykologie. Second Edition.

Gustav Fischer, Jena, 1904-1909.

Lenmaxn and Neumane, Atlas and Principles of Bacteriology.

W. B. Saunders \& Company, Philadelphia, 1901.

"Milk and its Relation to Public Health" (by various authors),

Bulletin 41, Hygienic Laboratory. United States Public Health and Marine Hospital Service, Washington, 1908.

MurR and Ritcrie, Manual of Bacteriology. Fourth Edition.

Henry Froude, and Hodder \& Stoughton, Edinburgh, 1907.

Prescott and Wixslow, Elements of Water Bacteriology. Second

Edition. Wiley \& Sons, New York, 1908.

"Report of the Cominittee on Standard Methods of Water Analysis to the Laboratory Section of the American Public Health Association." Journal of Infectious Diseases, Supplement No. I, Chicago, 1905.

Russell, Outlines of Dairy Bacteriology. Eighth Edition. H. L. Russell, Madison, Wisconsin, 1907.

Swithenbaxk and Newnan, Bacteriology of Milk. John Murray, London, 1903. 



\section{INDEX}

Abbe condenser, 39

Acid-fast bacteria, 113

Agar, 5 ; filtering of, 7, 15 ; preparation of, 15

Alcohol, test for, 79

Ammonia, test for, 71

Anaërobic bacteria, cultivation of, 82

Aniline-water gentian violet, 43

Animals, inoculation of, 114 ; postmortem examination of, 114

Antagonism, 20

Autoclave, 10

Bacillus coli communis, $18,70,77$, 97, 106

Bacillus fluorescens liquefaciens, 97, 102

Bacillus lactis acidi, 77, 97, 106

Bacillus lactis aerogenes, 77,97, 106

Bacillus lactis longi, 80

Bacillus lactis viscosus, 79

Bacillus mesentericus ruber, 74

Bacillus mesentericus vulgatus, 79

Bacteria, direct enumeration of, 122 ; effect of creaming on distribution of, 93 ; examination of living, 49; measuring of, 51 ; preliminary cultivation of, 65 ; rôle of, in cheese ripening, 104

Bacterial species, identification of, 66

Beef extract, 5, 16

Blood-counting cell, 118

Books, reference, 145

Bouillon, 12

Broth, preparation of, 12

Brownian movement, 50

Burri culture tube, 84

Butter, analysis of, 95 ; flavor of, 95 ; keeping quality of, 101
Canada balsam, 45

Capsule stain, 48

Carbohydrates, action of bacteria on, 67

Carbol-fuchsin, 43

Cheese, analysis of, 105 ; ripening of, 103 ; sampling of, 105

Chromogenesis, 74

Colonies, study of, 32

Commercial starters, 97

Cotton plugs, 9

Cover glasses, 41 ; cleaning of, 42

Cream, examination of, 94

Cultures, incubation of, 23, 24; testing purity of, 31

Diaphragm, iris, 39

Diphtheria, 111

Enzymes, 104

Fermentation, acid, 76 ; alcoholic, 78 ; bitter, 80 ; butyric, 81 ; cycle of, 80 ; sweet-curdling, 77

Fermentation tests, 107

Fibrin, staining of, 122

Fishing, 33

Flagella, staining of, 47

Fuchsin, 43

Galactase, 104

Gas, analysis of, 69 ; measurement of, 69 ; production of, 68

Gasometer, Frost's, 68

Gelatin, 5 ; effect of heat on, 14 ; filtering of, 7 ; preparation of, 14

Gentian violet, 43

Gerber fermentation test, 107

Glassware, 1 ; cleaning of, 2 ; sterilization of, 3 
Gram's stain, 43

Gram's solution, 47

Hanging block, 51

Hanging drop, 49

Heat, effect on milk, 89

Heated milk, detection of, 91

Homogeneous milk, 90

Incubation, temperature of, 23 ; time of, 23

Indol, test for, 72

Inhibition, 20

Iodine solution, 43

Iodoform, 79

Leucocytes, 116, 117

Light, 39

Litmus, 5 ; preparation of, 18

Litmus lactose agar, 29

Litmus lactose gelatin, 29

Litmus milk, 17

Lugol's solution, 43

Manure, contamination of milk from, 57

Meat infusion, 5

Media, care of, 11 ; clearing of, 16 ; filtering of, 7 ; ingredients of, 5 ; neutralization of, 6 ; reaction of, 5 ; sterility of, 11,17 ; sterilization of, 9 ; sugar-free, 18

Methylene blue, 43

Micrometer, ocular, 51 ; stage, 52

Micron, 52

Microscope, 37 ; accessories of, 37

Milk, acid fermentation of, 76 ; acidity of, 16 ; agar, 75 ; alcoholic fermentation of, 78 ; analysis of, 19,29 ; bitter fermentation of, 80 ; butyric fermentation of, 81 ; condensed, 88 ; contamination of, 53 ; cycle of fermentations in, 80 ; determination of acidity of, 76 ; digestion of, 77 ; dilution of, 21 ; examination of : for diphtheria, 112 ; for fibrin, 122 ; for leucocytes, 117,120 ; for pyogenic bacteria, 114, 124 ; for tuberculosis, 112, 114 ; for typhoid, 112 ; homogeneous, 90 ; hygiene of, 110 ; microscopical examination of, 116 ; pasteurization of, 89 ; preservation of, 86 ; ropy, 79 ; slimy, 79 ; sweet-curdling of, 77

Needles, 30 ; Ravenel's, 31 ; sterilization of, 31

Nessler solution, 71

Nitrites, test for, 71

Object slides, 41 ; cleaning of, 41

Oidium lactis, 81

Oil-immersion objective, 40

Oxygen, relation of bacteria to, 72

Pasteurization, 89

Pedesis, 50

Pepsin, 104

Peptone, 5 ; solution of, 72

Physiological salt solution, 18

Pipettes, calibration of, 125 ; preparation of, 125 ; sterilization of, 4

Plate cultures, 31 ; counting of, 22 ; incubation of, 23 ; study of, 32

Preservatives, 87

Pure cultures, isolation of, 30

Pus cells, 116

Qualitative analysis, 29

Quantitative analysis, 19, 26 ; accuracy of, 26 ; comparative method, 28 ; expression of results, 25

Rennet, 104

Ropy milk, 48

Sanitary milk, plan for preparation of, 63

Separator slime, examination of, 94

Shake cultures, 69

Spore stain, 48

Spores, test for, 73

Stab cultures, 34 ; study of, 35

Stained preparations, examination of, 46 ; making of, 44 ; mounting of, 45

Staining solutions, 42

Staphlococcus pyogenes aureus, 116 Starters, 97 ; flavor-producing properties of, 100 ; preservation of properties of, 100 ; purity of, 97 ; vitality of, 99 
Sterilization by dry heat, 3 ; by moist heat, 9

Storch's reaction, 91

Strainers, 61

Streak cultures, study of, 35

Streptococcus Hollandicus, 77

Streptococcus pyogenes, 115,116 , 124

Sugars, 5; effect of heat on, 17 ; media free from, 70

Temperature, effect of, on bacterial growth, 88 ; relation of bacteria to, 73

Test-tube cultures, 33 ; study of, 35 Thermal death point, 87
Thermometer scales, 138

Tuberculosis, 110

Tubes, plugging of, 9

Typhoid fever, 111

Udder, bacteria from, 58; infection of, 59

Utensils, contamination of milk from, 60 ; washing of, 63

Wash water, effect of, on butter, 101 ; testing of, 102

Water blanks, preparation of, 18

Wisconsin Curd Test, 107

Yeasts, isolation of, 78

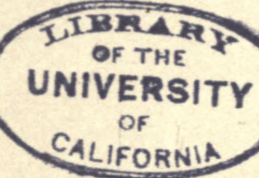






THIS BOOK IS DUE ON THE LAST DATE STAMPED BELOW

\section{AN INITIAL FINE OF 25 CENTS}

WILL BE ASSESSED FOR FAILURE TO RETURN THIS BOOK ON THE DATE DUE. THE PENALTY WILL INCREASE TO 50 CENTS ON THE FOURTH DAY AND TO \$1.00 ON THE SEVENTH DAY OVERDUE.

(BIOLOGY LIBRARY)

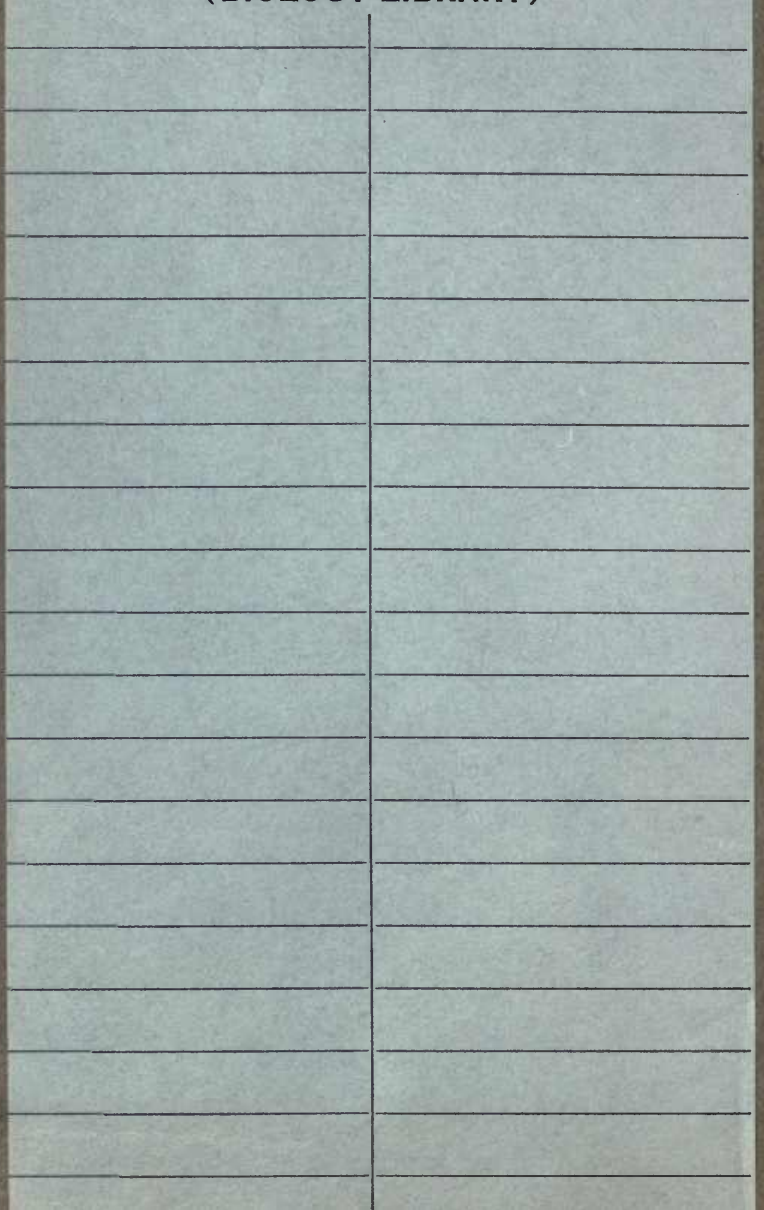




\section{QR121 R78}


